LBL-35348

\title{
Interpretation of HRTEM Images \\ by Image Simulation: \\ An Introduction to Theory and Practice
}

\author{
M.A. O'Keefe \\ Materials Sciences Division \\ National Center for Electron Microscopy \\ Lawrence Berkeley Laboratory \\ University of California, Berkeley, CA 94720
}

Notes for 1994 MSA Tutorial

Microscopy Society of America

New Orleans, LA., 7/31-8/5/94

This work was supported in part by the Director, Office of Energy Research, Office of Basic Energy Sciences, Materials Science Division of the U.S. Department of Energy under Contract No. DE-AC03-76SF00098.

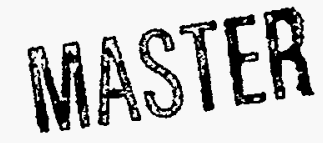




\section{DISCLAIMER}

This report was prepared as an account of work sponsored by an agency of the United States Government. Neither the United States Government nor any agency thereof, nor any of their employees, make any warranty, express or implied, or assumes any legal liability or responsibility for the accuracy, completeness, or usefulness of any information, apparatus, product, or process disclosed, or represents that its use would not infringe privately owned rights. Reference herein to any specific commercial product, process, or service by trade name, trademark, manufacturer, or otherwise does not necessarily constitute or imply its endorsement, recommendation, or favoring by the United States Government or any agency thereof. The views and opinions of authors expressed herein do not necessarily state or reflect those of the United States Government or any agency thereof. 


\section{DISCLAIMER}

Portions of this document may be illegible in electronic image products. Images are produced from the best available original document. 


\title{
Interpretation of HRTEM images
}

by image simulation:

\section{an introduction to theory and practice.}

\author{
Michael A. O'Keefe
}

National Center for Electron Microscopy

University of California, LBL, Berkeley

August 05, 1994

This work was supported by the Director, Otice of Energy Research.

Office of Basic Energy Sciences, Materials Science Division.

U.S. Department of Energy under Contract No. DE AC-08-76SF00098.

This tutorial describes the use of image simulation as an aid to interpretation of high-resolution transmission electron microscope images.

The topics include some image processing as well as image simulation.,

Image processing is the manipulation of experimental images in order to extract some desired information.

Image simulation is the generation of a computed or simulated image from a model structure. It requires a detailed knowledge of the process of image formation in the high-resolution transmission electron microscope.

This tutorial will concentrate on image simulation, with examples of image processing appearing only as required as illustrations.

Because this is an introduction, the theory of image simulation will be described, but not explored in depth.

The practice of image simulation will be covered in sufficient detail to enable the student to understand the functions of the various steps in the computations, and the parameters necessary for their evaluation. 


\section{- Outline -}

What use is HRTEM image simulation?

History - image simulation was conceived for structure determination.

Uses -- the uses of image simulation and image processing -- structure determination is only one of five uses for image simulation.

Structure determination -- recipes and examples of structure determination by matching of experimental images and simulated ones.

How does HRTEM image simulation work?

WPO -- interpreting images with the weak phase-object approximation; why do some images "look like" the structure?

Beyond WPO -- the steps involved in image simulation; modelling the high-resolution TEM in the computer; computing dynamical diffraction; computing lens effects.

Using HRTEM image simulation

Running simulations -- obtaining values of simulation parameters. Handling defects -- what additional precautions are necessary?

Why -

Histrory. HREM image simulation was invented to explain $10 \AA$-resolution images of block oxides.

Uses. Four uses for image simulation besides structure determination, or investigation, and an example of each.

Structure. Four examples of structure determination/investigation using image simulation, and two examples involving image processing, with the methodology used in each case.

Theory-

WPO. Many images "look like" the "structure". Just what is the relationship between a thin-crystal-Scherzer-defocus image and the arrangement of atoms within the specimen? The WPO approximation lets us see how such an image is built up from image components that are linearly-related to specimen structure factors -besides, WPO images can be computed veryquickly.

Beyond. WPO is fine for thin-crystal-Scherzer-defocus images, but what about images where dynamical diffraction and non-linear interferences matter? What parameters need to be known to use the full power of image simulation? What limits exist?

Using it -

Running. How do we run the programs? How do we find parameter values to input?

Defects. Since image simulation algorithms require a periodic real space, how do we handle non-periodic defects? What extra problems arise? 


\section{The lattice image that started image simulation}

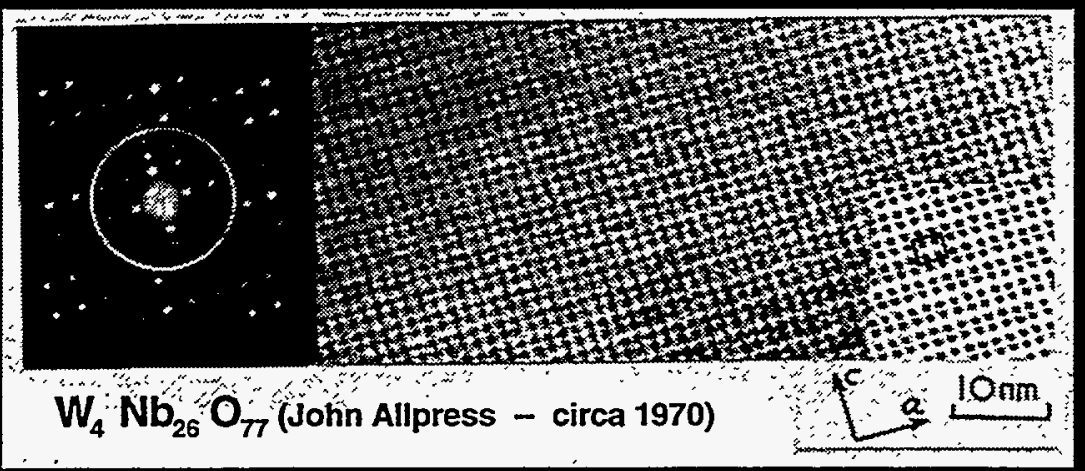

Allpress: Image at $10 \dot{A}$ resolution shows black dots arranged in a pattern corresponding to that of the tetrahedral sites in $\mathrm{W}_{4} \mathrm{Nb}_{26} \mathrm{O}_{77}$ So we must be imaging the tetrahedral sites as black dots!

Referee: Prove it!

This image, obtained by John Allpress at around $10 \AA$ resolution was the reason that a project to develop a simulation program was instituted.

The image shows that black dots appear at the correct spacings and arrangements for the positions of the tetrahedral sites, but doesn't conclusively prove that the black dots are the images of the tetrahedral sites.

When the referee insisted on proof of their interpretation, Allpress and Sanders suggested a project to develop HREM image simulation, and O'Keefe was set the task of writing code under the guidance of Moodie.

O'Keefe's simulations proved that the black dots appear at the correct positions for the tetrahedral sites, and are images of the unresolved groups of metal atoms surrounding the tetrahedral sites. 


\section{Block oxides}

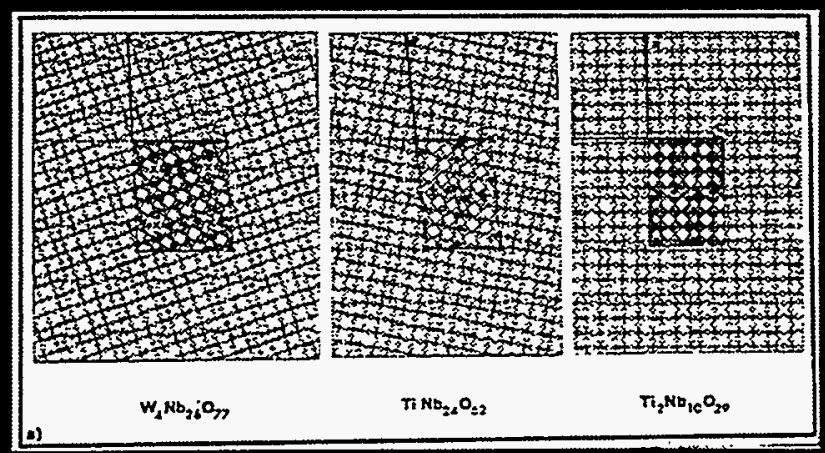

- Block oxides are built up of metal-oxygen octahedra sharing corners

- Edge-sharing octahedra create "crystallographic shear planes"

- Tetrahedral sites occur where crystallographic shear planes intersect

- Octahedra are around $3.8 \dot{A}$ across

- Spacings between tetrahedral sites are of the order of $10 \AA$

These drawings show models of block oxides typical of those Allpress was investigating. All are built up of metal-oxide octahedra with $3.8 \AA$ spacing, and have heavy tetrahedral sites spaced at more than $10 \AA$-very suitable for the microscopes of that time. 


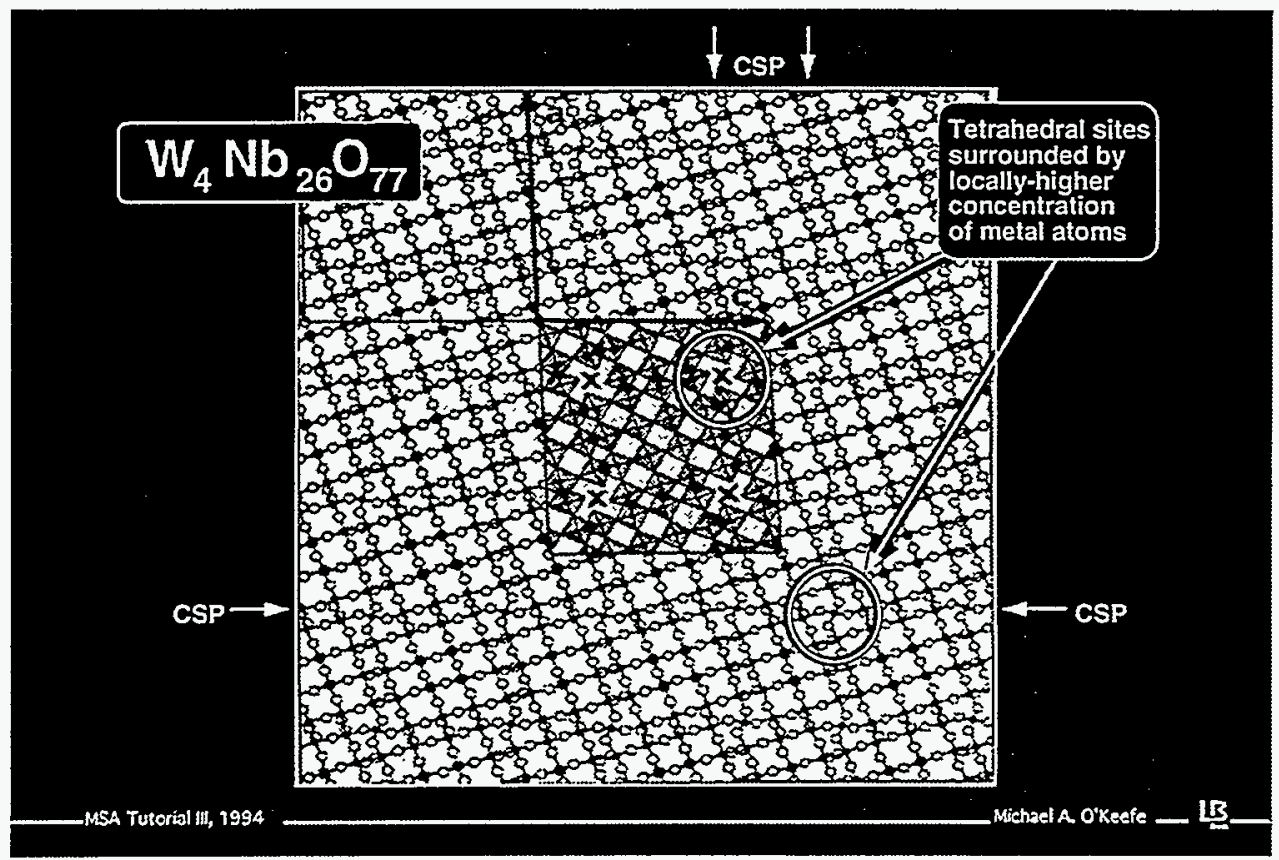

The local density of atoms is higher on the CSPS (crystallographic shear planes -- arrowed), with the highest density close to the tetrahedral sites where the CSPs intersect. This high density produces a peak in the projected potential and a black spot in the image at Scherzer defocus (at the limited resolution then in use). 


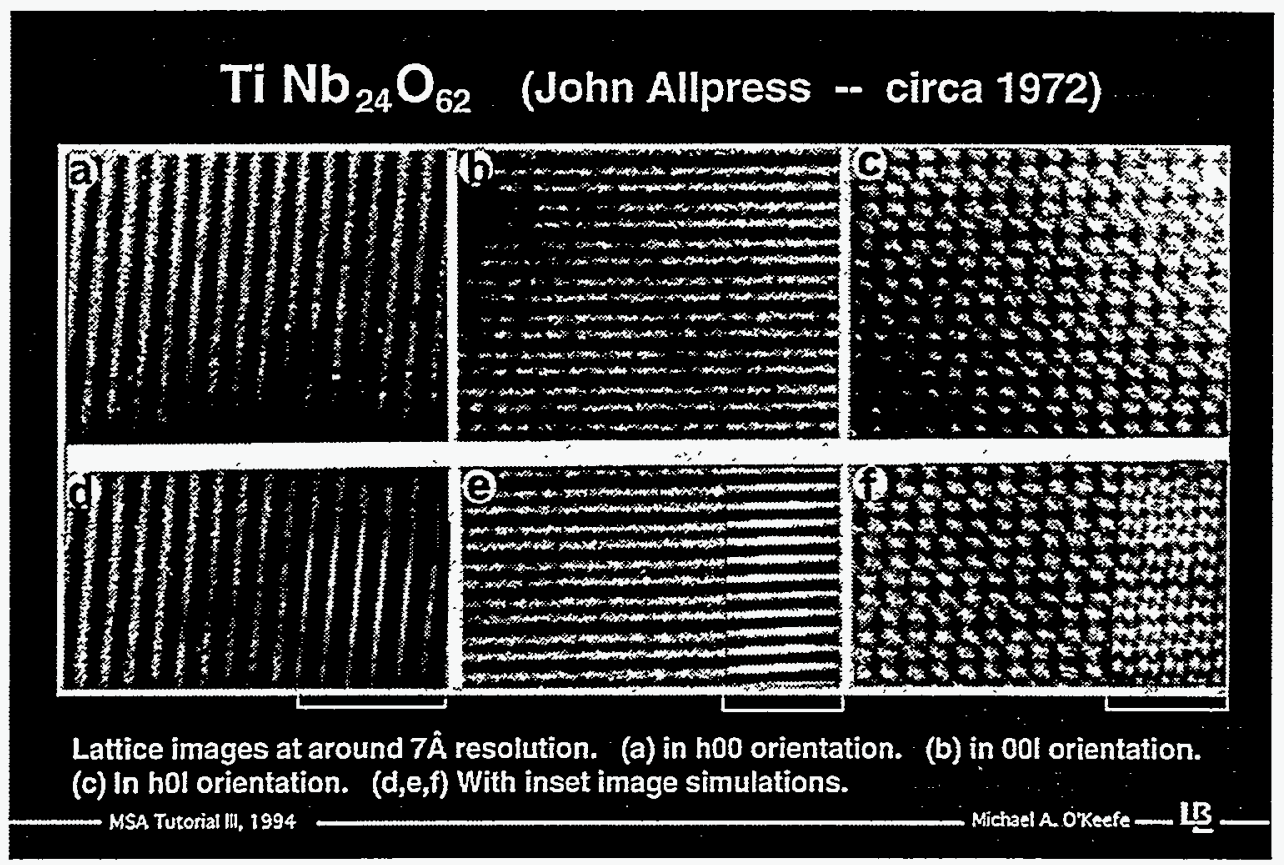

The simulations match the experimental images that were obtained in systematics orientation. The hOl simulation was computed at a resolution of around $5 \AA$, too high to match the experimental image with its resolution limited to $7 \AA \AA$. 


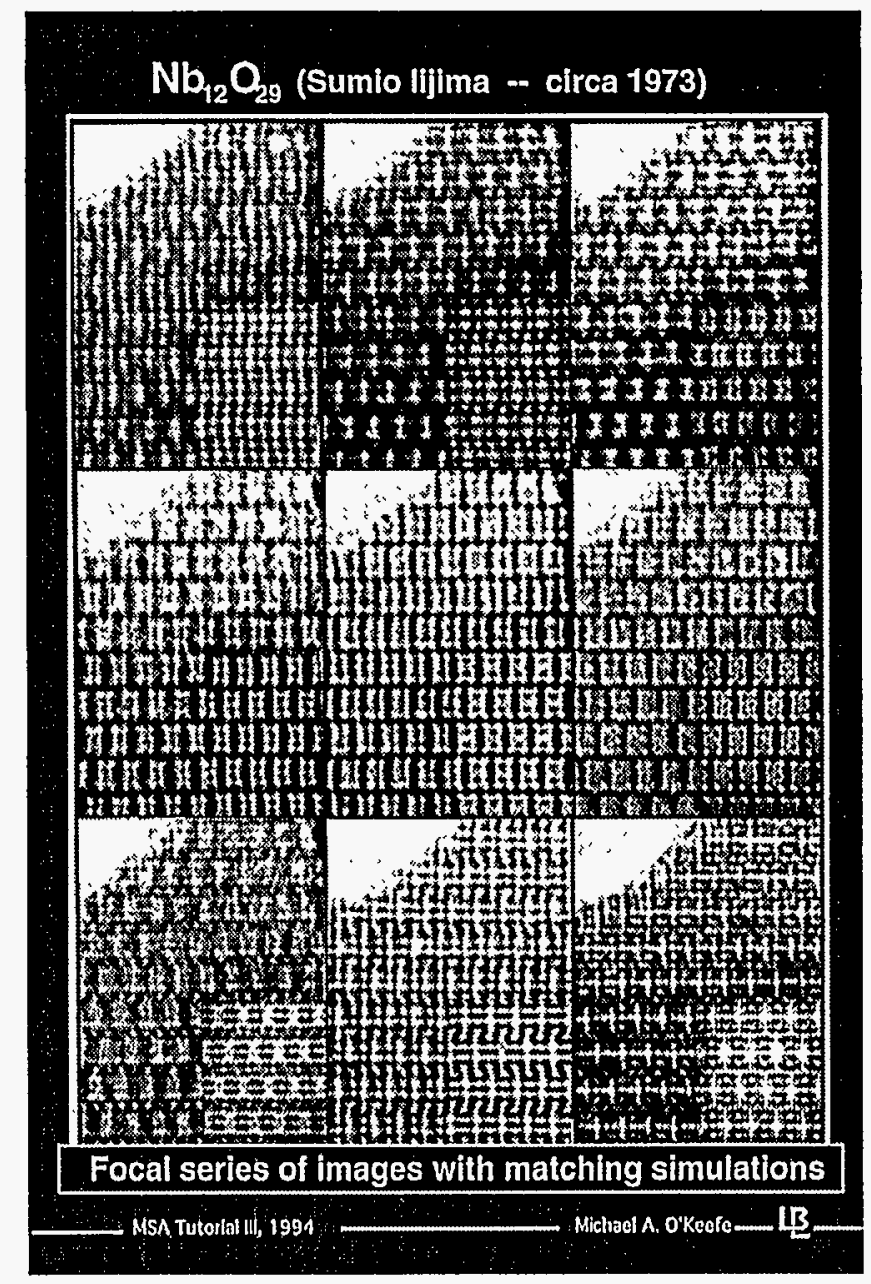

Sumio lijima obtained a focal series of images on a JEOL $100 B$ at ASU, and Michael A. O'Keefe matched them with images simulated at Melbourne University using what would later become the SHRLI simulation programs. Images were simulated for defocus values of --

\begin{tabular}{|c|c|c|}
\hline $0 \AA$ & $-160 \AA$ & $-320 \AA$ \\
\hline$-480 \AA$ & $-640 \AA$ & $-800 \AA$ \\
\hline$-960 \AA$ & $-1280 \AA$ & $-1600 \AA$ \\
\hline
\end{tabular}

with --

Cs of $1.8 \mathrm{~mm}$

crystal thickness of $35 \AA$

convergence of 1.4 milliradian

zero spread-of-focus (hadn't thought of it yet!) 


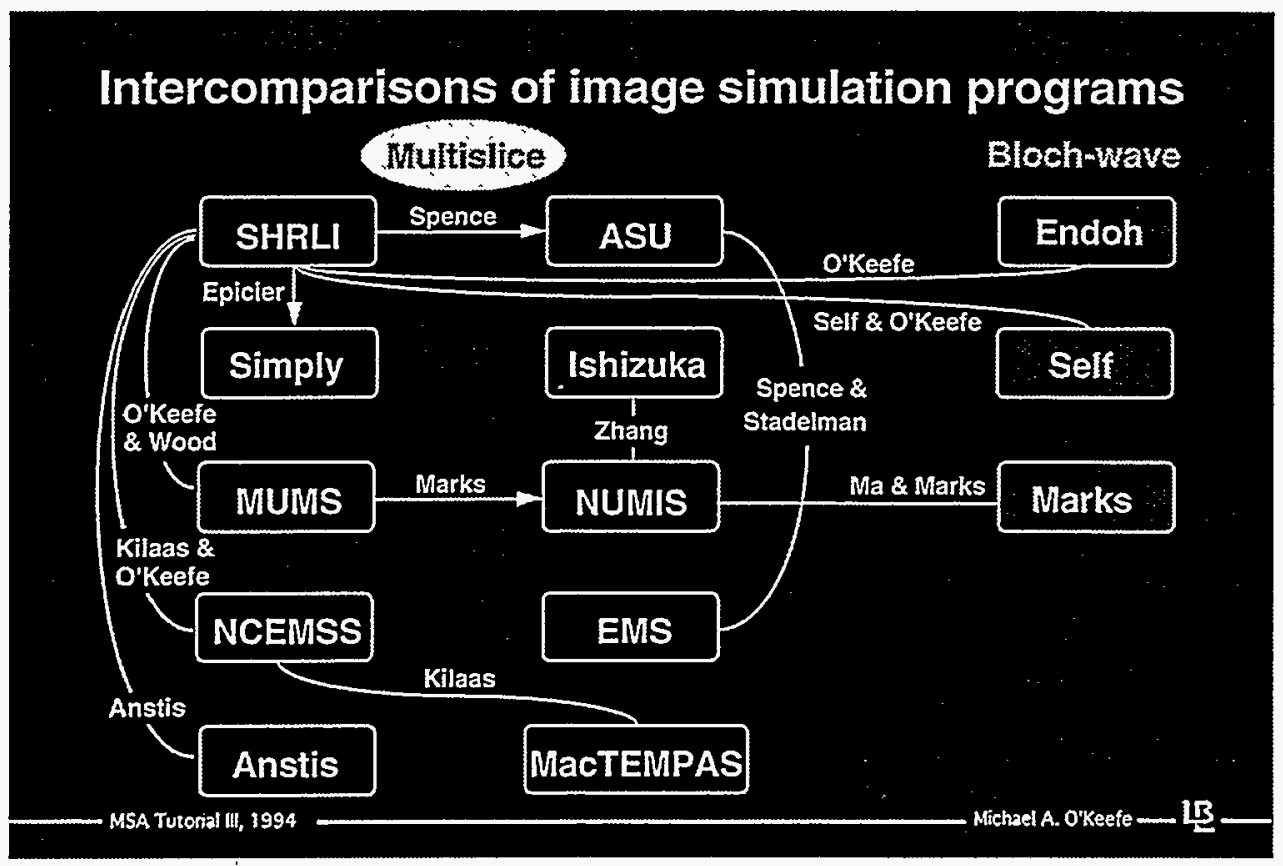

Intercomparison of simulation programs -- there are many image simulation programs available. They can be categorized as using either multislice or Bloch-wave for diffraction calculations. Results from several of these programs have been compared by various groups.

SHRLI (simulated high-resolution lattice image) was written by O'Keefe at Melbourne, ASU and Cambridge [1]. SHRLI was turned into the ASU programs, and also formed the basis for the Simply program written by Epicier[2]. The MUMS (Melboume University multislice) programs were brought to Cambridge by Wood and results compared with SHRLI. MUMS formed the basis for NUMIS. NCEMSS (the NCEM simulation system) was written by Kilaas [3] at Berkeley and results compared with SHRLI. MacTEMPAS (Mac TEM processing and simulation) was compared with NCEMSS by Kilaas. Independent multislice programs by Anstis and Ishizuka [4] have also been compared with SHRLI and NUMIS respectively. The EMS programs were written by Stadelman [5] and compared with SHRLI by Spence and Stadelman. O'Keefe compared an early version of SHRLI with a Bloch-wave program by Endoh, and Self and O'Keefe carried out a thorough comparison of SHRLI with a Bloch-wave program written by Self [6]. Ma and Marks have compared NUMIS and a Cambridge Bloch-wave program.

[1] "Computed crystal structure images for high resolution electron microscopy", M.A. O'Keefe, P.R. Buseck and S. lijima, Nature 274 (1978) 322-324.

[2] "SIMPLY: a package for the SIMulation and disPLaY of HREM images on PCs", T. Epicier and M.A. O'Keefe, 33rd Ann. Meeting of the French Electron Microsc. Soc. (SFME), Villeurbanne-Lyon, June (1993).

[3] "Interactive simulation of high-resolution electron micrographs", R. Kilaas, 45th EMSA proc. (1985) 66-69.

[4] "A new theoretical and practical approach to the multislice method", K. Ishizuka and N. Uyeda, Acta Cryst A38 (1982) $740-749$.

[5] "EMS -- a software package for electron diffraction analysis and HREM image simulation in materials science". P.A. Stadelmann, Ultramicroscopy 21 (1987) 131-146.

[6] "Practical computation of amplitudes and phases in electron diffraction", P.G. Self, M.A. O'Keefe, P.R. Buseck and A.E.C. Spargo, Ultramicroscopy 11 (1983) 35-52. 


\section{- Outline -}

What use is HRTEM image simulation?

History -- image simulation was conceived for structure determination.

Uses -- the uses of image simulation and image processing - structure determination is only one of five uses for image simulation.

Structure determination -- recipes and examples of structure determination by matching of experimental images and simulated ones.

How does HRTEM image simulation work?

WPO -- interpreting images with the weak phase-object approximation; why do some images "look like" the structure?

Bevond WPO -- the steps involved in image simulation; modelling the high-resolution TEM in the computer; computing dynamical diffraction; computing lens effects.

Using HRTEM image simulation

Bunning simulations -- obtaining values of simulation parameters. Handling defects -- what additional precautions are necessary?

- Uses. Four uses for image simulation appear in the literature (besides structure determination, or investigation).

- An example of each will be shown. 


\section{The uses of image simulation and image processing}

Image simulation -

characterize TEM

explore operating and imaging parameters "off line"

$r$ investigate improved resolution

investigate image reconstruction schemes

determine specimen structure by image matching

Image processing -

use to "clean up" experimental images for matching with simulations.

use to extract particular features from images.

\section{Image simulation examples --}

- characterizing a JEOL $100 \mathrm{~B}$ and a Hitachi $\mathrm{H}-1250$ [1]

- investigating effects of specimen and beam tilt [2]

- investigating mullite images at improved resolution [3]

- investigating a reconstruction technique [4]

- structure determination of takéuchiite [5]

- structural investigation of a Pt carbide [6]

- structural investigation of mullite [3]

- structure determination of a $\Sigma 99$ grain boundary [7]

\section{Image processing examples --}

- cleaning up yttrium di-silicate images for matching [8]

- extracting catalyst particle shapes [9]

[1] "New applications and extensions of the unique advantages of HVEM for physical and materials research". R.M. Fisher and T. Imura, Ultramicroscopy3 (1978) 3-18.

[2] "The importance of beam alignment and crystal tilt in high resolution electron microscopy", D.J. Smith, W.O. Saxton, M.A. O'Keefe, G.J. Wood and W.M. Stobbs, Ultramicroscopy11 (1983) 263-282.

[3] "Atomic Imaging of 3:2 mullite", T. Epicier, M.A. O'Keefe and G. Thomas, Acta Cryst. A46 (1990) 948-962.

[4] "Electron microscopy resolution at $1 \AA$ resolution by entropy maximization and likelihood ranking". W. Dong, T. Baird, J.R. Fryer, C.J. Gilmore, D.D. MacNicol, G. Bricogne, D.J. Smith, M.A. O'Keefe and S. Hovmöller, Nature 355 (1992) 605-609.

[5] "Electron microscopy of oxyborates. III. On the structure of Takéuchiite". Jan-Olov Bovin, M. O'Keeffe and M.A. O'Keefe, Acta Cryst. A35 (1981) 32-46.

[6] "An atomic-resolution study of a carbide phase in platinum", M.J. Witcomb, M.A. O'Keefe, C.J. Echer, C. Nelson, J.H. Turner and K.H. Westmacott, in 50th Ann Proc. EMSA, Boston, Massachusetts (1992) 20-21.

[7] "Simulated Image Maps for use in Experimental High-Resolution Electron Microscopy", Michael A. O'Keefe, Ulrich Dahmen and Crispin J.D. Hetherington, Mat. Res. Soc. Symp. Proc. 159 (1989) 453-458.

[8] " $\delta 2-Y 2 S i 2 O 7$ structure confirmed by processing and simulation of atomic-resolution images", R.S. Rai, M.A. O'Keefe and G. Thomas, Mat. Res. Soc. Symp. Proc. 139 (1988) 175-180.

[9] "Identification of periodic structure characterized from amorphous support by simulation and processing techniques", M.A. O'Keefe and M.L. Sattler, in 46th Ann Proc. EMSA, Milwaukee, Wisconsin (1988) 834-835. 


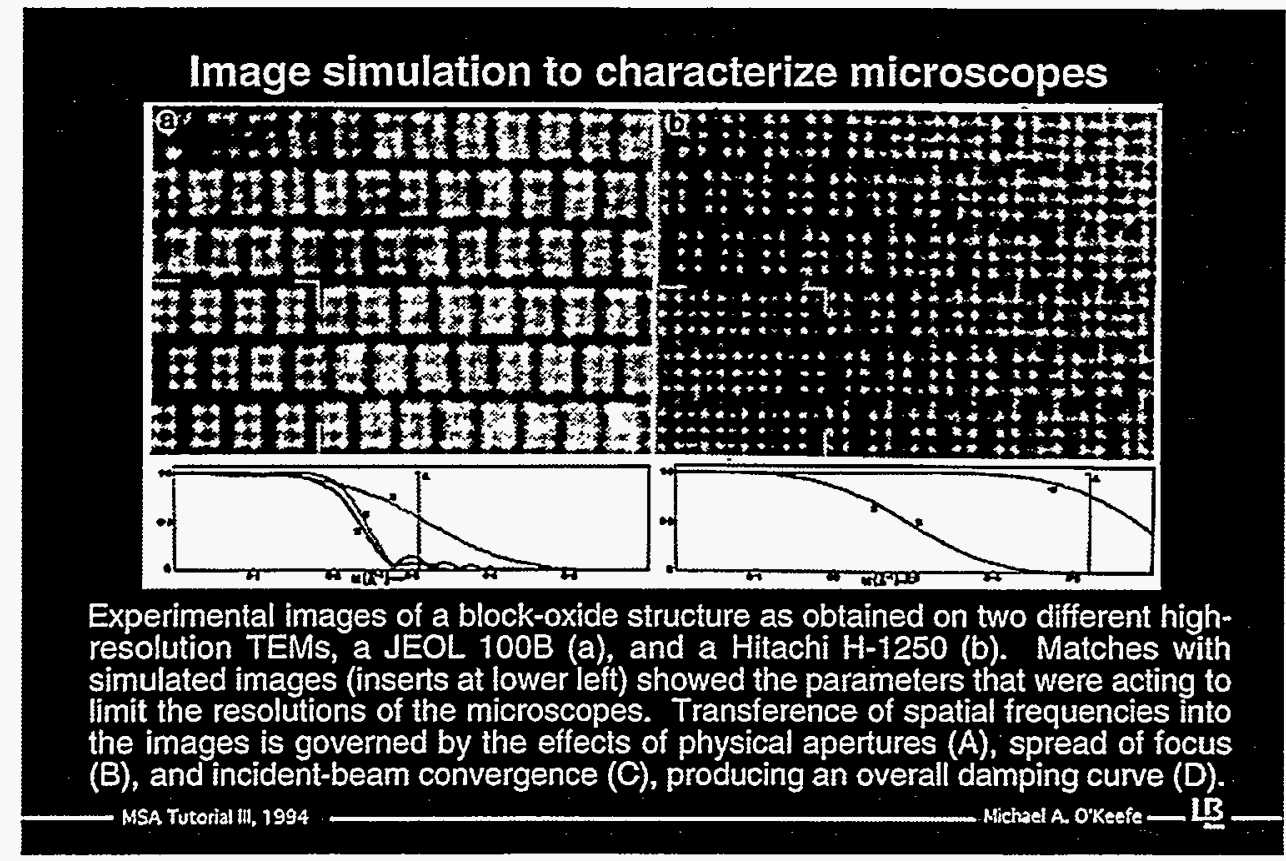

Image simulations can be used to characterize electron microscopes. The images are both "structure images" of the same titanium-niobium oxide structure -- note that there is not just one unique structure image for any particular structure.

Image (a) was taken at ASU on a JEOL $100 \mathrm{~B}$ at $100 \mathrm{keV}$ with a resolution of about $4 \AA$, and image (b) on a Hitachi $\mathrm{H}-1250$ at $1 \mathrm{MeV}$ with a resolution of about $2.5 \AA$. The inserts (lower left) are SHRLI images simulated for the conditions shown below each image.

Various effects operate to limit the microscope resolution, and the plots below each image show them as damping functions operating on the higher spatial frequencies being transferred to the image intensity spectra. The plots cover the spatial frequency range from zero to $0.6 \AA$.

- At $100 \mathrm{keV}$ (a) the physical aperture (A) limits resolution to $3.2 \AA$, and the effects of spread of focus $(B)$ to $2.4 \AA$, but convergence $(C)$ is the factor that determines the overall image resolution of $4 \AA$.

- At $1 \mathrm{Mev}(\mathrm{b})$ the physical aperture $(\mathrm{A})$ limits resolution to $1.9 \AA$, and the effects of convergence (C) to $1.5 \AA$, but spread of focus $(B)$ is the factor that determines the overall image resolution of approximately $2.5 \AA$.

Note: the advantages of higher resolution enjoyed by higher-voltage microscopes is somewhat offset by the effects of electron beam damage.

"New applications and extensions of the unique advantages of HVEM for physical and materials research". R.M. Fisher and T. Imura, Ultramicroscopy3 (1978) 3-18. 


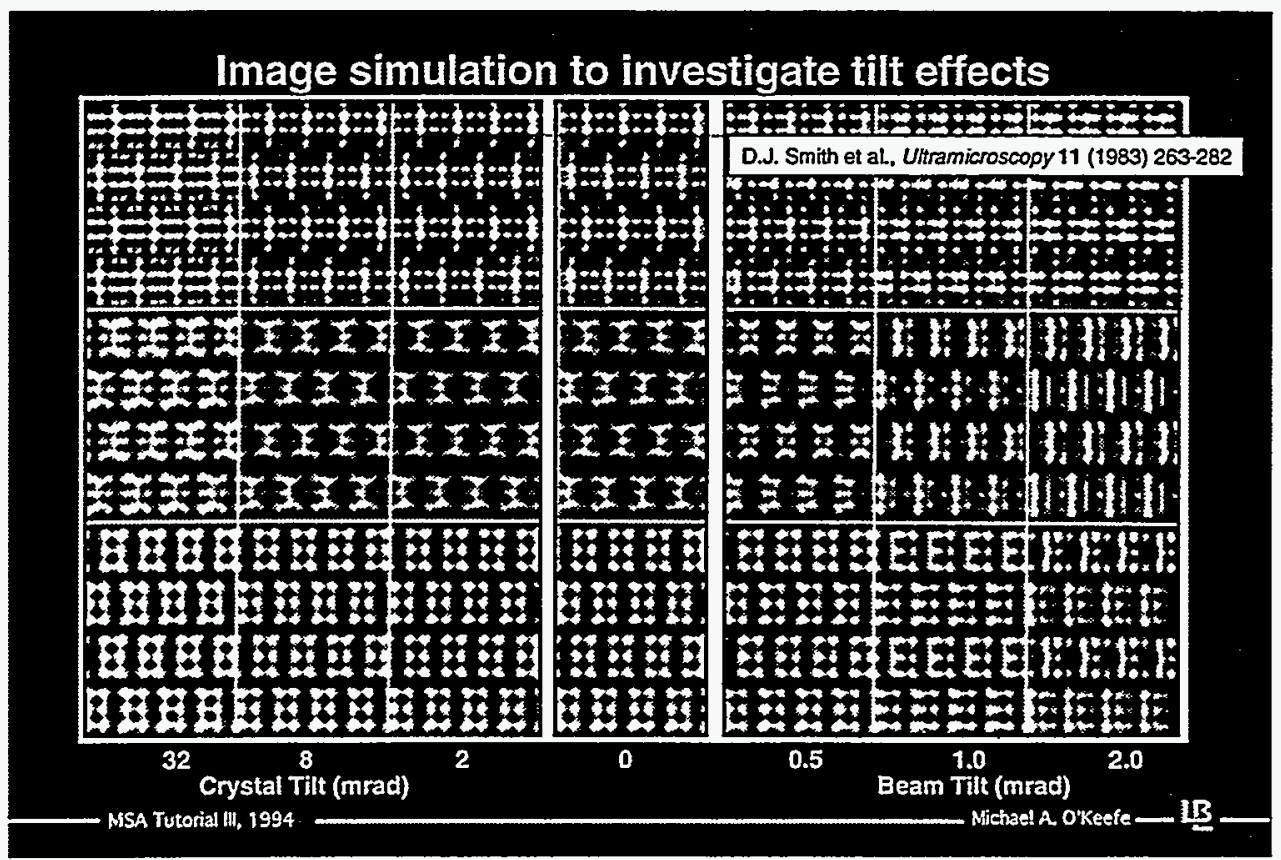

Image simulations can be used to investigate systematically the effects of various imaging parameters. These simulated images illustrate the effects of crystal tilt (out to 32 milliradian) and microscope misalignment ("beam tilt" -- out to 2 milliradian) on the same titanium-niobium oxide structure, imaged at three defocus values.

Note that modest amounts of misalignment produce much more serious image degradation than do much larger amounts of crystal tilt when the specimen is thin.

"The importance of beam alignment and crystal tilt in high resolution electron microscopy", D.J. Smith, W.O. Saxton, M.A. O'Keefe, G.J. Wood and W.M. Stobbs, Ultramicroscopy 11 (1983) 263-282. 


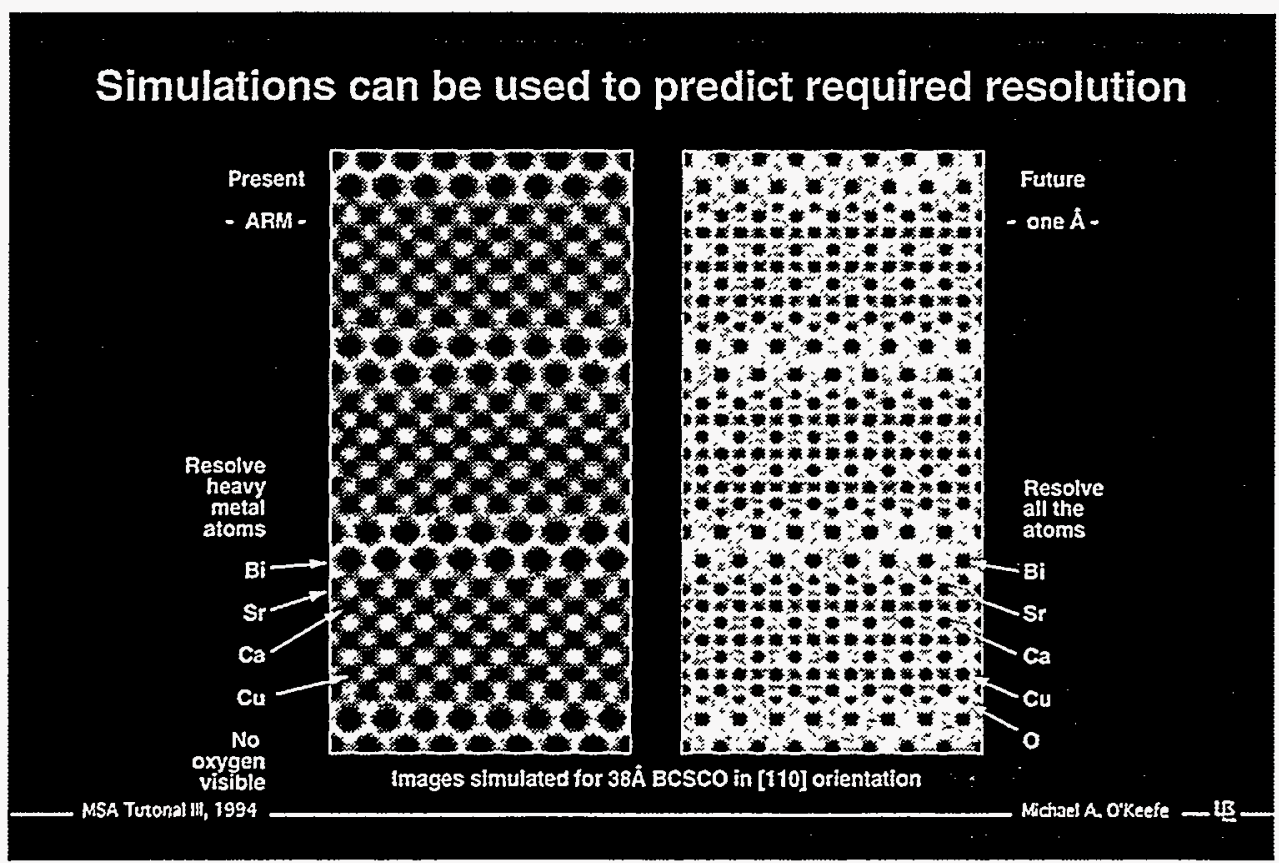

An example of the improvement in resolution that can be expected for a high-temperature super-conductor image if microscope resolution were to be improved to $1 \AA$. At the ARM resolution of $1.6 \AA$, all metal atoms can be seen. At a postulated resolution of $1 \AA$ the metal atom positions are seen more clearly and oxygens now become visible. 


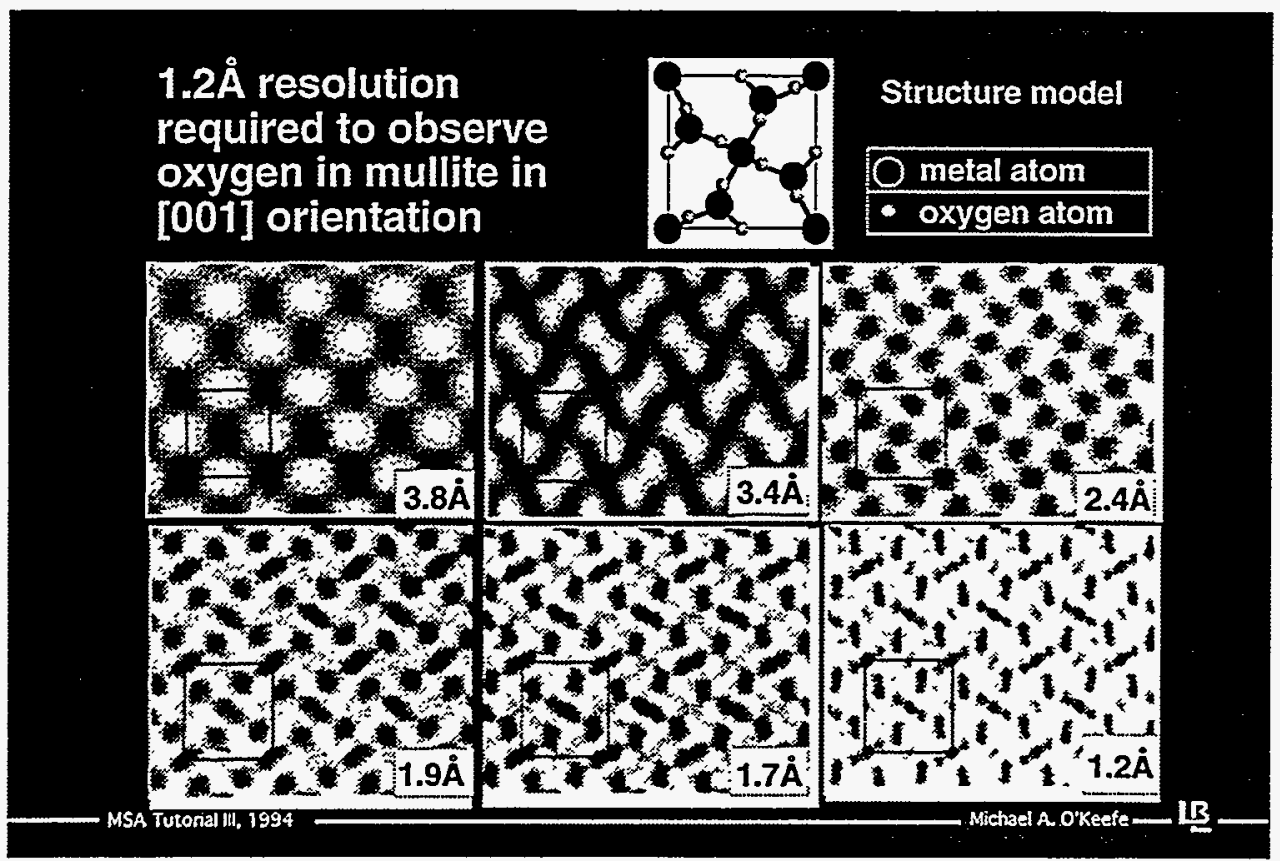

Examine improved resolution -- Mullite is an important hightemperature industrial ceramic. Its properties are governed by oxygen vacancies. A WPO-resolution series computed for the perfect structure shows that $1.2 \AA$ resolution would be required to see the oxygens, although metal atoms are clearly resolved at 2.4A. Note the misleading non-structural detail in the image at $1.7 \AA$ resolution, in which contrast at the position of the central O-metal-O triplet seems to indicate that there are two instead of three atoms.

WPO $=$ weak phase object (an image from a thin crystal, usually at Scherzer defocus) 


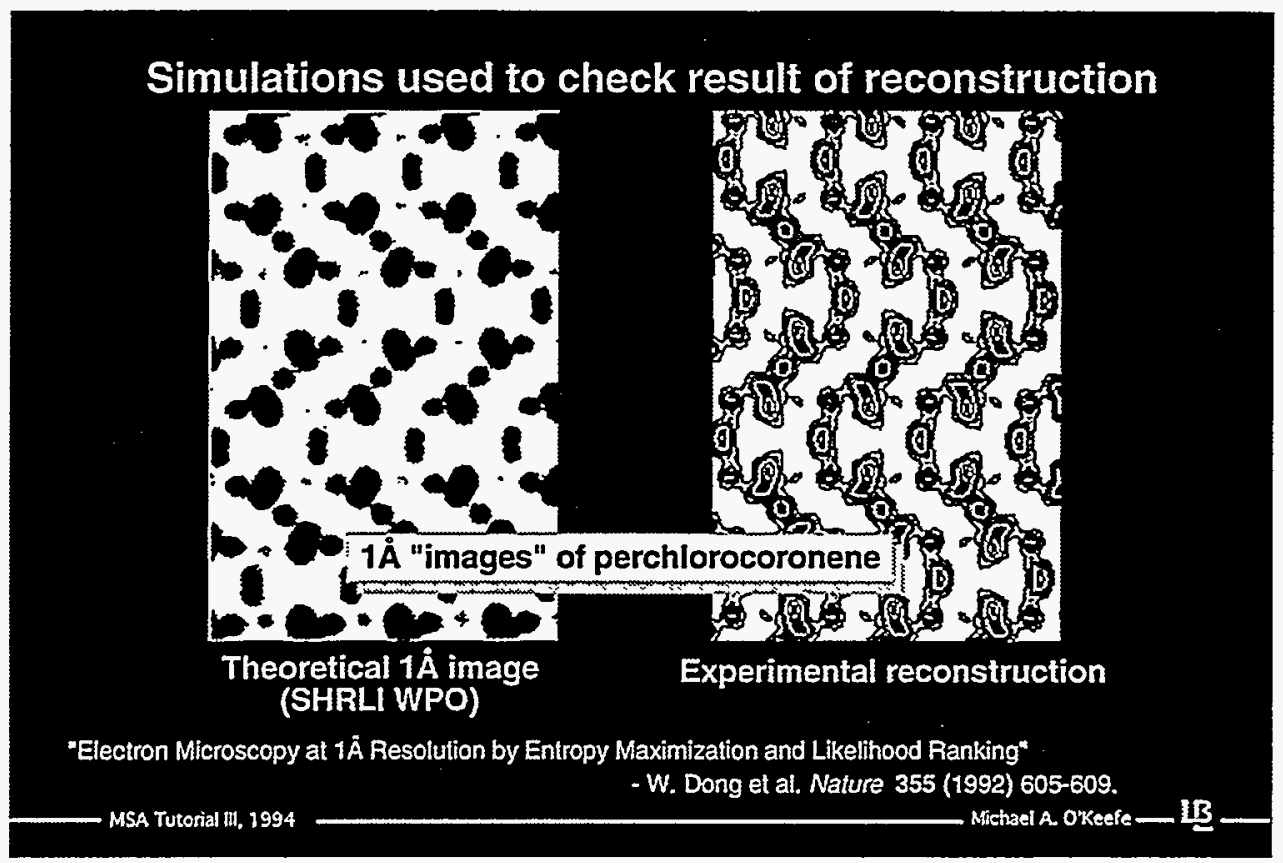

Image simulations have been used to check the validity of image reconstruction techniques. Here an "image" of perchlorocoronene (right) has been generated by combining an experimental TEM image with electron diffraction data out to $1 \AA$ resolution. $A S H R L I$ image computed at a resolution of $1 \AA$ confirms the correctness of the reconstruction method and shows that individual chlorine and carbon atoms are truely resolved.

"Electron microscopy resolution at $1 \AA$ resolution by entropy maximization and likelihood ranking". W. Dong, T. Baird, J.R. Fryer, C.J. Gilmore, D.D. MacNicol, G. Bricogne, D.J. Smith, M.A. O'Keefe and S. Hovmöller, Nature 355 (1992) 605-609. 


\section{- Outline -}

What use is HRTEM image simulation?

History - image simulation was conceived for structure determination. Uses - the uses of image simulation and image processing -- structure determination is only one of five uses for image simulation.

Structure determination -- recipes and examples of structure determination by matching of experimental images and simulated ones.

How does HRTEM image simulation work?

WPO -- interpreting images with the weak phase-object approximation; why do some images "look like" the structure?

Beyond WPO -- the steps involved in image simulation; modelling the high-resolution TEM in the computer; computing dynamical diffraction; computing lens effects.

Using HRTEM image simulation

Ruaning simulations -- obtaining values of simulation parameters. Handling defects -- what additional precautions are necessary?

\section{Why -}

- Structure Determination. The most wide-spread use of image simulation is to check the validity of structure models of perfect crystals or defects.

- Four examples of structure determination/ investigation using image simulation will be shown

- Also two examples involving image processing, with the methodology used in each case. 


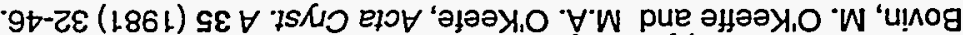
nolo-uer "әң!!

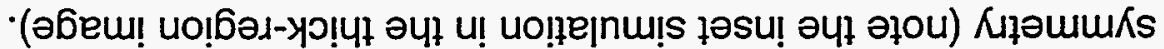

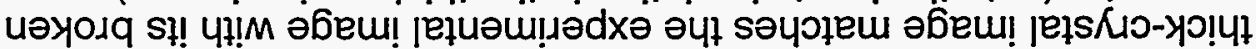

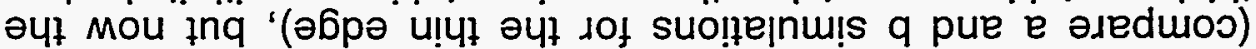

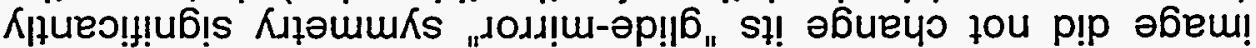

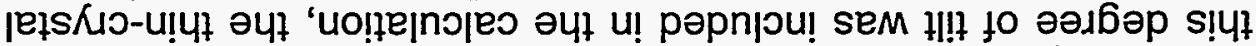

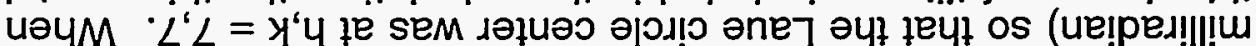

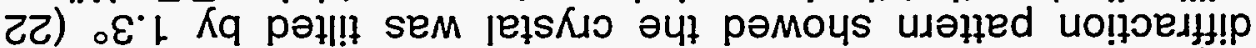

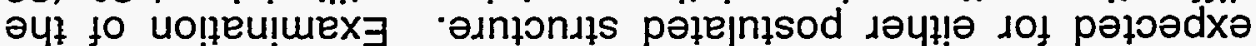

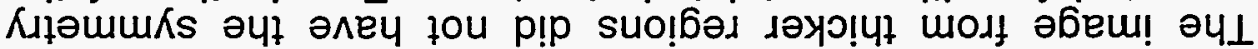

'nS $\forall$ te suresbosd uolfejnu!s

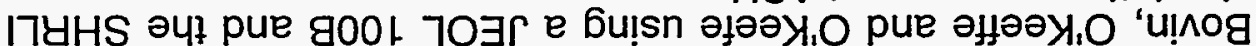

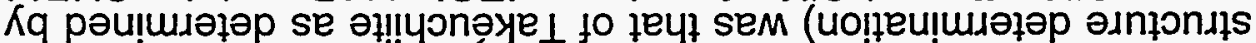

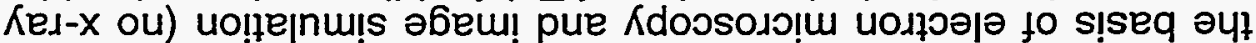

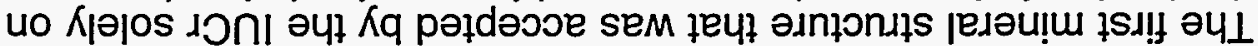

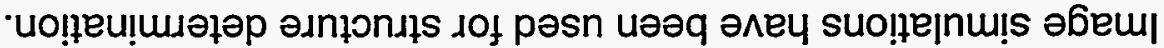

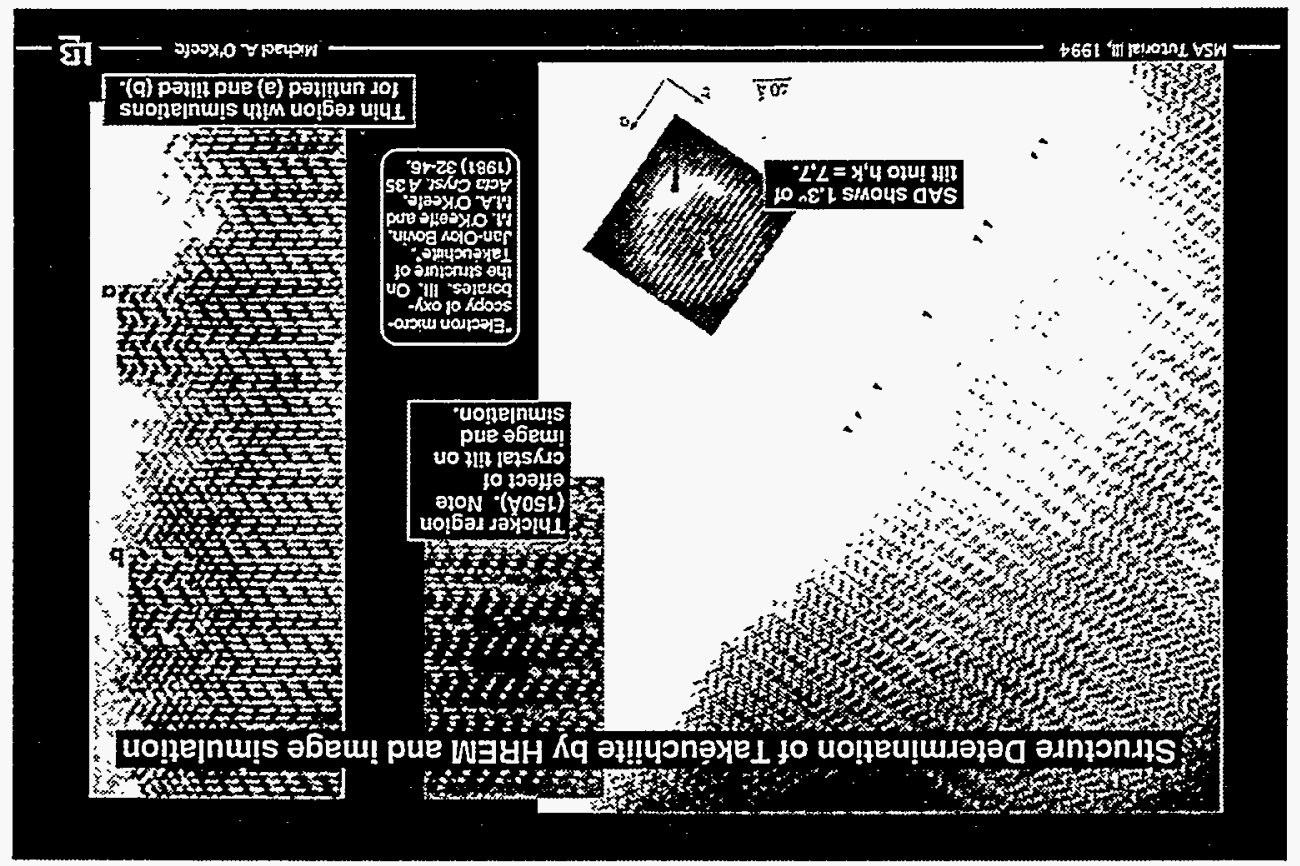




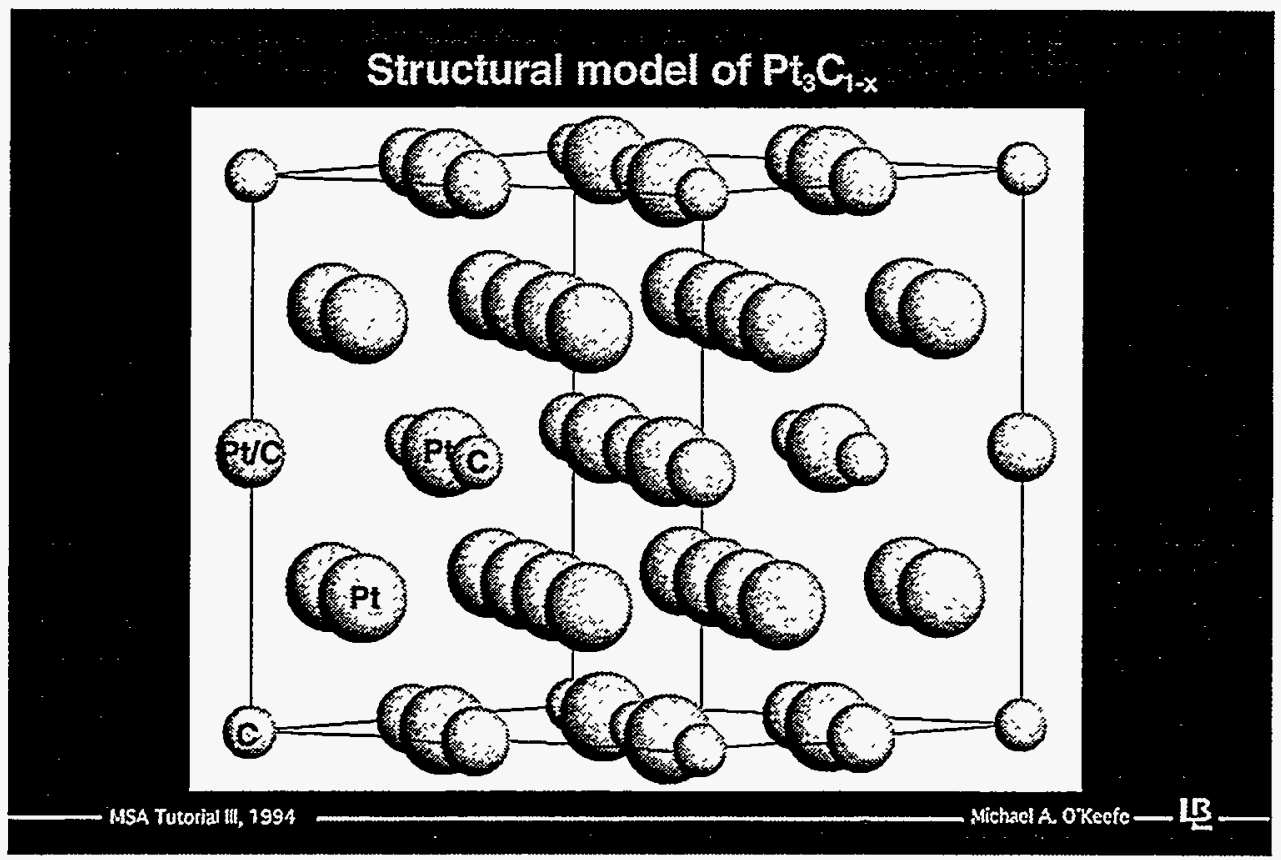

Structure determination -- Various models of a platinum- carbon structure were tested, but only one matched experimental images. The matching model required partial occupancy (shown as $\mathrm{Pt} / \mathrm{C}$ ) of some sites in order to attain the correct ratio of $\mathrm{Pt}$ to $\mathrm{C}$.

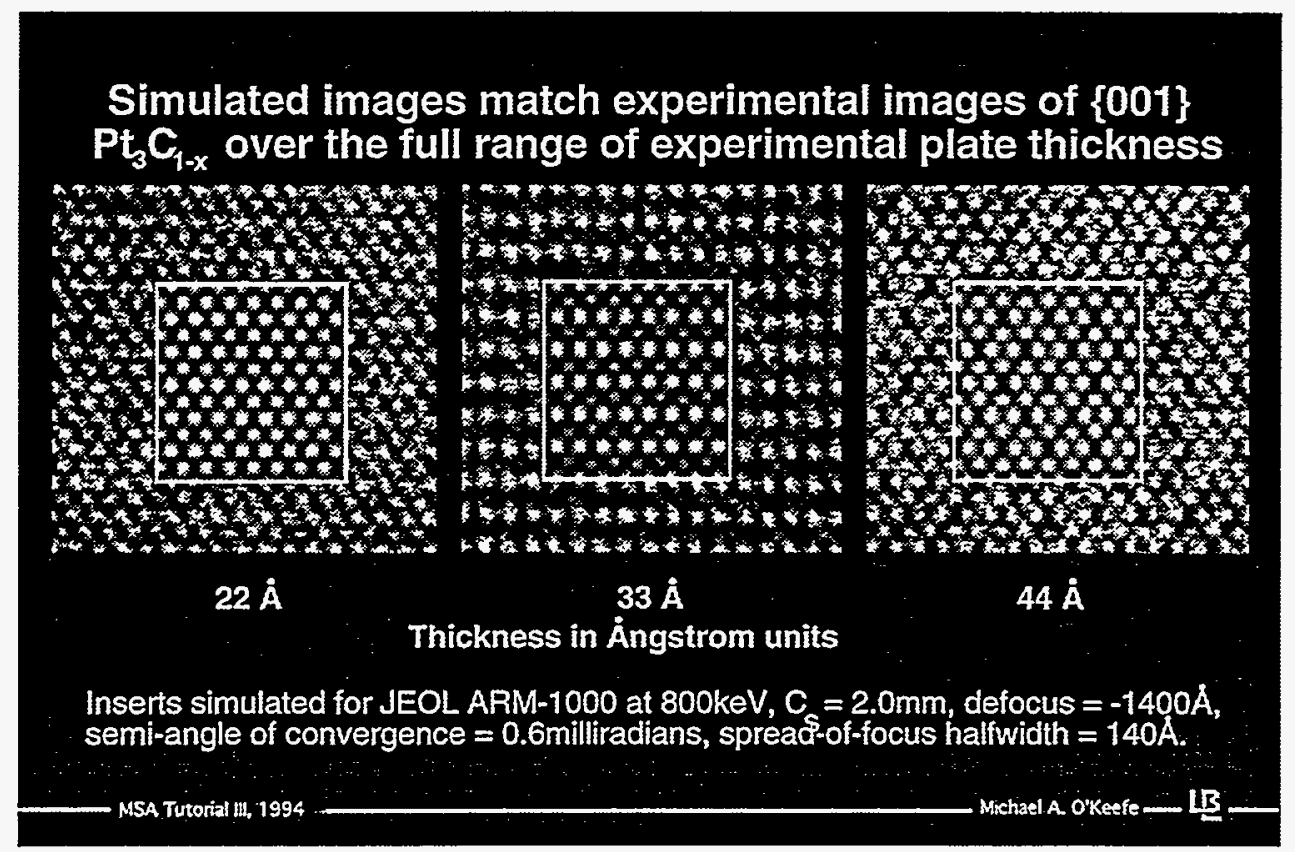

Structure determination -- Images simulated from the model with partial occupancy match experimental images as crystal thickness is changed from $22 \AA$ through $44 \AA$. Note how rapidly the image changes with change in thickness. Images are far from WPO conditions because of the high scattering density of the structure (lots of heavy platinum).

"An atomic-resolution study of a carbide phase in platinum", M.J. Witcomb, M.A. O'Keefe, C.J. Echer, C. Nelson, J.H. Turner and K.H. Westmacott, in 50th Ann Proc. EMSA, Boston, Massachusetts(1992) 20-21. 


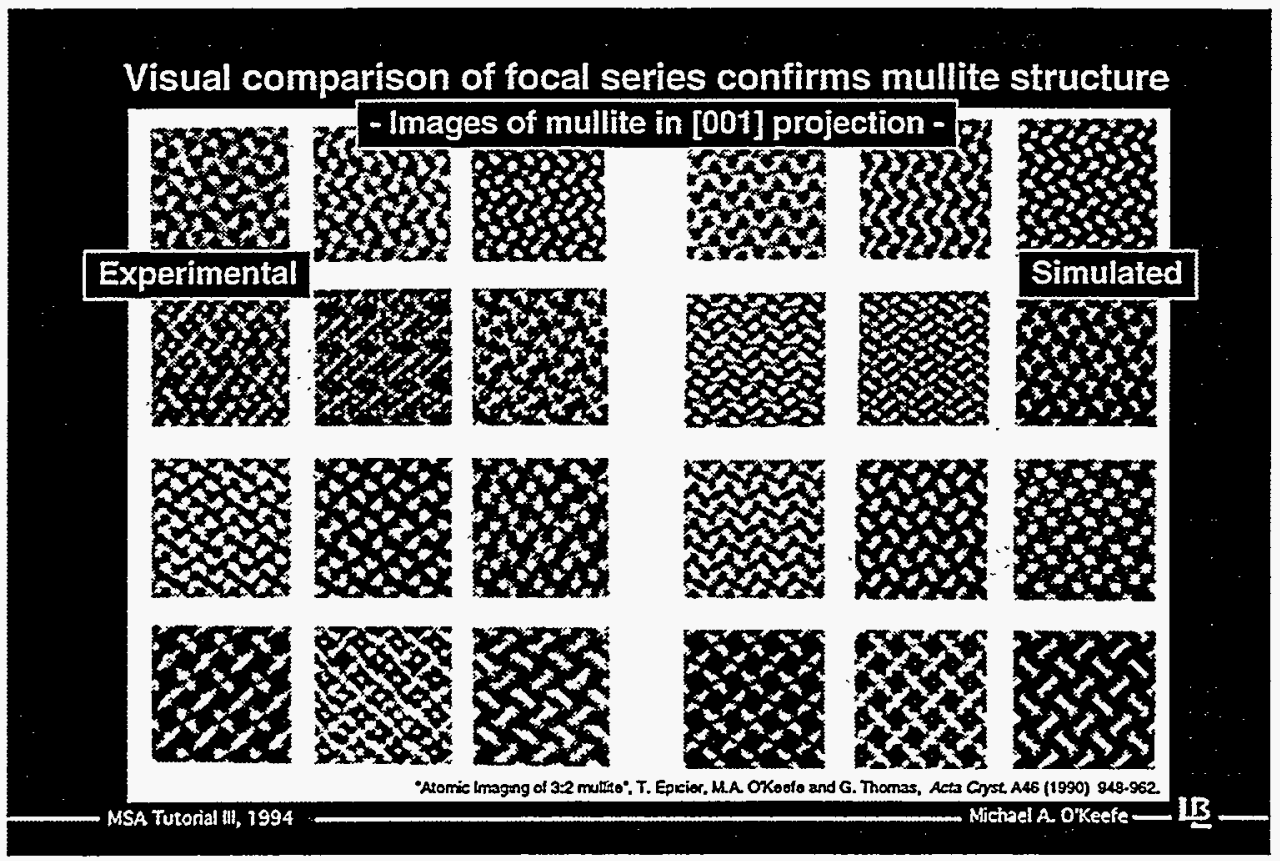

Comparison of a focal series of simulated (left) and experimental (right) images of mullite taken on the JEOL ARM-1000 at $800 \mathrm{keV}$.

Matching of a focal series tests the model structure with many different combinations of diffracted beams interfering over a wide variation of phases, and is a much better test of the accuracy of the model structure (and of the accuracy of the microscope parameters) than matching of just a single image.

Simulations are for a specimen thickness of $45 \AA$ and the twelve defocus values -

\begin{tabular}{|c|c|c|}
\hline$+700 \AA$ & $+450 \AA$ & $+300 \AA$ \\
\hline$+100 \AA$ & $0 \AA$ & $-200 \AA$ \\
\hline$-400 \AA$ & $-600 \AA$ & $-900 \AA$ \\
\hline$-1000 \AA$ & $-1100 \AA$ & $-1400 \AA$ \\
\hline
\end{tabular}

"Atomic Imaging of 3:2 mullite", T. Epicier, M.A. O'Keefe and G. Thomas, Acta Cryst. A46 (1990) 948-962. 


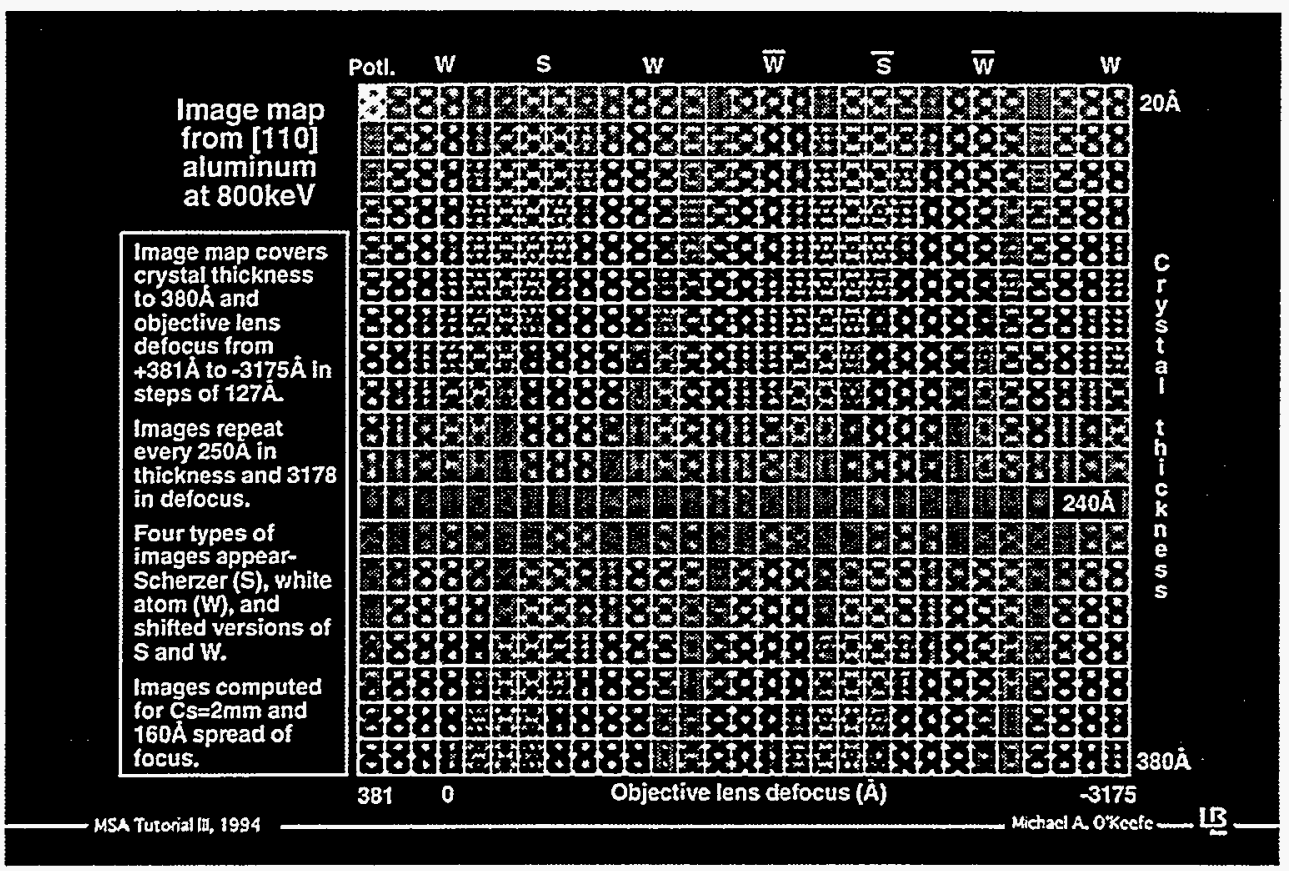

Image maps provide a way to determine the best conditions for imaging a particular structure (at least simple ones).

This map covers a full Fourier-image period in defocus $(3178 \AA$ at $800 \mathrm{keV}$ for $\mathrm{Al}$ in [110] projection). The map shows that, as well as repeating with a defocus period of $3178 \AA$, images also repeat with a thickness period of $250 \AA$; the exinction length is easily seen.

Even in this simple structure, there are many image types. And of these, there are four high-contrast ones that will tend to be chosen by the operator. In the thin-crystal area (top of figure), the Scherzer-type image (S) occurs at $-500 \AA$ with black spots at the atom positions (the projected potential is shown at top left). A reverse-contrast (white atom) image occurs at $-900 \AA$ defocus and zero defocus (and again one Fourier-image period away at $-3178 \AA$ ).

In addition, shifted "black-atom" and "white-atom" images occur at $-1400 \AA,-1950 \AA$ and $-2500 \AA$. These images, in which the black or white spots occur off the atom positions, would be dangerous ones to rely on for any structure determination of defects in this material.

"Simulated Image Maps for use in Experimental High-Resolution Electron Microscopy", Michael A. O'Keefe, Ulrich Dahmen and Crispin J.D. Hetherington, Mat. Res. Soc. Symp. Proc. 159 (1989) 453-458. 


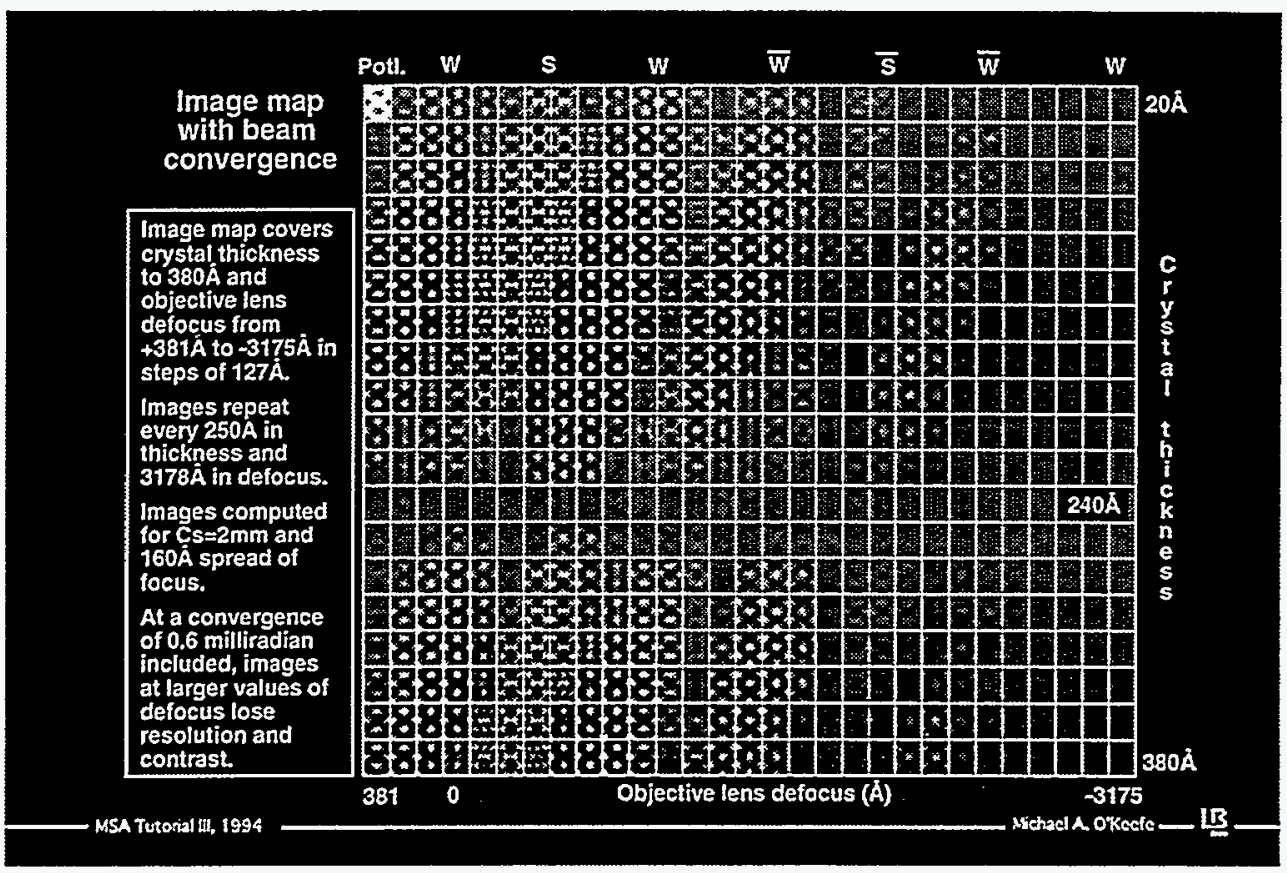

When incident beam convergence is included in the simulation, images at large defocus values lose resolution and contrast, eliminating them as useful images. But some "false" "shifted-atom" images continue to survive -- at defocus values as close to the normal operating region as $-1400 \AA$.

"Simulated Image Maps for use in Experimental High-Resolution Electron Microscopy", Michael A. O'Keefe, Ulrich Dahmen and Crispin J.D. Hetherington, Mat Res. Soc. Symp. Proc. 159 (1989) 453-458. 


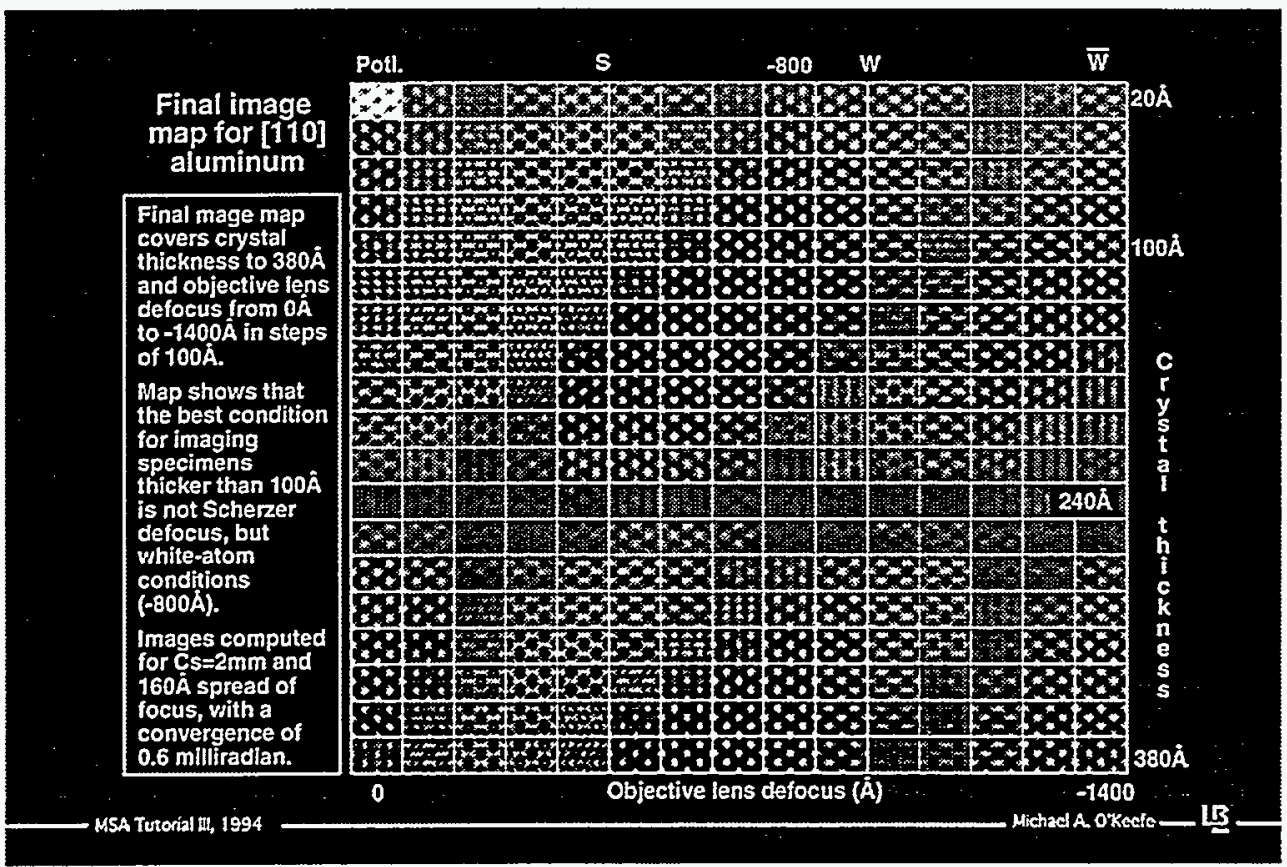

The final map covers the useful range of experimental images of $\{110\}$ aluminum for this particular high-resolution electron microscope (JEOL ARM-1000 operating at $800 \mathrm{keV}$ ).

To obtain an experimental image of a grain boundary required a specimen with a thickness of at least $100 \AA$. At $100 \AA$ thickness, the map shows that the Scherzer image is not useful, since contrast dies out at a thickness of $80 \AA$ to $100 \AA$. Instead, a defocus near to $-800 \AA$ can be used to deliver a "white-atom" image since this region of the map extends to almost $200 \AA \AA$ thickness.

Note how each "zone" of useful (high-contrast) images occupies a diagonal area in this thickness-defocus map. This "diagonalization" happens because of phase shifts that occur when dynamical scattering advances the phases of exit-surface beams by a value of more than the $\pi / 2$ predicted by WPO theory.

"Simulated Image Maps for use in Experimental High-Resolution Electron Microscopy", Michael A. O'Keefe, Ulrich Dahmen and Crispin J.D. Hetherington, Mat. Res. Soc. Symp. Proc. 159 (1989) 453-458. 


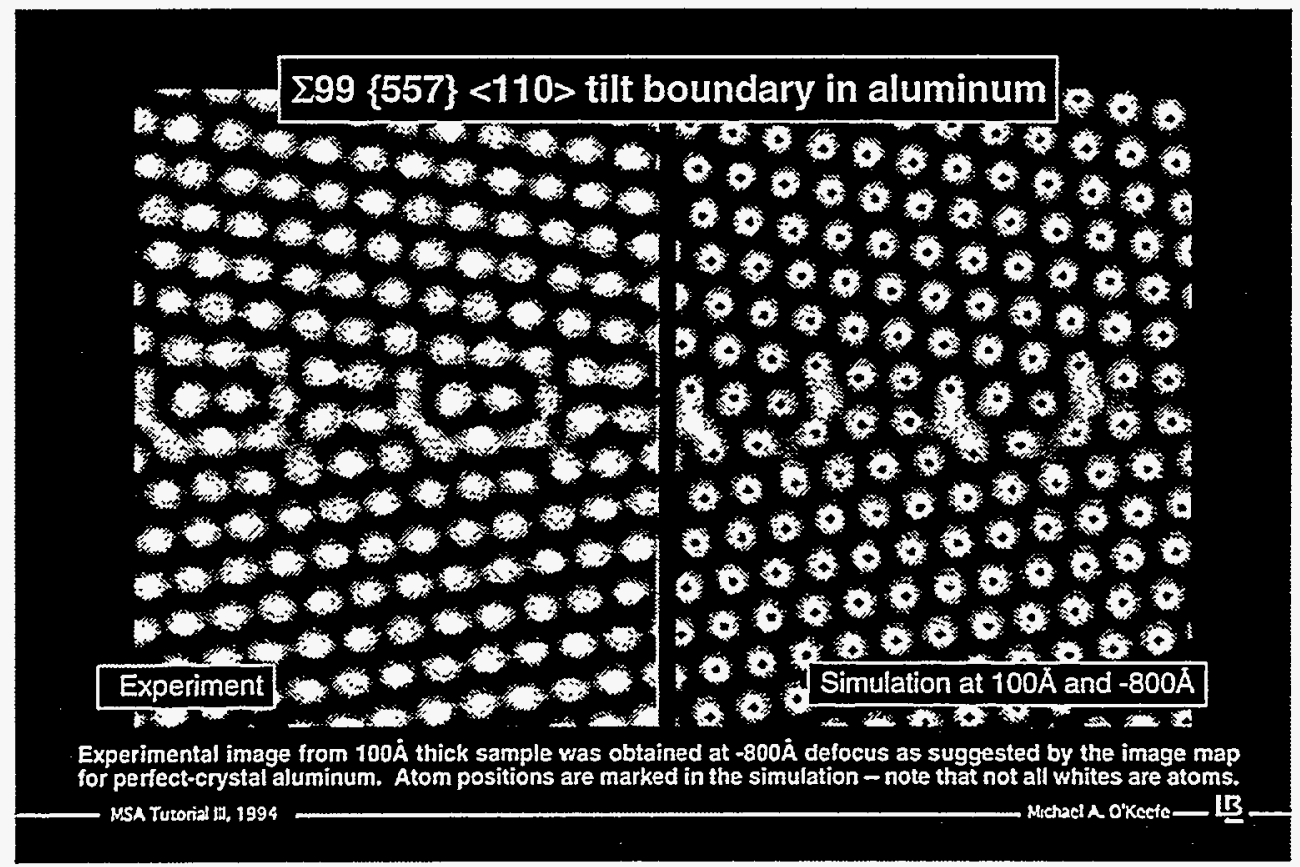

An experimental image obtained from a grain boundary at $-800 \AA$ defocus shows "white-atom" contrast (left) and matches an image simulated at $-800 \AA$ defocus and $100 \AA$ thickness (right). Atom positions in the simulation are marked with black dots.

The image map for perfect aluminum predicted good "white-atom" imaging conditions at $100 \AA$ thickness and $-800 \AA$ defocus for the perfect lattice on each side of the boundary. However, this prediction (although very useful) is only for the perfect structure. Not every white spot at the boundary corresponds to an atom position; the defect makes its own rules, and a careful image-simulation study of the full defect structure must be carried out to avoid errors.

The image area shown covers two repeats ("unit cells") along the boundary (horizontal on the page), but only one quarter of the cell used in the perpendicular direction (vertical). Because any image simulation will be "periodic" and will produce additional defects at the unit cell period in all directions due to the "periodic continuation" property of Fourier transforms, the "unit cell" used for the defect simulation must be large in the dimension perpendicular to the defect (the non-periodic direction) in order to prevent "contamination" of the defect under study by adjacent "ghost" defects.

"Simulated Image Maps for use in Experimental High-Resolution Electron Microscopy", Michael A. O'Keefe, Ulrich Dahmen and Crispin J.D. Hetherington, Mat. Res. Soc. Symp. Proc. 159 (1989) $453-458$. 


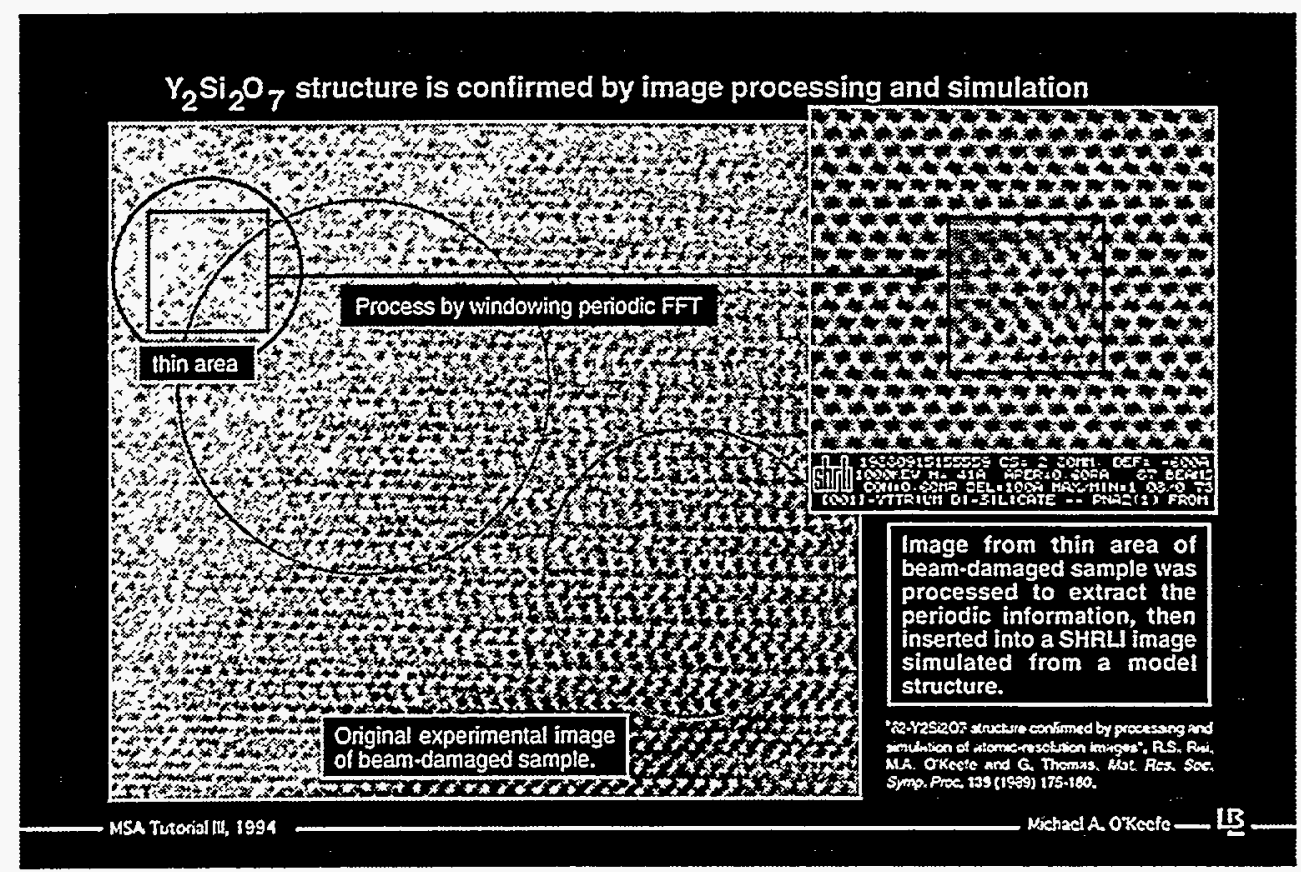

Image processing can be used to prepare an experimental image for comparison with a simulated image (and to thus confirm a postulated structure) when direct comparison of the experimental image with a simulation would be futile. In this case the experimental sample beamdamaged quite severely (and was also tilted off-axis), so that the experimental image was very "noisy".

The periodic information in the image was extracted by FFTing to obtain the image intensity spectrum, windowing the spectrum to retain only the sharp spots, then IFTing to form the new (periodic) image [1]. For comparison, the processed image was then inserted into one simulated from a postulated model.

The best match was from an area of the image coming from a thin part of the sample. Thicker parts of the sample (lower right in the experimental image) showed the wrong symmetry due to crystal tilt.

[1] " $\$ 2-Y 2 S i 2 O 7$ structure confirmed by processing and simulation of atomic-resolution images", R.S. Rai, M.A. O'Keefe and G. Thomas, Mat. Res. Soc. Symp. Proc. 139 (1989) 175-180. 


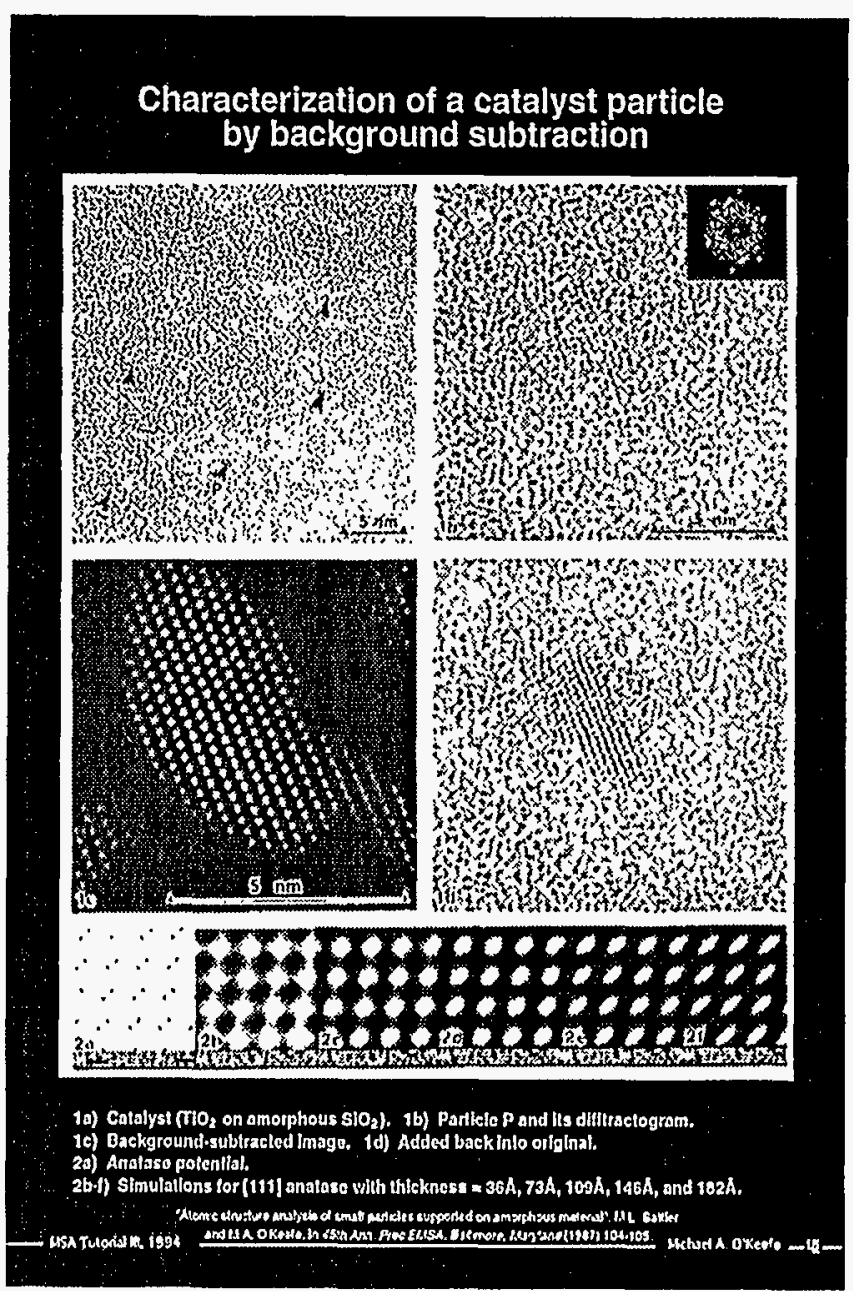

Pre-processing before comparison with simulation -. "background subtraction" for small particles.

Images were taken of a catalyst consisting of titanium dioxide crystals on amorphous silica. The problem was to characterize the crystals -- to determine their phase (rutile or anatase), shapes, sizes (including thicknesses) and orientations.

(1a) The crystalline particles are difficult to see due to the amorphous support, but the one marked $P$ was selected and is shown at twice the magnification in (1b). The image was FFTed, producing the diffractogram shown as an insert. The method of "background subtraction" was applied in order to remove the amorphous contribution to the Image; the result is shown in (1c) at twice the magnification. Addition of the crystal image to the original enhances its contrast and shows it in context (1d).

Examination of the crystal Image (and the diffractogram) shows that the particle is anatase in [111] orientation. Simulations of anatase images match the crystal image (1c), and show that the white spots in the image correspond to the tunnels in the projected potential (fig 2a). A thickness series (fig $2 b-f$ ) shows that the white spots elongate as thickness is increased, putting an upper limit on the possible thickness of particle $P$.

Image processing successfully produced the phase, shape, size and orientation of the particle, and image simulation confirmed the structure and set an upper bound on the thickness of the particle. 


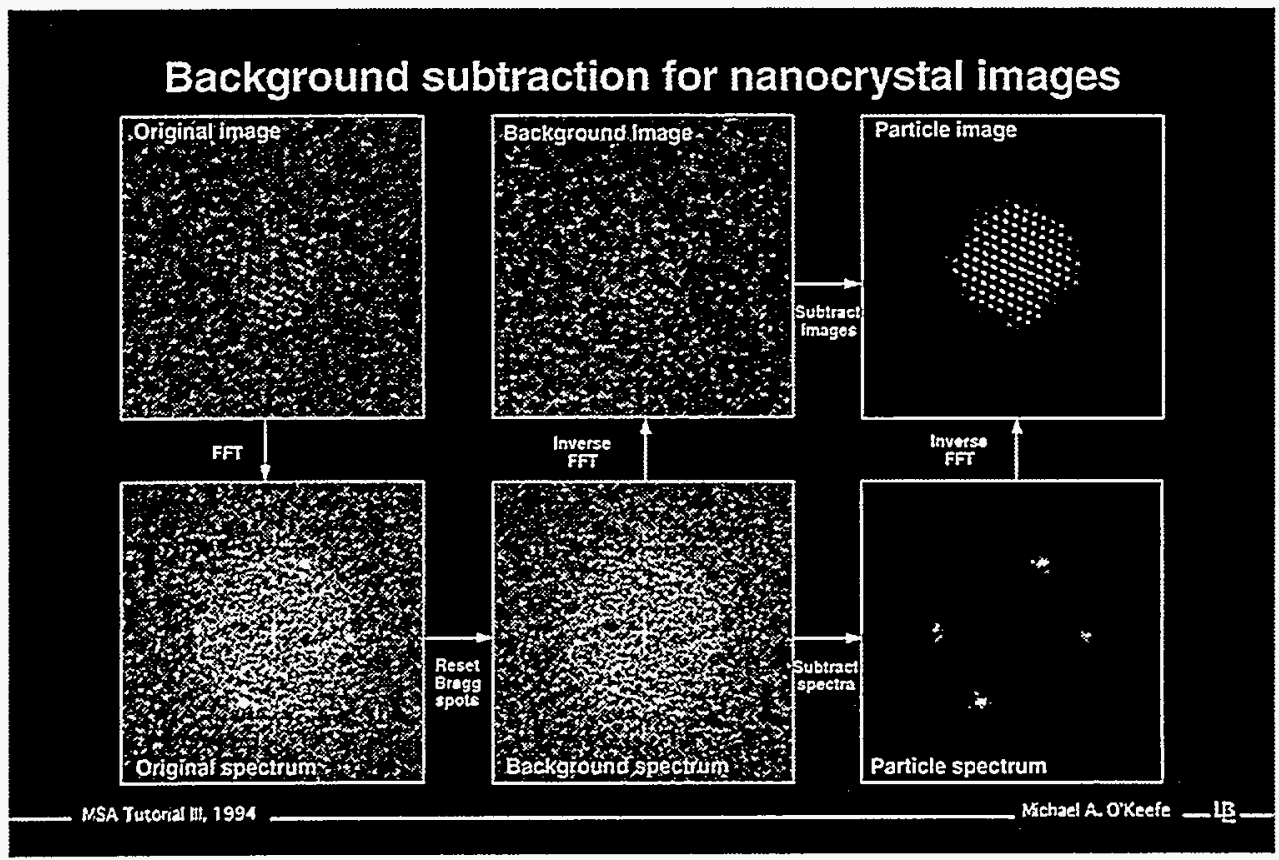

\section{Pre-processing before simulation -- the "background subtraction" technique.}

Images of a chunk of interstellar meteorite showed small crystallites embedded in a non-crystalline matrix. The problem was to characterize the crystals -- they turned out to be small particles of diamond.

The method of "background subtraction" was applied in order to remove the non-crystalline contribution to the image. An image area was FFTed, producing the diffractogram or image intensity spectrum; this spectrum contained both periodic signals (sharp spots) and a non-periodic contribution (diffuse "noise"). The sharp ("Bragg") spots were set to the local background, and an IFT performed to produce a "background" image without the periodic contribution from the crystallite. The "background" image was then subtracted from the original to produce the periodic signal. Note that the subtraction could have been performed before the IFT; in this case the intensity spectrum of the particle is made available.

The background-subtraction method has the advantage, over windowing methods of Fourier-space filtering, that it preserves the shape of the crystalline particle image by preserving the shapefunction in Fourier space.

"Enhancement of structure of interstellar diamond by image processing", M.A. O'Keefe, D. Blake, F. Freund, C.J.D. Hetherington, and J.H. Turner, in 46th Ann Proc. EMSA, Milwaukee, Wisconsin (1988) 836-838. 


\section{Structure Determination by Image Matching}

1. Compute a WPO series of images covering the resolution range of the microscope. These images represent an unattainable ideal.

2. Compute a focus series for a thin crystal (thin enough to give WPO-like character at Scherzer defocus) under conditions for this microscope.

3. If the specimen does not damage too rapidly, obtain an experimental focus series of images (as in the mullite example), including a Scherzer-defocus image. If beam damage is a problem (as for ytrium di-silicate), record the image that provides the clearest information (usually the Scherzer-defocus image), identifying it from the simulated focal series. Record all experimental conditions, including a diffraction pattern with the beam set for imaging and the objective aperture in, also a standard SAD pattern to indicate crystal tilt (as in the Takeuchite example).

4. If any recorded experimental conditions (such as beam convergence, aperture size, crystal tilt, or defocus) are different from those used in the pre-experiment computations, then recompute.

5. Match the focal series at the thin edge of the sample. Select a few images (at least one) that match for the thin area, compute a thickness series, and match images through thickness.

6. If necessary, repeat the process in a number of zone-axis orientations.

The best approaches for structure determination by image matching will vary from case to case, but the procedure given above is a good basic approach to the problem.

Experience with simulations, and with common values for the parameters of the microscope in use, will make it easier to deduce what effects changes in the various parameters will have on the character of the resultant image -- also which settings produce the image with the most information.

One problem that can arise is when values of all parameters were not recorded at the time the images were. In particular, thickness is very difficult to measure; it is often helpful to write down a value for the specimen thickness in the area under consideration -- or at least an impression -- a good guess is better than no data.

A focus series of images always contains more information than a single image, and matching of a focal series tests the model structure at more spatial frequencies. It also tests the accuracy of the microscope parameters, correcting any tendency to offset one against another.

Matching with thickness is a very good test of a model structure. Small differences in atom positions are not detectable in thin-crystal images, but are magnified by dynamical diffraction as thickness is increased. Unfortunately, so are the effects of minor crystal tilt. Aperture displacement effects and misalignment effects also become more important.

For ultimate stringency, image matching should be carried out in several orientations along major crystal axes. 


\section{- Outline -}

What use is HRTEM image simulation?

History -- image simulation was conceived for structure determination.

Uses -- the uses of image simulation and image processing - structure determination is only one of five uses for image simulation.

Structure determination - recipes and examples of structure determination by matching of experimental images and simulated ones.

How does HRTEM image simulation work?

- WPO -- interpreting images with the weak phase-object approximation; Why do some images "look like" the structure?

Beyond WPO -- the steps involved in image simulation; modelling the high-resolution TEM in the computer; computing dynamical diffraction; computing lens effects.

Using HRTEM image simulation

Running simulations -- obtaining values of simulation parameters.

Handling defects -- what additional precautions are necessary?

\section{Theory-}

- WPO. Many images "look like" the "structure". Just what is the relationship between a thincrystal-Scherzer-defocus image and the arrangement of atoms within the specimen?

- The WPO approximation lets us see how such an image is built up from image components that are linearly-related to specimen structure factors -besides, WPO images can be computed very quickly. 


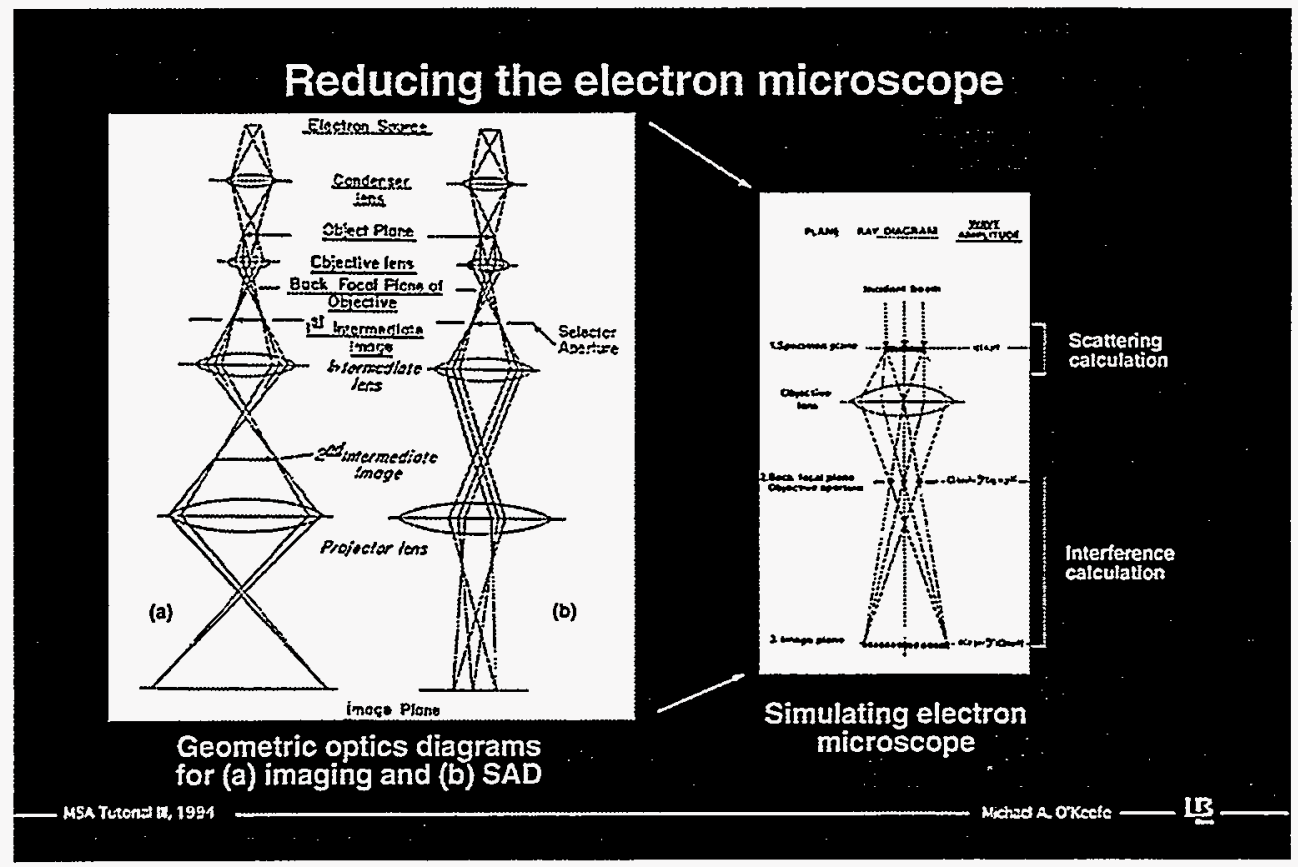

The geometrical-optics views of the TEM (left) show the main points of interest in imaging mode (a) and diffraction mode (b). An electron source is focussed onto the specimen by one or more condenser lenses. The electrons pass through the specimen, and the specimen exit-surface wave becomes the object for the imaging system. Diffracted beams leaving the specimen are brought together by the objective lens to cross at the back-focal plane of this lens, and continue on to form the first intermediate image.

In imaging mode (a), the intermediate lens is focussed so that its object plane is at the position of the first intermediate image (the image plane of the objective lens). The intermediate lens then forms the second intermediate image at its image plane. The projector lens is focussed so that its object plane is at the second intermediate image plane, and it forms the final image in its image plane.

In diffraction mode (b), the intermediate lens is focussed so that its object plane is at the position of the back focal plane of the objective lens. The intermediate lens then forms an "image" of the diffraction pattern at its image plane. The projector lens is focussed so that its object plane is at the intermediate image plane, and it forms the final image of the diffraction pattern in its image plane.

Note that a modern HRTEM will contain additional condenser lenses for better illumination control, and more intermediate lenses for better control of magnification and rotation of the image.

The view on the right is of a "reduced" TEM. It shows the essential stages in the formation of the high-resolution image, and the steps required to form a simulated image.

We have reduced the HRTEM to three items, an illuminating electron beam, a specimen, and an objective lens. We do not have to treat other lenses since they can be designed not to introduce modifications into the primary image that is formed by the objective lens. But the objective lens forms the primary image from diffracted beams leaving the exit surface of the specimen and must accept them at their Bragg angles, thus contributing spherical aberration.

Scattering We first use dynamical scattering theory (usually multislice) to compute the object transmission function and thus the exit-surface wave- function.

Interference We allow the diffracted beams to interfere via an auto-correlation of the image amplitude components to produce the image intensity spectrum, and hence the image intensity as it appears in the image plane of the lens.

Thus the simulation calculation has two major parts: (1) computing the dynamical scattering from the model object, and (2) computing the image intensity from the exit-surface wave. 


\section{What information do diffracted beams carry?}

1. Incident electrons scatter from the crystal potential ọ $(x, y, z)$.

2. In reciprocal space, the three-dimensional potential $\phi(x, y, z)$ can be described in terms of its Fourier components V(hkl).

$$
\phi(x, y, z)=\sum_{h} \sum_{k} \sum_{1} V(h k 1) \cdot \exp \{2 \pi i(h x+k y+l z)\}
$$

3. For high energy electrons the Eusald sphere is (almost) planar, and diffracted beams come from the V(hkO) section of the reciprocal-space lattice.

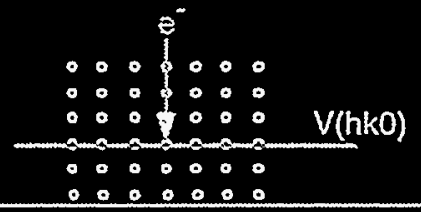

4. A section in reciprocal space is a projection in real space.

$$
\phi_{p}(x, y)=\sum_{h} \sum_{k} V(h k 0) \cdot \operatorname{cxp}\{2 \pi i(h x+k y)\}=\int_{0}^{H} \phi(x, y, z) \cdot d z
$$

5. At the crystal exit surface, the electron wave carries information about the crystal potential projected in the direction of the incident electron beam.

We want to know how the image is related to the structure. It turns out that what the incident electrons "see" is the crystal potential created by the presence of the atoms and their bonding electrons. This potential will be periodic for crystalline specimens, and aperiodic for defects and amorphous substances; in any case, the electrons scatter from the potential. But the potential that they "see" is described (mostly) by only a section of the reciprocal-space lattice. This is equivalent to the electrons experiencing scattering from a projection of the three-dimensional potential within the specimen; this projection is in the direction of the incident beam. For a specimen of thickness $\mathrm{H}$ in the beam direction, the scattering potential is a projection (integral) from zero to $\mathrm{H}$ of the complete three-dimensional potential.

And the exit-surface wave carries information about the projected potential. 


\section{How is the image related to the structure? -- by WPO.}

Image formation in the electron microscope occurs in two major steps

- Scattering -- electron waves propagate through the specimen, scattering from the specimen potential and thus carrying structural information.

- Interference -- electron waves from the exit-surface of the specimen are brought together by the lens system and interfere to produce an image.

To interpret the image, we need to understand its relationship to the structure. But, in general, the relationship is not linear. We have

- Dynamical Scattering -- in the scattering step, electrons typically scatter many times within the specimen.

- Multiple Interferences -.- the image intensity spectrum is formed by the interference of each diffracted beam with every other diffracted beam.

The Weak-Phase Object Approximation -- the image/structure relationship is (approximately) linear when the specimen is thin. We require --

- Kinematical Scattering - when the specimen is thin, the proportion of singly-scattered electrons is much greater than those multiply scattered.

- Linear interferences - when the central beam is strongest, then the image intensity spectrum is formed linearly by two interferences per component.

In order to get an understanding of the relationship between the image and the specimen structure, we can use the WPO or weak phase-object approximation [1].

Scattering -- Under the WPO approximation, the specimen scatters so weakly (or is so thin) that the proportion of multiple scattering is very small compared with the number of singly-scattered electrons emerging from the specimen. We can approximate the full dynamical scattering theory by replacing it with a kinematical theory. Then the diffracted beam amplitudes are linearly related to the specimen structure factors.

Imaging -- Under the WPO approximation, the diffracted beam intensities are much less than that of the central beam. Thus all diffracted beam interferences may be neglected except for the strong interferences between the central beam and each of the other beams. Then the image intensity spectrum is linearly related to the image amplitude spectrum and to the diffracted beam amplitudes [2,3].

[1] J.M. Cowley \& S. lijima, "Electron microscope image contrast for thin crystals", Z. Naturforsch. 27a (1972) 445-451.

[2] "Electron image simulation; a complementary processing technique", M.A. O'Keefe in Proc. 3rd Pfefferkorn Conf. on Electron Optical Systems, Ocean City, Maryland (1984) 209-220.

[3] "Resolution in high-resolution electron microscopy", M.A. O'Keefe, Ultramicroscopy 47 (1992) 282-297. 


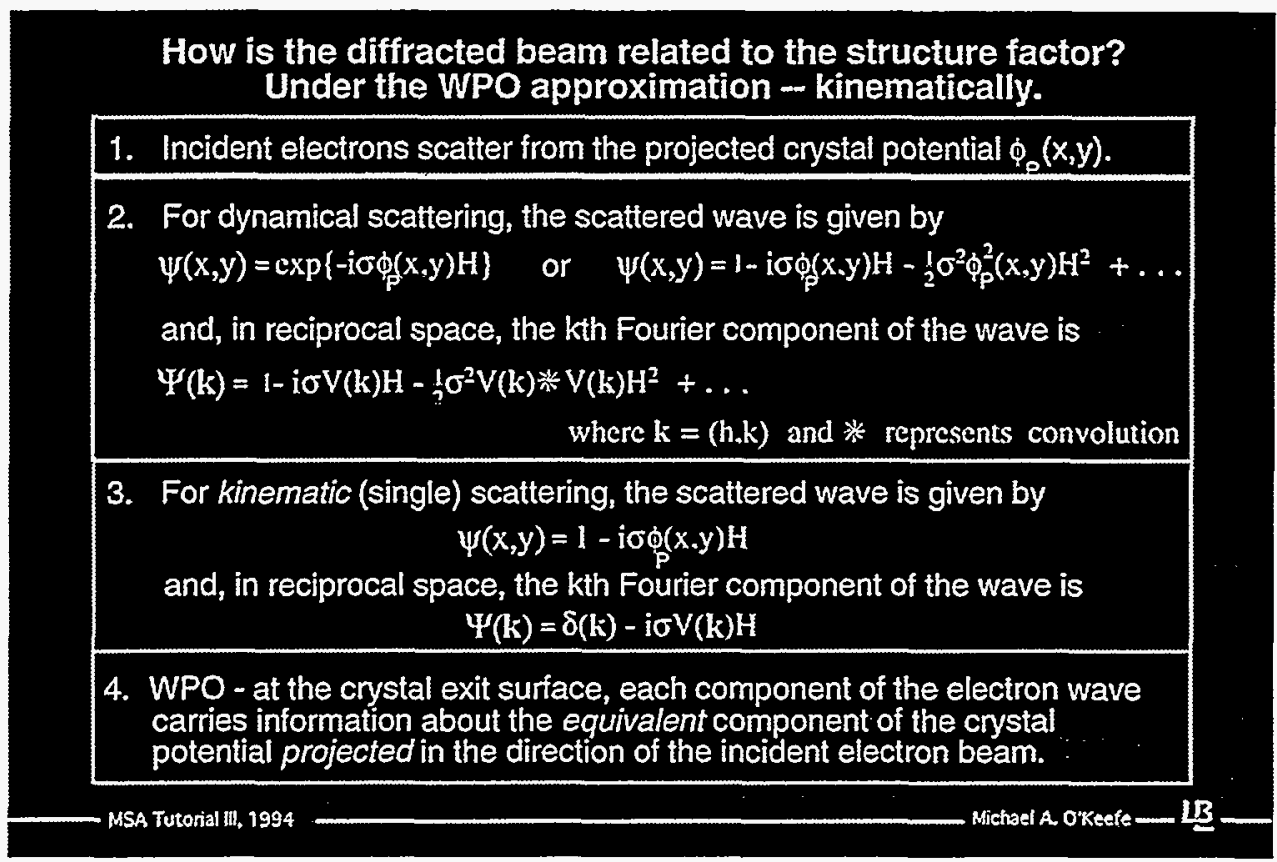

\section{Towards the WPO approximation -}

Dynamical Scattering - We know that the image carries information about the projected potential, but how is that information distributed? It turns out that for dynamical scattering, each diffracted beam $\Psi(\mathbf{k})$ carries information about every component $V(\mathbf{k})$ of the projected potential. The exponential in real space (equal to an infinite series of multiplications of $\phi(x, y)$ ) is equivalent to an infinite series of convolutions of $V(\mathbf{k})$ in reciprocal space. Although the term in $V(\mathbf{k})$ represents single scattering, that in $V(\mathbf{k}) * V(\mathbf{k})$ indicates double scattering, with every $V(\mathbf{k})$ mixing with every other $V(\mathbf{k})$ for all values of $|\mathbf{k}|$. And additional terms produce triple scattering, $\mathrm{V}(\mathbf{k}) * \mathrm{~V}(\mathbf{k}) * \mathrm{~V}(\mathbf{k})$, and higher.

Kinematical Scattering - In the case of thin specimens, we can make a kinematical approximation that each electron scatters only once. This means that we can truncate the expansion of the exponential of $\{-i \sigma \phi(x, y) H\}$ after two terms as $1-i \sigma \phi(x, y) H$. Then the electrons in the kth diffracted beam have scattered from only the kth component of the specimen potential. And each component of the exit surface wave (i.e. each diffracted beam) carries information about its corresponding (projected) potential component, with no mixing of information from other components.

Note: We make a kinematical approximation here as the first step in deriving an expression that will allow us to investigate the weak-phase-object (WPO) type of image. Regular (full) image simulations always require a full dynamical computation using multislice or Bloch-waves. 
Formation of the image intensity spectrum

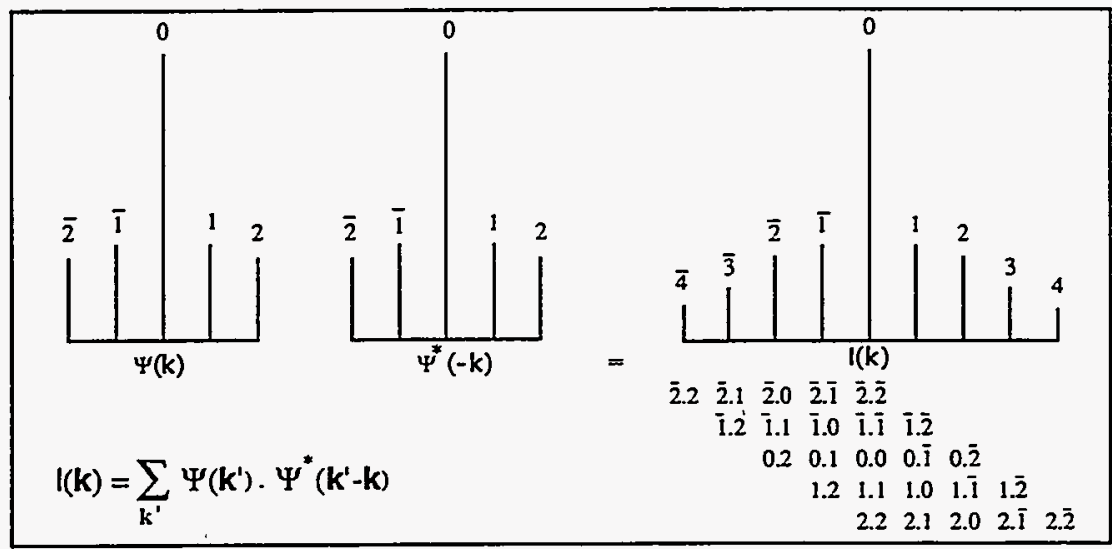

In this illustration, five diffracted beams $\Psi(\mathbf{k})$, interfere to produce nine intensity-spectrum terms l(k). Interference pairs are shown.

— MSA Tutorial III, 1994 __ Michael A. O'Keefe —

Image intensity spectrum (general case) --

How is the image intensity spectrum related to the image amplitude spectrum (i.e. to the diffracted beams after their lens-imposed phase changes)?

The image intensity spectrum (the Fourier transform of the image intensity) is formed from the image amplitude spectrum (the diffracted beams after phasing by the objective lens) by a series of interferences that can be described with an auto-correlation operation.

The diagram illustrates the auto-correlation for the case of five co-linear beams allowed through the objective aperture.

In the general case, every diffracted beam interferes with every other, and the intensity spectrum components consist of the sums of from one to five interference-pairs (it would be more than five if more than five beams had been admitted by the objective aperture). The auto-correlation can be written as the convolution --

$$
\mathrm{I}(\mathbf{k})=\sum_{\mathbf{k}^{\prime}} \Psi\left(\mathbf{k}^{\mathbf{1}}\right) \cdot \Psi^{*}\left(\mathbf{k}^{\prime}-\mathbf{k}\right)
$$

"Resolution-damping functions in non-linear images", M.A. O'Keefe, in 37th Ann. Proc. EMSA, San Antonio, Texas (1979) 556-557.

"Electron image simulation; a complementary processing technique", M.A. O'Keefe in Proc. 3rd Pfefferkorn Conf. on Electron Optical Systems, Ocean City, Maryland (1984) 209-220. 


\section{Formation of the linear-image intensity spectrum}

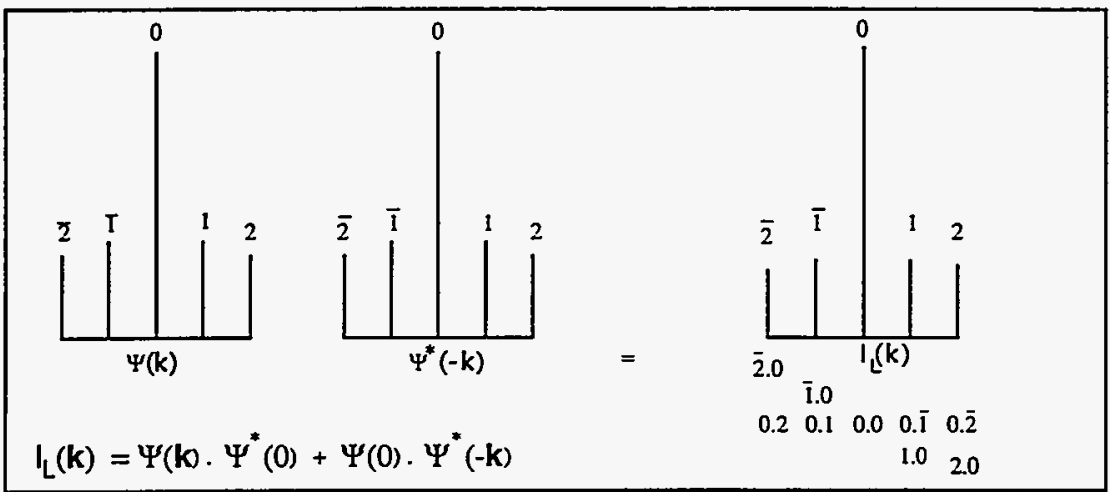

Under linear-image conditions, five diffracted beams $\Psi(\mathbf{k})$ interfere to produce only five intensity-spectrum terms $I_{L}(\mathbf{k})$. Each intensityspectrum term now consists of the sum of only two interference pairs -- $\Psi(\mathbf{k}) \cdot \Psi^{*}(0)$ and $\Psi(0) \cdot \Psi^{*}(-\mathbf{k})$.

Image intensity spectrum (linear-image case)-

In the case of a thin crystal, only interferences with the central beam are considered in the formation of the image intensity spectrum (because other beams are much weaker than the central beam, and interference-pairs that do not include the central beam will be much weaker than those that do). In this case each intensity spectrum component (except for the zero component) consists of the sum of just one pair of interference-pairs. The kth intensity spectrum component $I(\mathbf{k})$, will consist of an interference between the kth diffracted beam and the central beam plus another between the central beam and the complex conjugate of the minus-kth beam --

$$
\mathrm{I}_{\mathrm{L}}(\mathbf{k})=\Psi(\mathbf{k}) \cdot \Psi^{*}(0)+\Psi(0) \cdot \Psi^{*}(-\mathbf{k})
$$

"Resolution-damping functions in non-linear images", M.A. O'Keefe, in 37th Ann. Proc. EMSA, San Antonio, Texas (1979) 556-557.

"Electron image simulation; a complementary processing technique", M.A. O'Keefe in Proc. 3rd Pfefferkorn Conf. on Electron Optical Systems, Ocean City, Maryland (1984) 209-220. 


\section{The weak-phase object (WPO) approximation}

Under weak-phase object concitions (weak scattering) the exit-surface wave -

$$
\psi_{E}(x)=1-i \sigma \Phi_{p}(x) H
$$

produces diffracted beams $\Psi_{E}(k)$ that depend only on the corresponding $V(k)$ -

$$
\Psi_{E}(k)=\delta(k)-i \sigma V(k) H
$$

Also, each image intensity spectrum component has only two interierences -

$$
I(k)=\Psi(k) \cdot \Psi^{*}(0)+\Psi(0) \cdot \Psi^{*}(-k)
$$

so the kth image intensity spectrum component is given by-

$I(k)=\delta(k)+2 \sigma V(k) H \sin \gamma(k)$

At "Scherzer" defocus, where $\sin \chi(k)=-1$ for all $k$

$$
l(k)=\delta(k)-2 \sigma V(k) H
$$

and the WPO image intensity is -

$$
l(x)=1-2 \sigma \phi(x) H
$$

\section{Algebraic derivation of the WPO image expression}

Under WPO conditions, the kth component in the "linear-image" intensity spectrum consists of only the two interference pair $s$ in equation (3) above -

$$
l(k)=\Psi(k) \cdot \Psi^{\star}(0)+\Psi(0) \cdot \Psi^{\star}(-k)
$$

and, since $\Psi(0)$ has a weight that is very close to unity for a thin crystal,

$$
I(k)=\Psi_{E}(k) \cdot \exp [i \chi(k)]+\Psi_{E}^{*}(-k) \cdot \exp [-i \quad \chi(-k)]
$$

where the image amplitude term $\Psi(k)$ is just the exit-surface term $\Psi_{E}(k)$ multiplied by the lens phase factor $\exp [i \chi(k)]$.

Under the kinematic scattering approximation, the exit-surface wavefunction in direct space may be written

$$
\psi(x)=1-i \sigma \phi(x) H
$$

where $\sigma$ is the interaction coefficient, $\phi(x)$ is the specimen potential projected in the direction of the incident electron beam, and $H$ is the specimen thickness through which the projection is taken.

Transforming to Fourier space -- $\quad \Psi_{E}(k)=\delta(k)-i \sigma V(k) H$

Substituting (2) into (3a)

$$
I(k)=\delta(k)-i \sigma V(k) H \exp [i \chi(k)]+i \sigma V^{*}(-k) H \exp [-i \quad \chi(-k)]
$$

Now $V^{*}(-k)=V(k)$ for kinematic scattering, and $\{\exp [-i \chi(-k)]-\exp [i \chi(k)]\}$ is equal to 2 isin $\chi(k)$, so the image intensity spectrum component is then

$$
l(k)=\delta(k)+2 \sigma V(k) H \cdot \sin \chi(k)
$$

and the magnitude of the kth term in the image intensity spectrum is just proportional to $V(k)$, the kth Fourier coefficient of the projected potential, and to $\sin \chi(k)$ at the corresponding value of $k$. At Scherzer focus, $\sin \chi(k)=-1$ giving (5); Fourier transformation yields the image intensity (6). 
The significance of $\sin \chi$ in forming WPO images

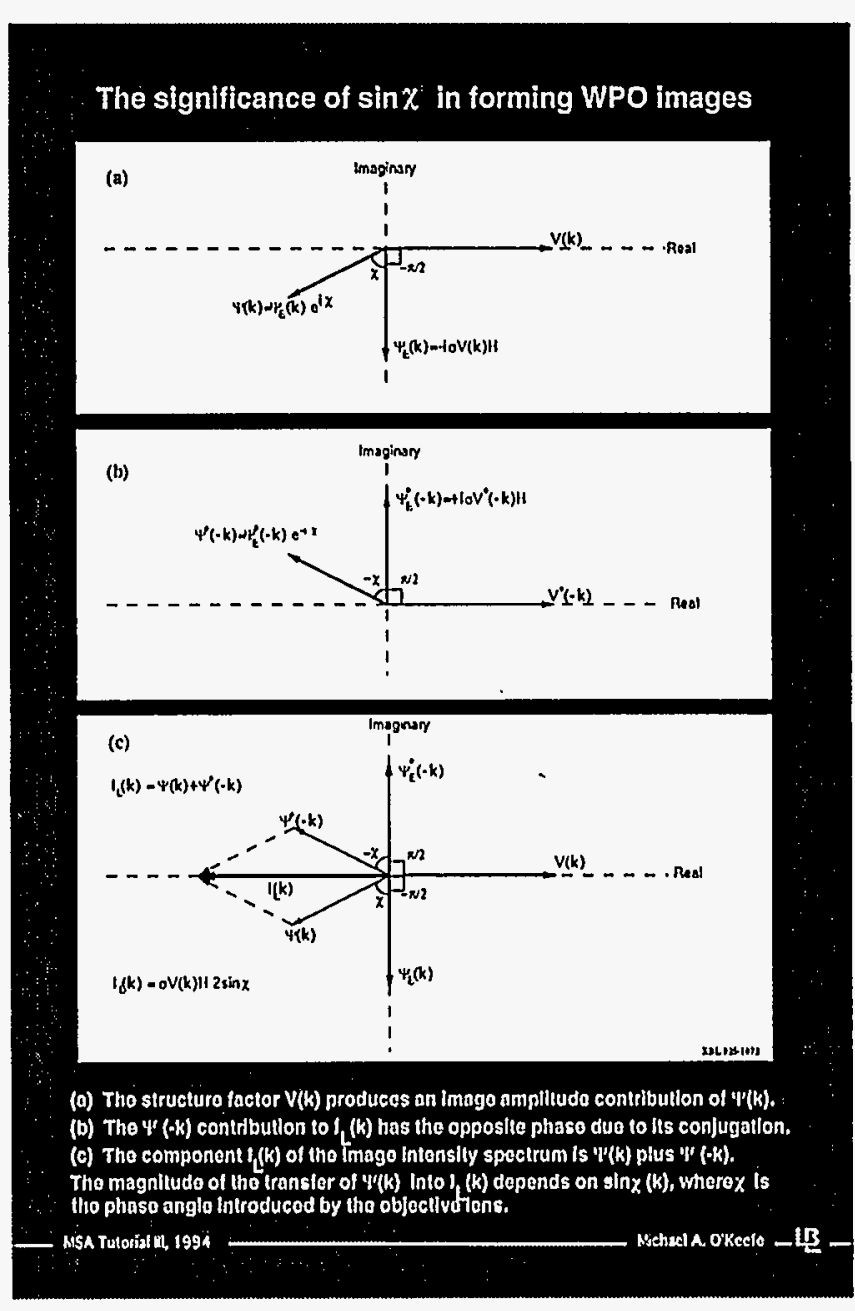

'Resolution in high-resolution electron microscopy", M.A. O'Keele, Uliramicroscopy 47 (1992) 282-297.
The action of $\sin \chi$ can be described in the complex (Argand) plane. The figure shows the formation of the kth linear-image intensity spectrum component $I_{L}(k)$ from the $k$ th structure factor $V(k)$.

(a) The structure factor $V(k)$ (assumed to be real, i.e for a centrosymmetric struclure) can be represented by a vector lying along the real axis in the complex plane (this construction works for non-centro structures, but is more difficult to draw since the $V(k)$ is no longer along the positive real axis). Single (kinematic) scattering produces an exit-surface wave component (or diffracted beam) that is rotated clockwise by $\pi / 2$ from $V(k)$, and that can be represented by a new vector $-f \sigma \mathrm{V}(\mathrm{k}) \mathrm{H}$ pointing in the negative imaginary direction. The exit-surface wave component undergoes a phase shift of $\mathcal{X}$ in the objective lens; thus the image amplitude component, shown as $\Psi(\mathbf{k})$, is a vector of the same length as the exit-surface vector (the same amplitude), but rotated clockwise a further $\chi$.

(b) The complex conjugate of the minus-kth image amplitude component is formed similarly.

(c) The kth image-intensity-spectrum component is just the sum of the kth amplitude spectrum component (a) and the complex conjugate of the minus-kth image amplitude spectrum component (b). It has a magnitude of $2 \sin \chi$ times the amplitude spectrum component and lies along the negative extension of the structure factor $V(k)$. Note that, because the magnitude of the intensity-spectrum component is proportional to the $\sin$ of $\chi$, it is maximized when $\chi$ is equal to $-\pi / 2$.

Because the image intensity spectrum component lies along the negative extension of its structure factor, and since the structure factor is phased to produce a high value of crystal potential near atom positions, values of $\chi$ that lie near minus $\pi / 2$ will produce "black atom" images. 
The CTF is a plot of sin $\chi$ against spatial frequency and describes transfer of information into the image

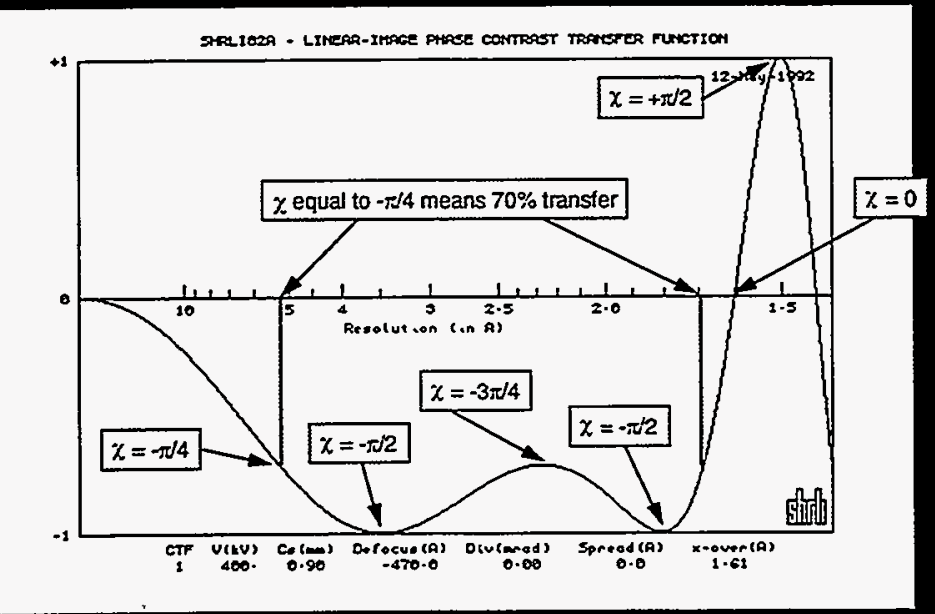

The contrast transfer function (CTF) -

- a CTF is a plot of $\sin \chi$ for a range of spatial frequencies.

- the CTF changes shape with changes in defocus.

- for any particular defocus., a CTF depicts the degree of transfer of the diffracted beams into the image intensity spectrum (the linear-image intensity spectrum) by the beams interference with the zero beam.

- the CTF does not give any information about the transfer of the non-linear interference terms occurring between non-zero beams.

- the CTF assumes that the diffracted beams are singly scattered. 


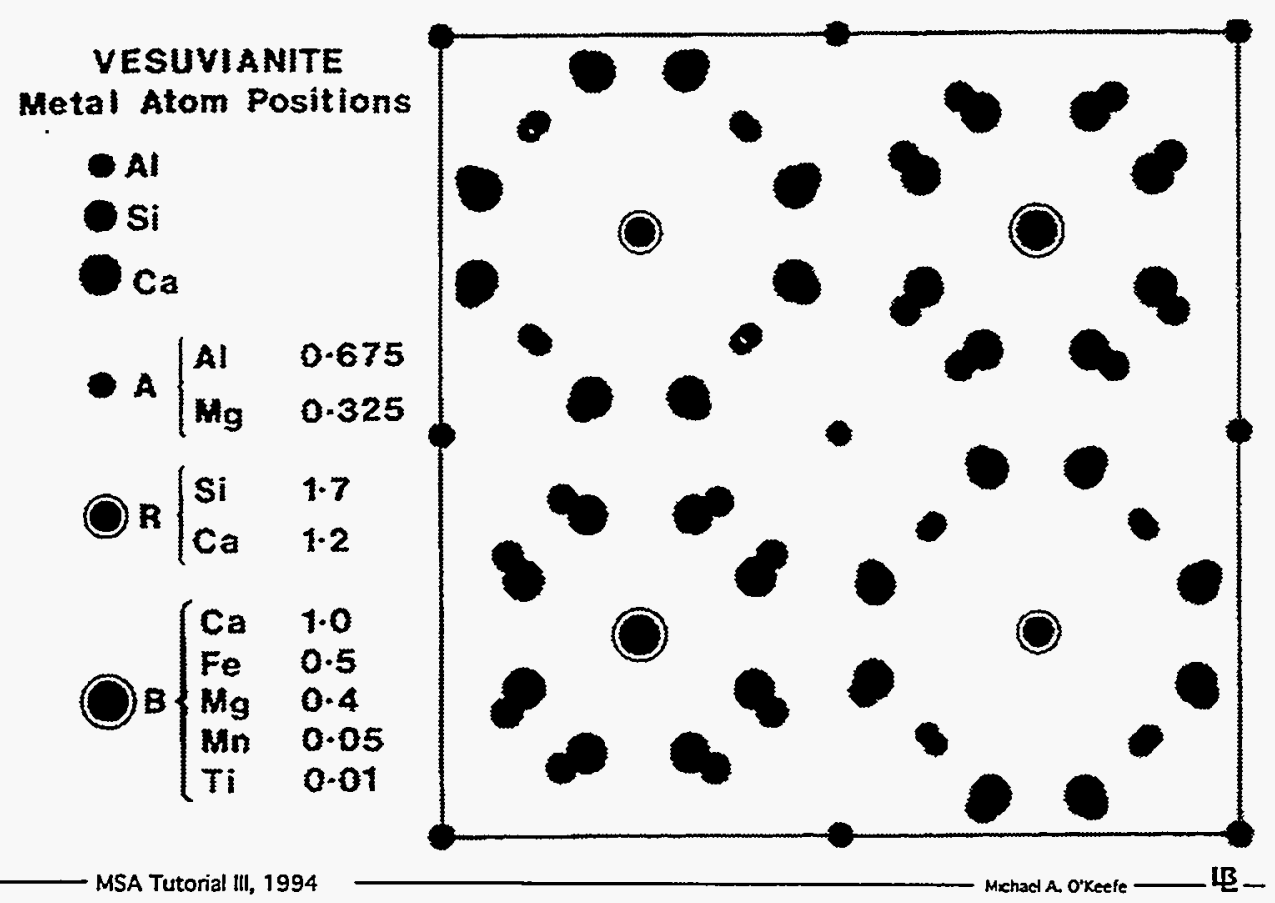

A set of images of vesuvianite provides an excellent illustration of the use of the WPO (weak phase-object) approximation. The structure of vesuvianite is shown above, viewed in [001] projection with metal atoms represented as spots with sizes approximately proportional to electron scattering power. The average scattering density is low, like most alumino-silicate minerals.

"Electron microscope image simulation", M.A. O'Keefe and P.R. Buseck, to be published. 


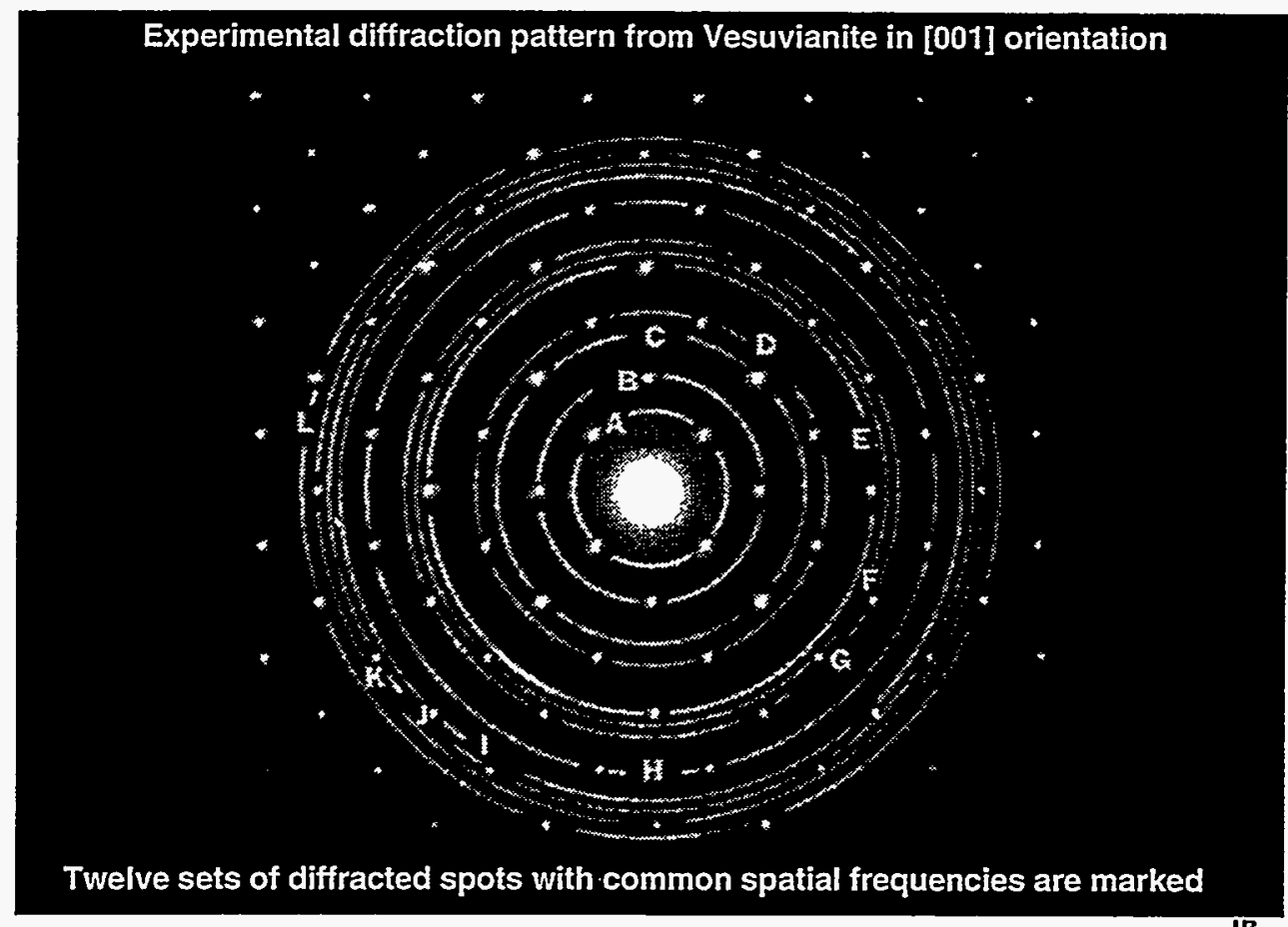

MSA Tutorial III, 1994

Machael A. O'Keele

The diffraction pattern from vesuvianite in [001] orientation shows sets of diffracted beams; each set consists of four or eight beams with identical spatial frequency. Each set of diffracted beams will contribute one image component to the image; the image component is the Fourier transform of the beam-set. The figure shows the first 12 sets of diffracted spots from vesuvianite in [001] orientation. The sets of spots are marked, from the lowest frequency $(A)$, through the twelfth $(L)$.

- "Electron microscope image simulation", M.A. O'Keefe and P.R. Buseck, to be published. 
The first twelve image components of Vesuvianite in [001] orientation

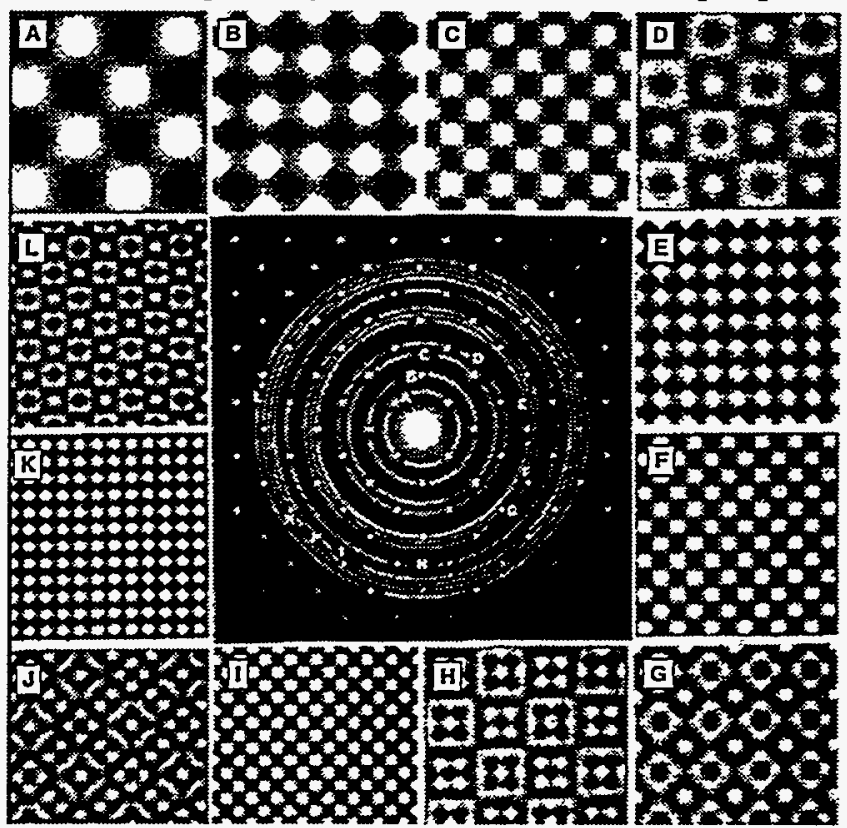

Each set of diffracted beams with identical spatial frequency will contribute one image component to the image. The figure shows the first 12 beam-sets and their corresponding image components from vesuvianite in [001] orientation.

The central (experimental) diffraction pattern has the first 12 sets of spots marked ( $A$ through $L$ ).

The computer-generated components show the patterns (image components) contributed to the HREM image by each set of beams. The A pattern is generated by the four 110 beams and appears as a checker-board pattern with large spacing. The $B$ pattern from the 200 beams has $1 / \sqrt{2}$ the spacing of the A checkerboard and is rotated $45^{\circ}$ from it. The $\mathrm{C}$ component is formed by the 220 beams and is oriented with the $A$ pattern but with half its spacing. The $\mathrm{D}$ component is more complicated because it comes from eight 310 beams (it can be viewed as the sum of two checkerboard patterns of the same spacing but rotationally offset by $37^{\circ}$ ).

Linear images are "built-up" by adding image components weighted by structure-factor amplitudes $V(\mathbf{k})$ and $\sin \chi(\mathbf{k})$, including any phase reversals due to the CTF.

WPO images are "built-up" by adding image components weighted by structure-factor amplitudes $\mathrm{V}(\mathbf{k})$ with no reversals.

Images from thicker crystals consist of the same image components, but weighted by complex dynamical scattering and lens-aberration factors.

Similar image components will occur for similar structures.

"Electron microscope image simulation", M.A. O'Keefe and P.R. Buseck, to be published. 


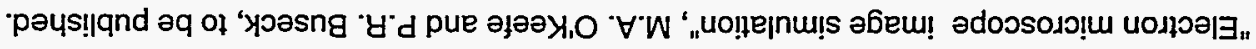

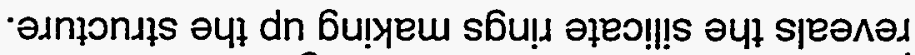

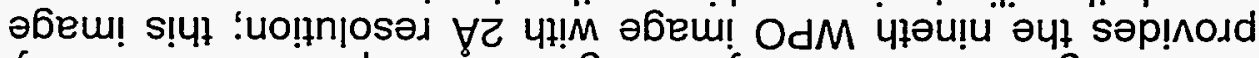

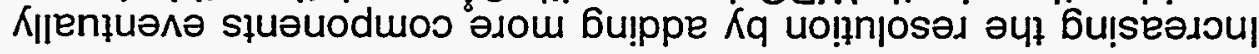

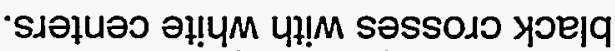

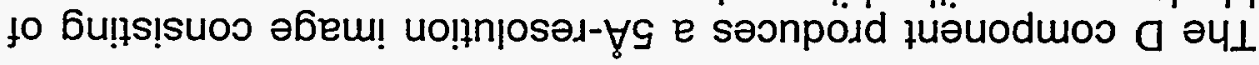

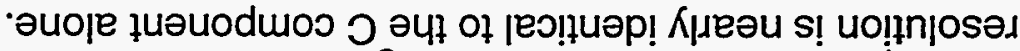
$\forall 9^{\circ} 9$ le әбeس! OdM ә4l pue '

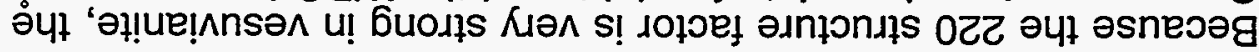

ๆ|ә0 әч१ to ұuespenb

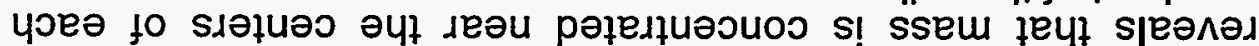

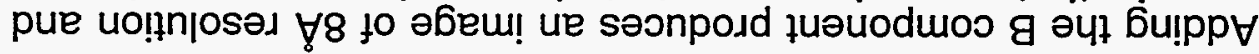

-

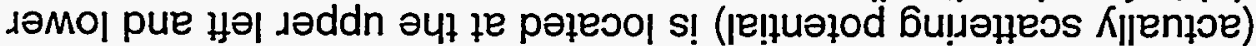

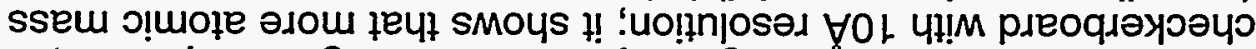

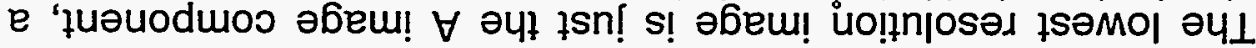

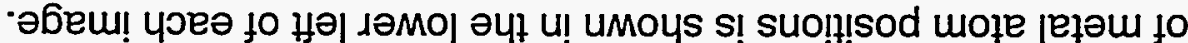

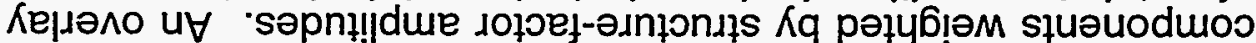

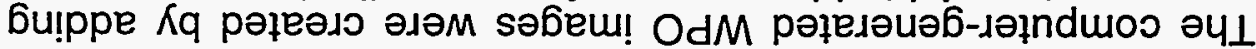

- uo!fełuə!ı

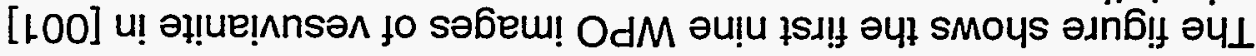

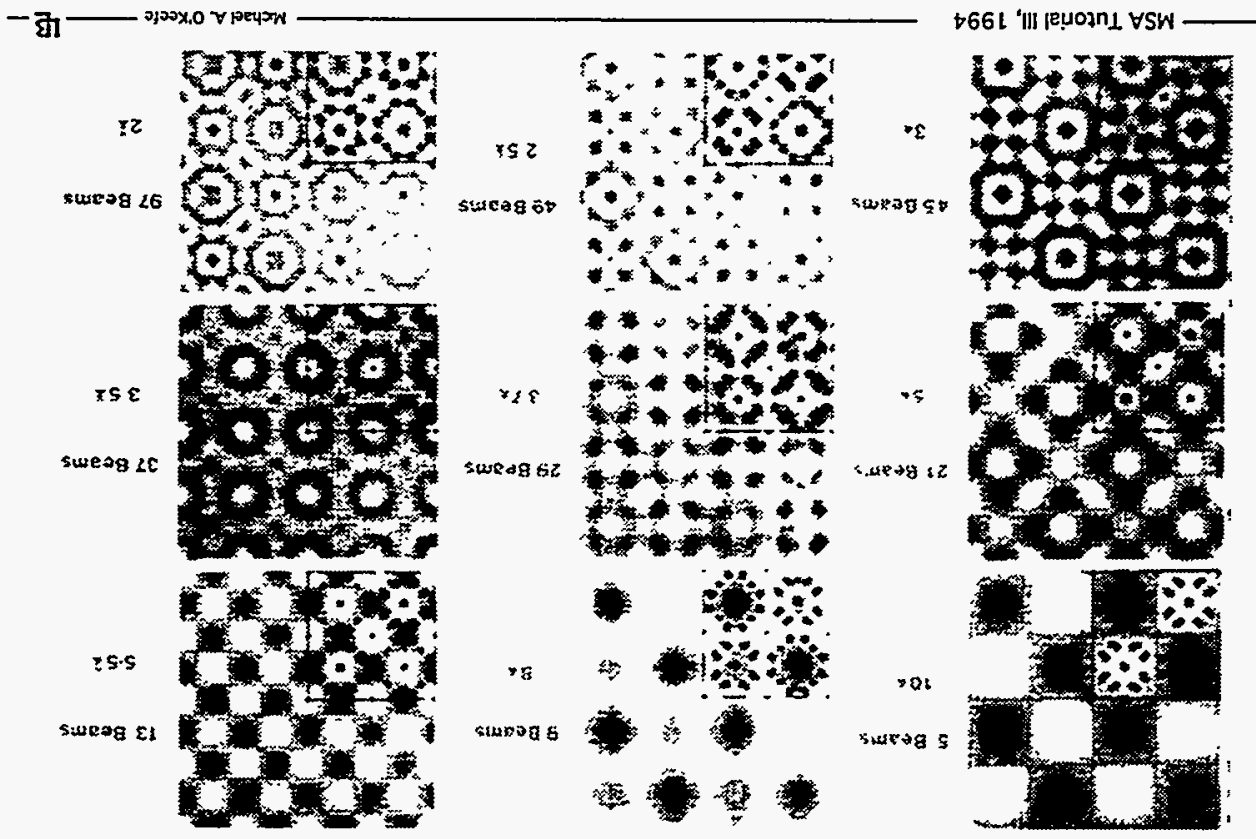

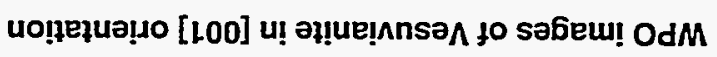




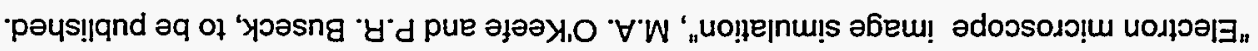

$\forall L^{\circ} \varepsilon$ to uolınjosəd e pəג!nbəג әлеч pınom

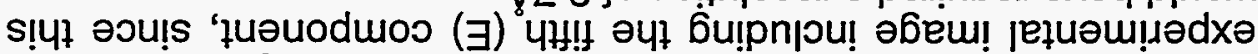

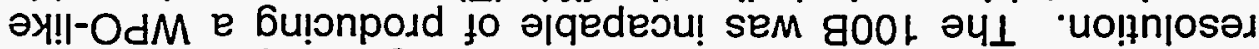

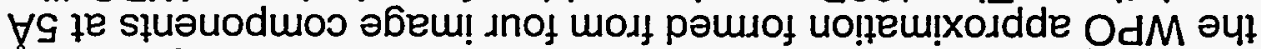

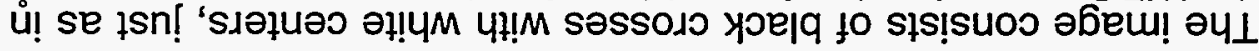

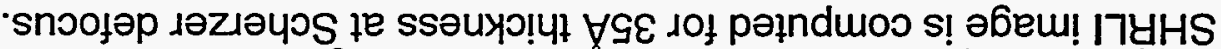

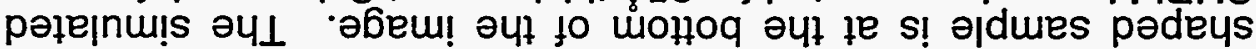

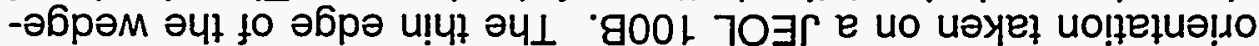

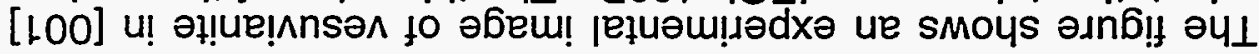

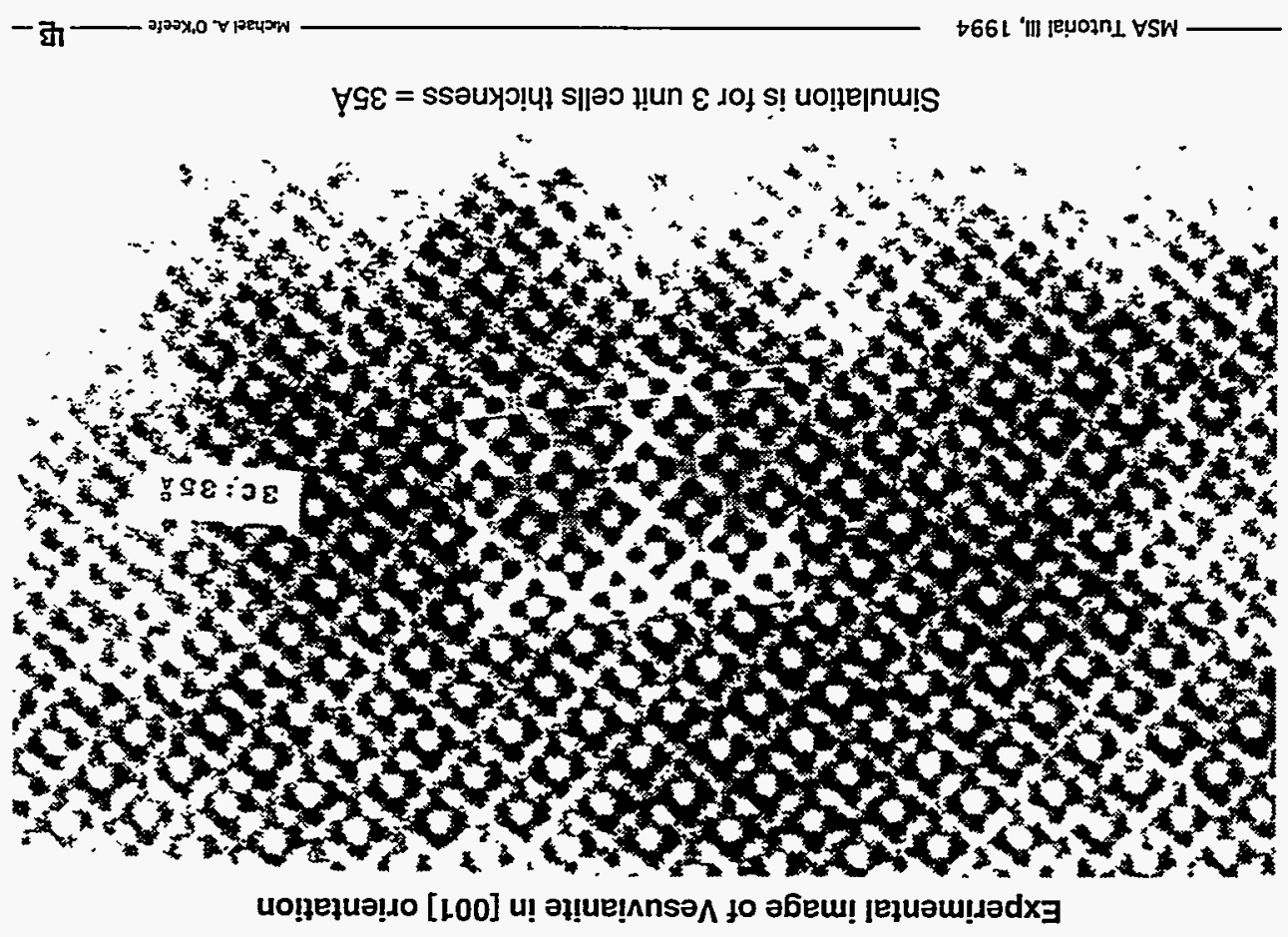




\section{Experimental image of Vesuvianite in [001] orientation}

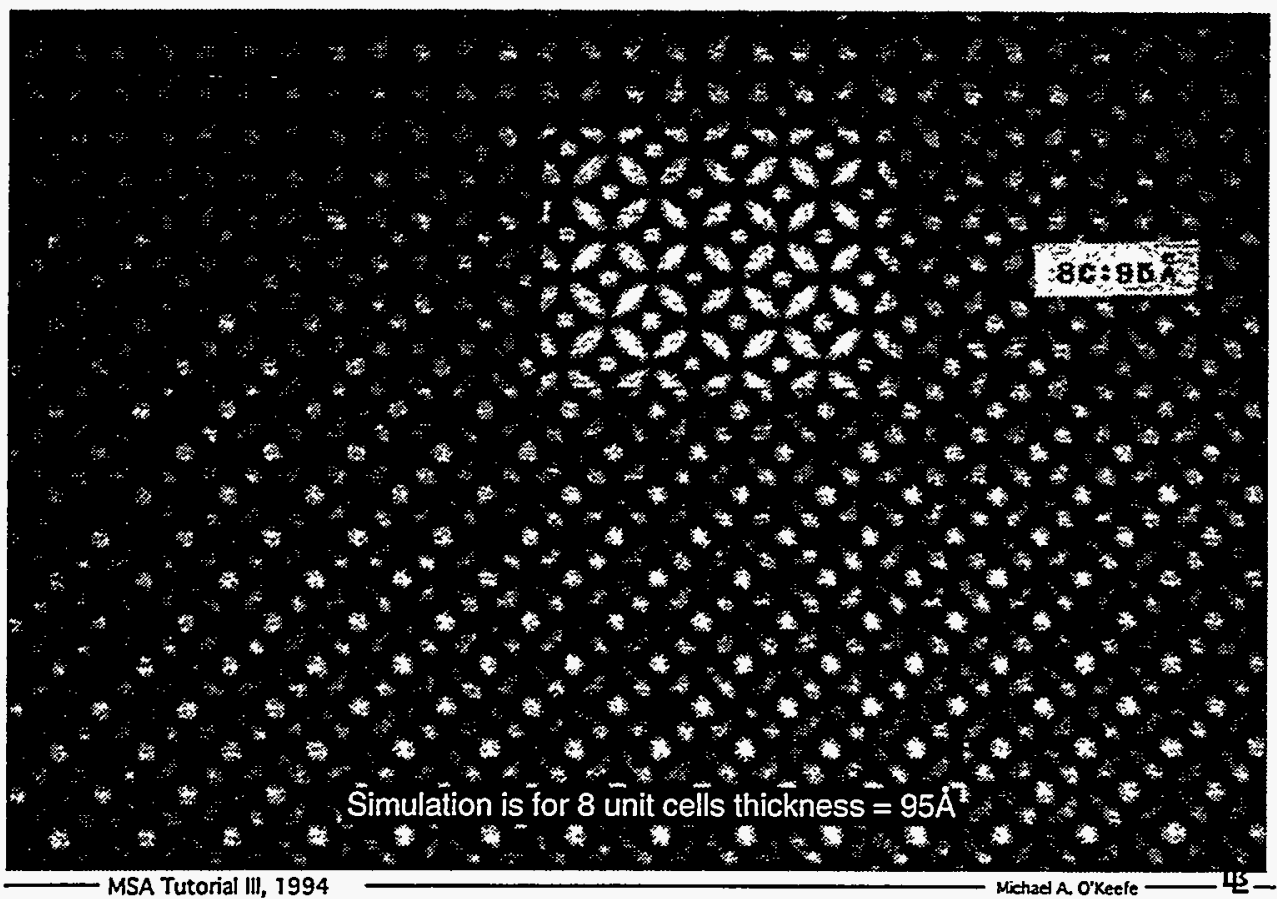

The figure shows an experimental image of vesuvianite in [001] orientation taken on a JEOL 100B. The thiner area of the wedgeshaped sample is at the bottom of the image. The simulated SHRLI image is computed for $95 \AA$ thickness.

Although the image still consists of black crosses with white centers in the lower part of the image, the thicker (upper) area appears more like a wrought-iron lattice with circular (white) holes filled with black crosses with center holes.

Such an image did not appear in the WPO approximations, and is due to non-WPO weightings of the four contributing image components

"Electron microscope image simulation", M.A. O'Keefe and P.R. Buseck, to be published. 


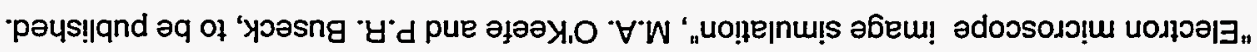

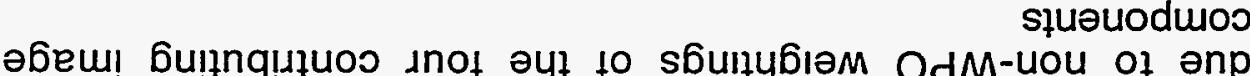
s! pue 'suo!̣eu!xoddde OdM әчl u! deədde fou p!̣ әбeu! ue yons

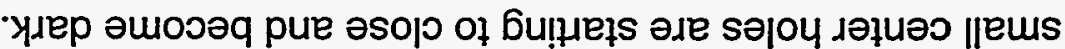

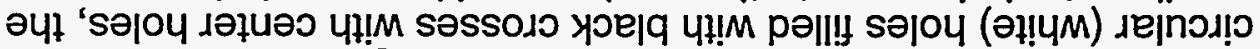

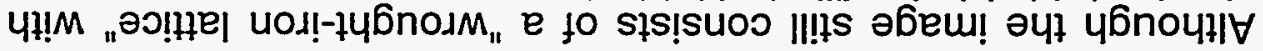

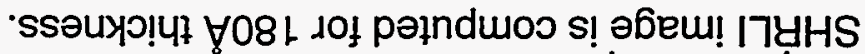

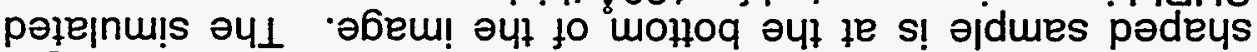

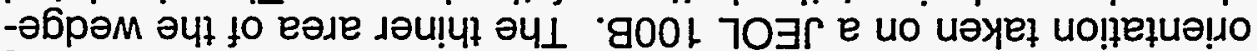

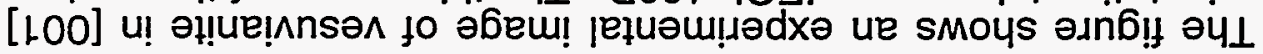

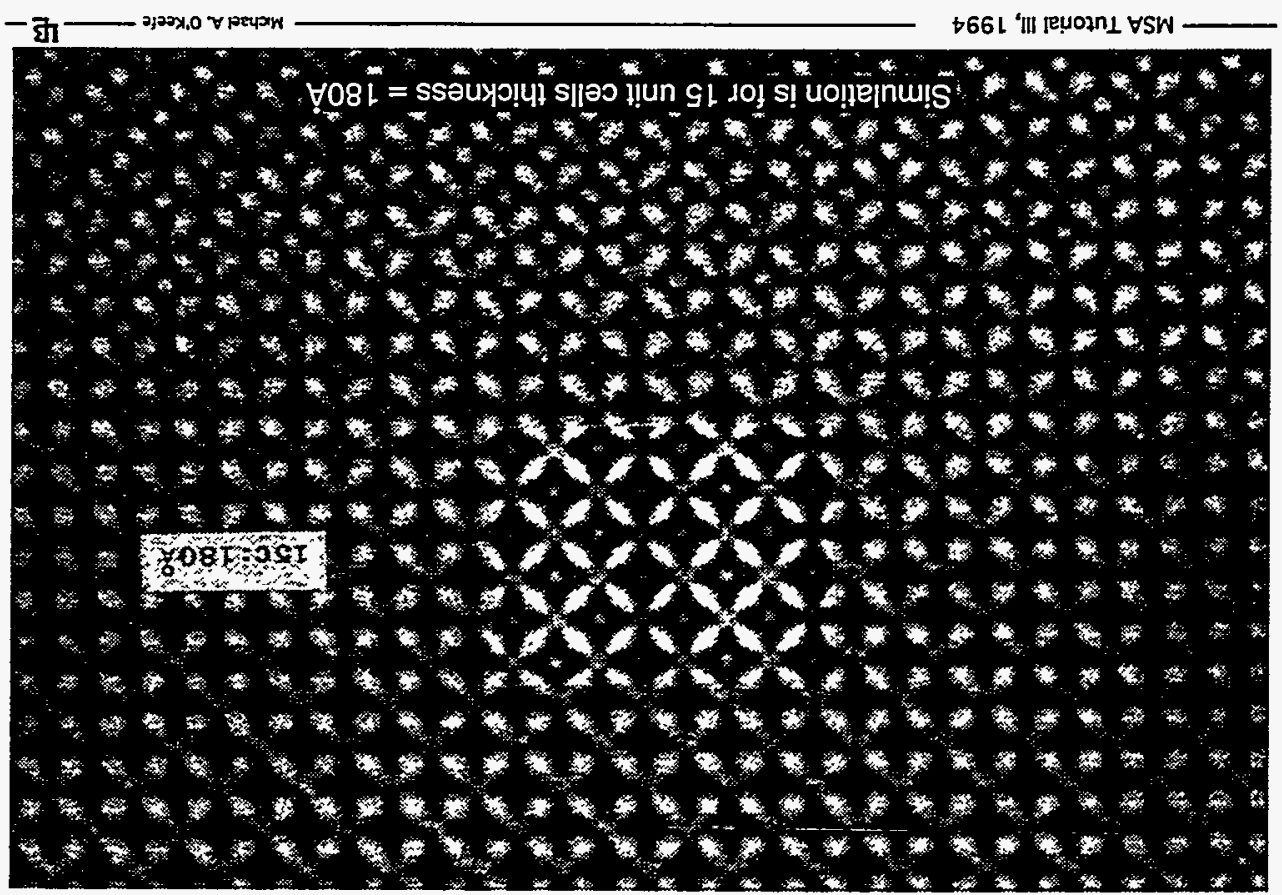

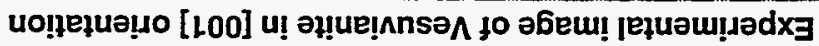




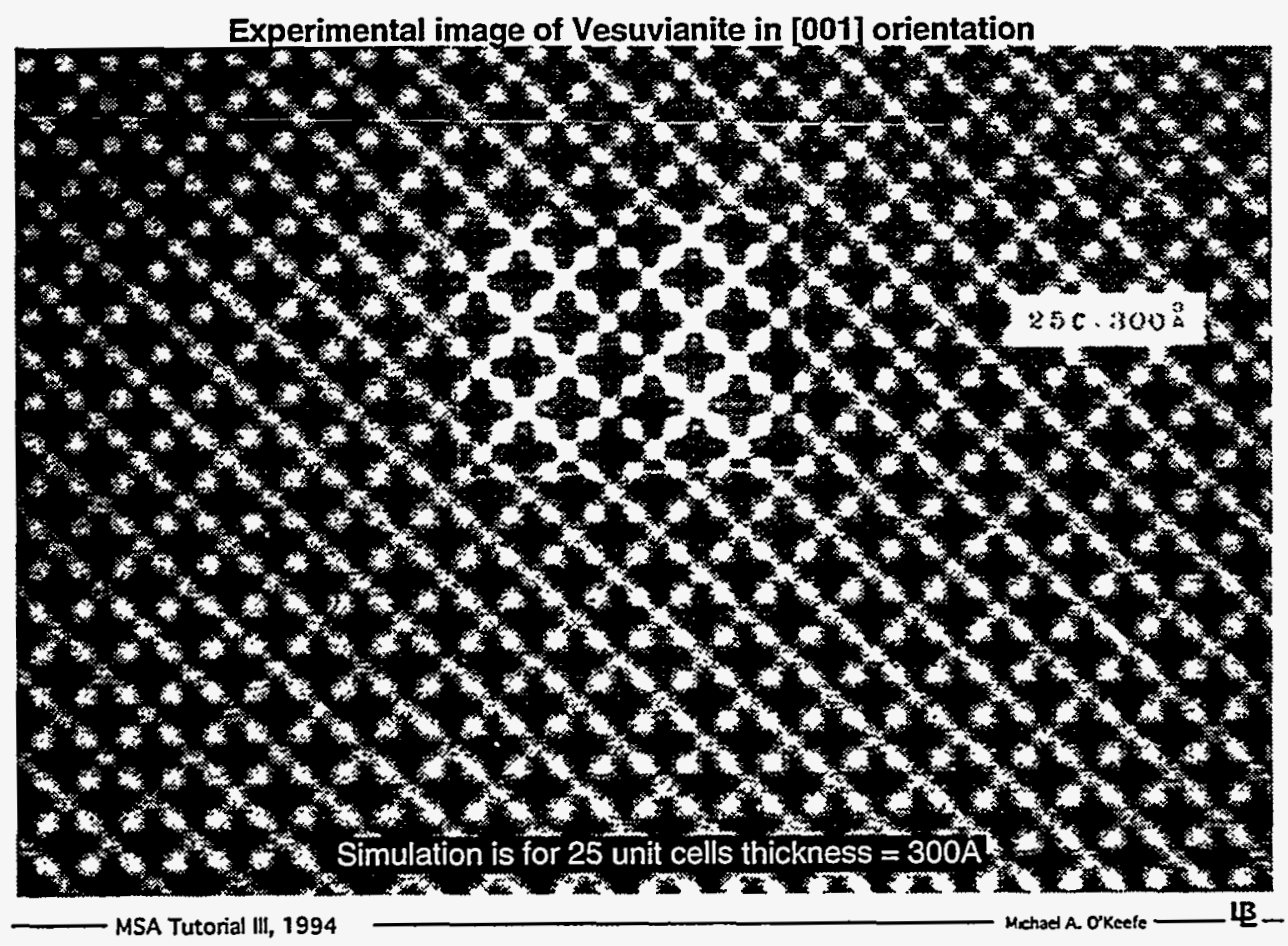

The figure shows an experimental image of vesuvianite in [001] orientation taken on a JEOL 100B. The thiner area of the wedgeshaped sample is at the bottom of the image. The simulated SHRLl image is computed for $300 \AA$ thickness.

Now the image consists of solid-black crosses with little stubby arms and no center holes.

Such an image did not appear in the WPO approximations, and is due to non-WPO weightings of the four contributing image components $A$ through $D$. In addition, some higher-frequency contributions are visible -- the small white spots in the arms of the crosses show the presence of an I image-component (consisting of 440 frequencies) that is contributing because the 220 spots (that continue to produce the $\mathrm{C}$ component by linear-image interferences with the central beam) are strong enough at this crystal thickness to produce a significant 440-type component by "nonlinear" (or "second-order") "cross-aperture" interferences of the $\overline{2} \overline{2} 0$ and 220 beams.

Note that the simulation program successfully tracked the changes in the image as the crystal thickness grew from the WPO domain of $35 \AA$, all the way to a thickness of $300 \AA$.

"Electron microscope image simulation", M.A. O'Keefe and P.R. Buseck, to be published. 
Simulated images of Vesuvianite in [001] orientation

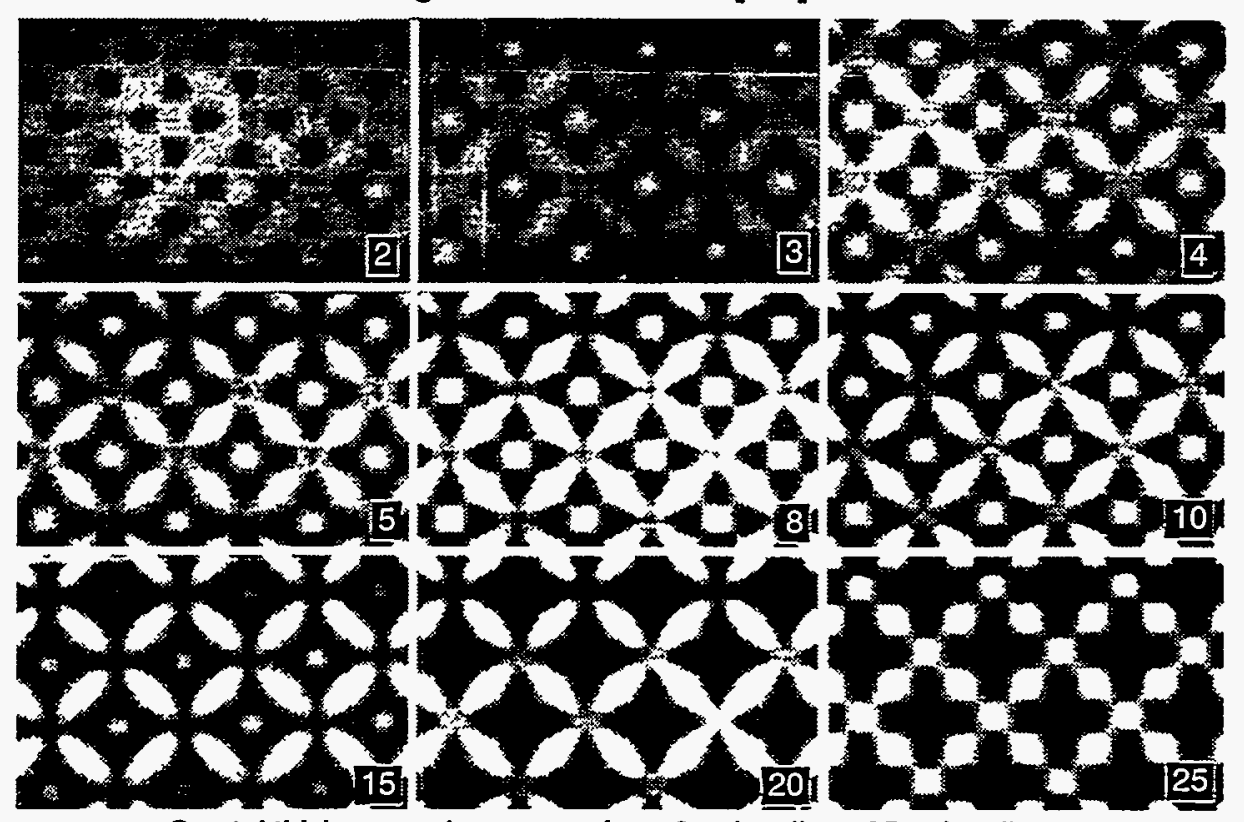

Crystal thickness values range from 2 unit cells to 25 unit cells

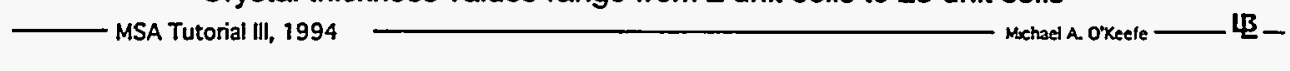

The figure shows simulated SHRLI images as crystal thickness is increased from the WPO domain to $300 \AA$ thickness.

The image character changes from WPO to "lattice with crosses with small center holes" to "lattice with crosses with no center holes" to "stubby-armed crosses". These changes are due to the thickness effects of complex non-WPO weightings of the four contributing image components $A$ through $D$.

In addition, the small white dots visible in the arms of the crosses in the thickest image are due to second-order interferences producing a contributing I image component of twice the frequency (half the spacing) of the strong $\mathrm{C}$ image component. 


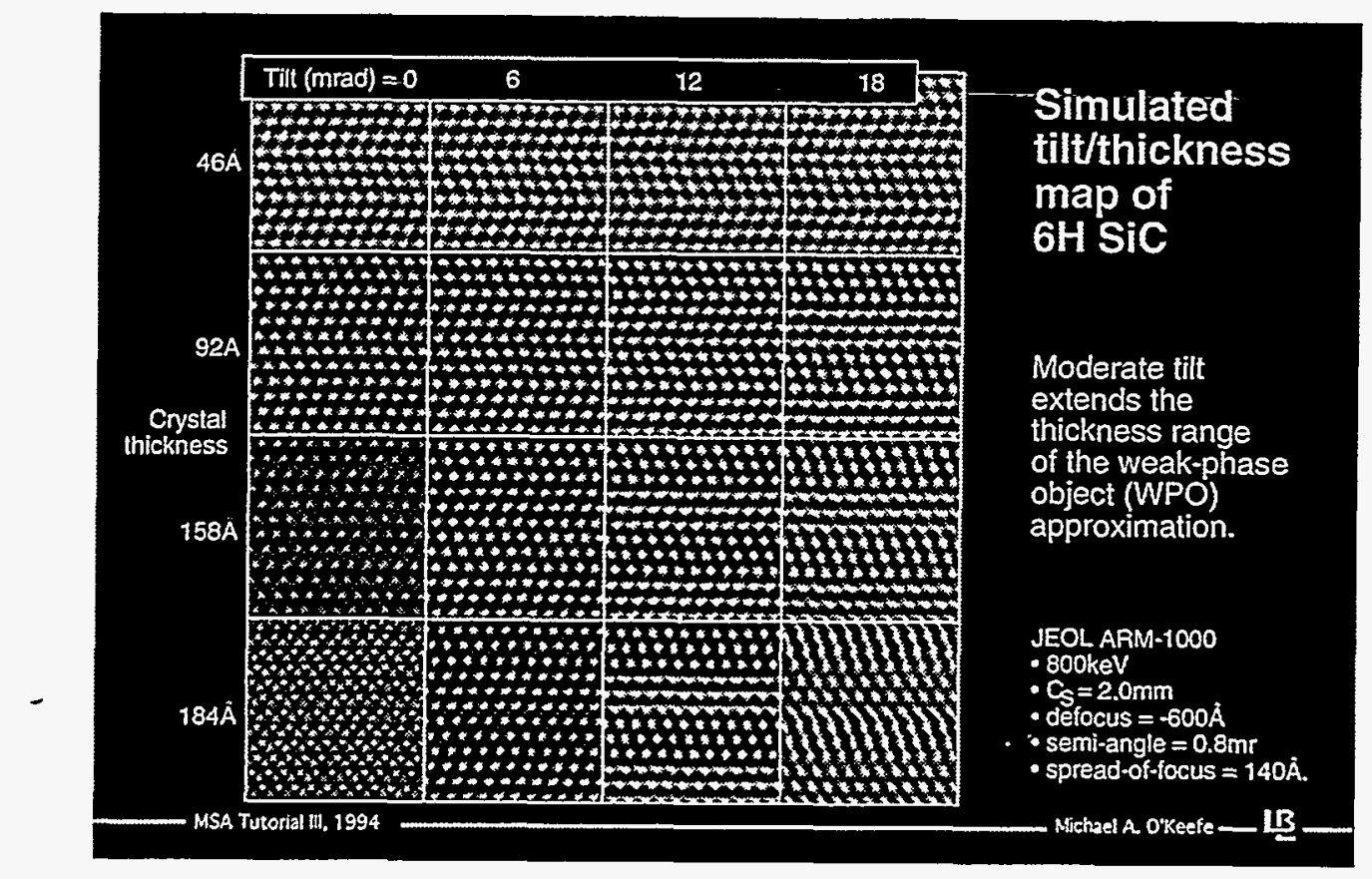

Extending the thickness range of WPO -- The crystal thickness to which the WPO approximation is valid may be extended by slight tilts of the specimen from the exact zone-axis orientation. These simulated images illustrate the effects of crystal tilt (up to 18 milliradian) on images of $6 \mathrm{H}-\mathrm{SiC}$ as a function of specimen thickness. The images show that a WPO-type of image can be obtained at $200 \AA$ thickness when the specimen is tilted 6 milliradian $\left(1 / 3^{\circ}\right)$ off the [110] zone axis, but only to $100 \AA$ when orientation is exact. Degradation in image symmetry limits the range of the thickness-extension effect in both thickness and tilt.

We can conclude that -

- the "best-looking" image does not guarantee that the specimen tilt is correct.

- for specimens slightly too thick to qualify as WPOs, slight tilt can be advantageous in order to simplify interpretation.

"Extending the "thin-crystal" condition by small crystal tilts -- why HREM images of SiC polytypes always look tilted", Michael A. O'Keefe and Velimir Radmilovic, in 50th Ann Proc. EMSA, Boston, Massachusetts (1992) 116-117. 


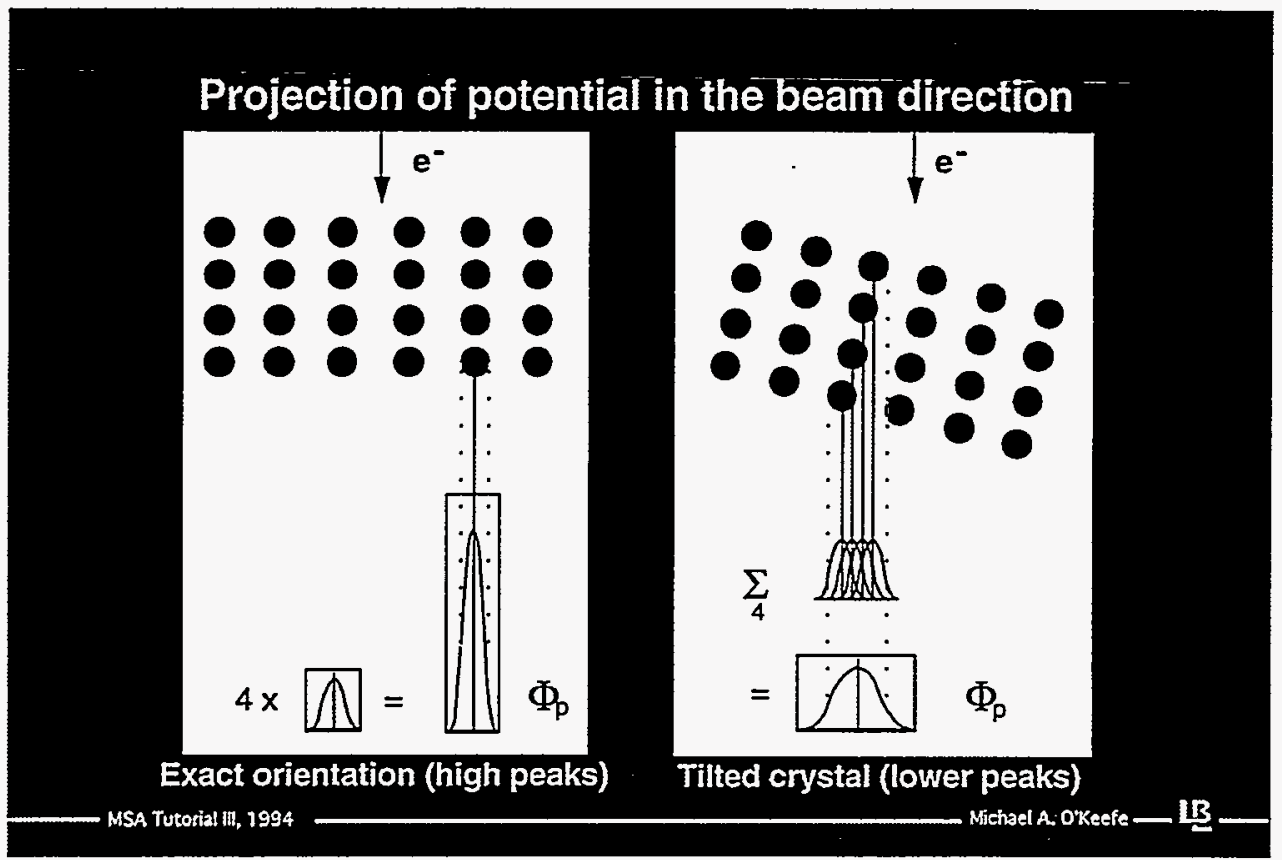

- Why does a small amount of crystal tilt make the crystal diffract like a much thinner crystal?.

- Because electrons scatter from the crystal potential, and any crystal tilt away from the zone-axis reduces the height of any potential peaks that would be projected in the direction of the incident beam.

- The diagram illustrates the effects of summing atom potentials. In the untilted case, individual atom potentials are exactly superimposed (left), maximizing the potential peak in the direction of the incident beam. In the tilted case, the atoms no longer line up exactly along the beam direction (right), producing a much lower peak in the incident beam direction.

- For the tilted crystal, the potential peak is much lower, and hence electron scattering is much less dynamic, making the resultant image appear to come from a thinner crystal.. 


\section{The weak-phase object (WPO) approximation}

Exit-surface wave under wpo conditions (weak scattering) -

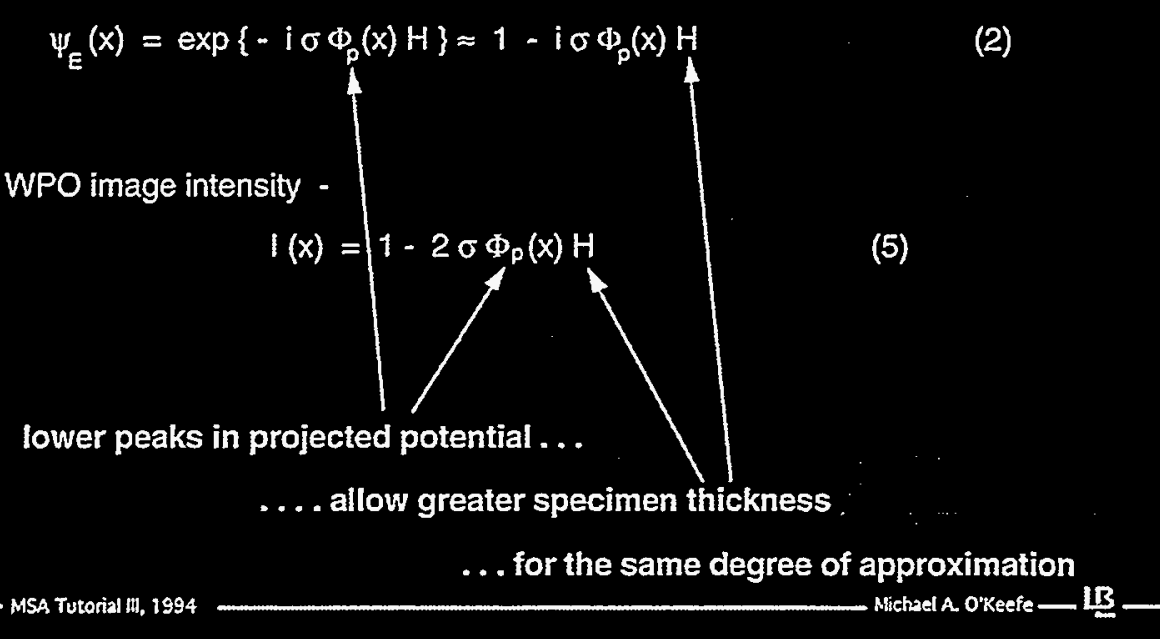

- Any weakening of the projected potential peak extends the thickness to which the weak-phase object approximation holds.

- When the product of the potential and the crystal thickness is low enough, the exponential describing electron scattering can be approximated as a difference (equation1).

- Then the image intensity can be approximated by the WPO expression (equation 2).

- The WPO image intensity approximation gives the same result as long as the product of the potential and the crystal thickness remains the same. If the potential maximum is halved, then the crystal thickness can be doubled.

- Tilting the crystal lowers the potential and increases the thickness to which the WPO approximation holds. 




Tilt makes crystals produce "thinner" images -

Simulated images of aluminum in [001] orientation display less dynamical characteristics when the specimen crystal is tilted; the proportion of second-order contributions is lower, and the image appears to come from a thinner crystal.

The figure illustrates this effect for $80 \AA \dot{A}$ thickness and tilts up to 16 milli-radian. Interestingly, even though the potential is quite smeared out at the higher tilts, the images appear much less so. Simulations show no significant effect for the 1 milliradian tilt (the peak potential drops from 106 volts to 99 volts); this means that normal beam convergences (usually near 1 milliradian) need not be included in the diffraction calculation (at least up to $80 \AA \dot{A}$ thickness for atoms as light as aluminum).

The figure shows projected potentials (left column), and simulated images for aluminum in [001] orientation. Each potential (left) is marked with tilt (upper left in milliradian) and potential peak (lower right in volts). Increasing tilt smears out the projected crystal potential (left column) and lowers the peak from the untilted maximum of 106 volts to 45 volts. Simulations are for $400 \mathrm{keV}, \mathrm{Cs}=1 \mathrm{~mm}$, convergence semi-angle of $1.0 \mathrm{mrad}$ (i.e. gaussian standard deviation of 0.77 milliradian), spread of focus of $60 \dot{A}$ standard deviation, and defocus values (marked) from $-350 \hat{A}$ to $-650 \AA$ in steps of $-50 \dot{A}$. The optimum defocus image ("black-atom" image) is near the center of the defocus range. - Non-linear second-order contributions produce white dots in the centers of the blacks when the specimen is untilted (top); this effect decreases with increasing tilt.

"The effects of small crystal tilts on dynamical scattering: why simulated images are thinner than experimental ones", Michael A. O'Keefe and Velimer Radmilovic, in 51st Ann. Proc. MSA, Cincinatti, Ohio (1993) 980-981. 


\section{- Outline -}

What use is HRTEM image simulation?

History -- image simulation was conceived for structure determination.

Uses - the uses of image simulation and image processing -- structure determination is only one of five uses for image simulation.

Structure determination -- recipes and examples of structure determination by matching of experimental images and simulated ones.

How does HRTEM image simulation work?

WPO -- interpreting images with the weak phase-object approximation; Why do some images "look like" the structure?

- Bevond WPO -- the steps involved in image simulation; modelling the high-resolution TEM in the computer; computing dynamical diffraction; computing lens effects.

Using HRTEM image simulation

Running simulations -- obtaining values of simulation parameters. Handling defects -- what additional precautions are necessary?

\section{Theory-}

- Beyond WPO. WPO is fine for thin-crystalScherzer-defocus images, but what about images where dynamical diffraction and non-linear interferences matter? What parameters need to be known to use the full power of image simulation? What limits exist? 


\section{Beyond the weak phase-object approximation}

Simulation programs that accurately model the HRTEM go beyond the linear approximations used in deriving the WPO approximation --

- kinematical (single) scattering reverts to dynamical (multiple) scattering

- WPO lens reverts to frequency-dispersive lens --

$$
\chi(u)=-\pi / 2(\sin \chi=-1) \quad \text { reverts to } \quad \chi(u)=\pi \varepsilon \lambda u^{2}+\pi C_{S} \lambda^{3} u^{4} / 2
$$

Topics --

- relation of simulation steps to the HRTEM imaging system

- computing dynamical scattering

- simulation parameters

- passbands and passband-resolution

- damping functions and transmission cross-coefficients

\section{"Proper" simulation of HRTEM Images -}

In order to model the image-formation process of the highresolution tranmission electron microscope, we need to discard the approximations we made in deriving the weak-phase-object approximation. Thus we model the electron scattering using a full dynamical approach instead of restricting it to a kinematical (thin-crystal) model. Similarly, the phase changes imposed on the diffracted beams by the objective lens are now a function of the spatial frequency corried by any particular beam, and not a constant value of $-\pi / 2$.

We will look at --

- the steps involved in modelling the HRTEM

- the multislice approach to modelling dynamic scattering

- parameters needed for simulations

- CTF passbands and their resolution

- the origin and effects of resolution-limiting damping functions on linear-image CTFs, and the extension to the more-general transmission cross-coefficients. 


\section{The WPO image's approximation to the CTF}

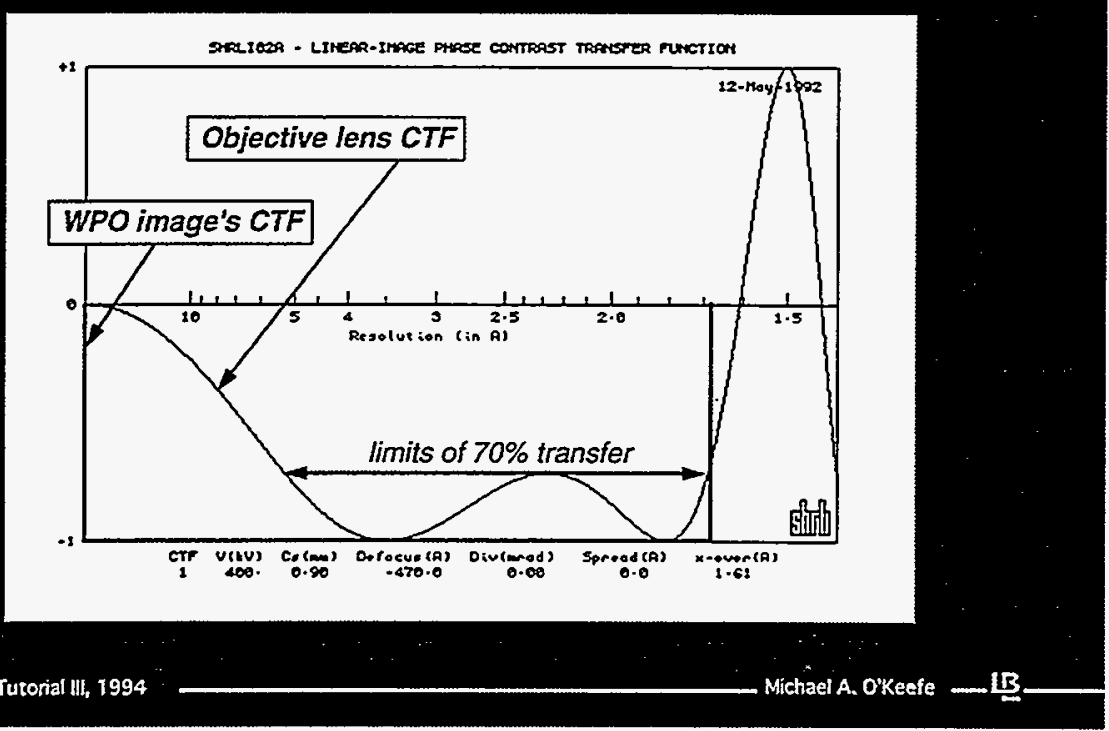

\section{The WPO image's approximation to the damped CTF}

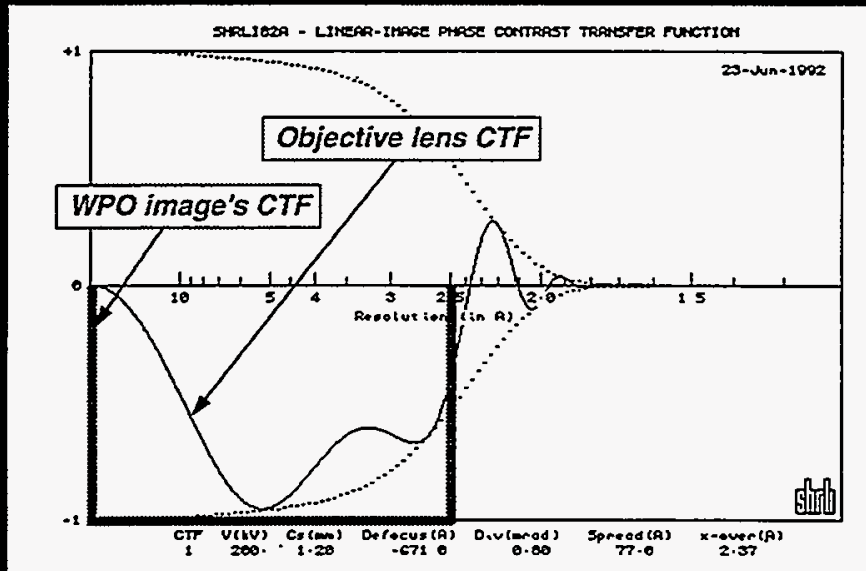

\section{The WPO image's approximate CTF}

- The thicker lines show the approximation assumed by choosing the value of $\sin \chi$ to be equal to minus one over the full frequency range of the CTF passband.

- Compared with the undamped CTF (upper slide), the CTF used in forming the WPO image is approximately correct. In reality, the effects of limited coherence (spread of focus and incident beam convergence) make the approximation even less precise (lower slide). 


\section{Relationship of image simulation steps and TEM functions}

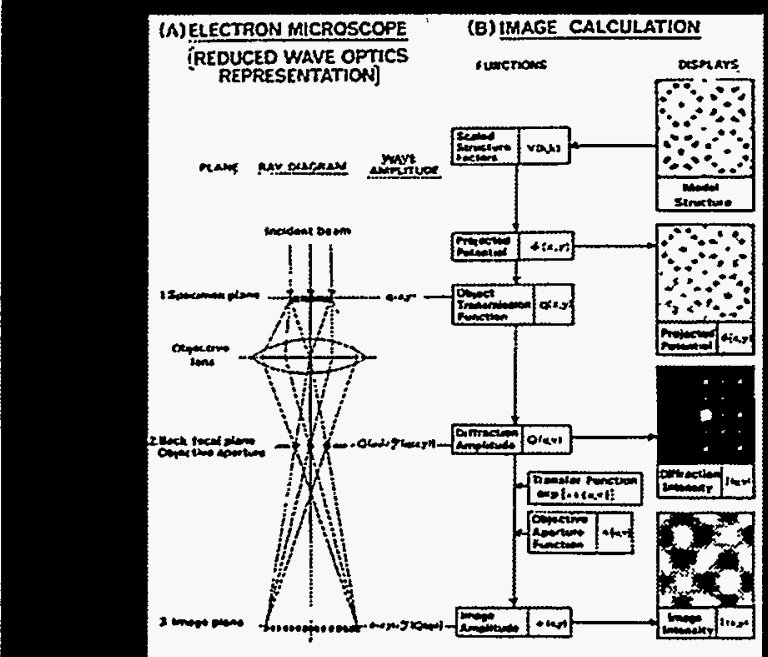

- The model structure is used to produce the projected polential $\phi(x, y)$.

- The projected potertiv: is used is produce the
cymanticallysyramticall satitered Exil-surtace

- The gix,y acts as the obres: for the the iars obas, ary the :arts ghase shote ate inposed of the cymponents
of cix,y.

- The inage is praduced by Foirier Iranstomalor of the gberrales o\{u,4\}.

This wave-optics view is of a "reduced" TEM. It shows the essential stages in the formation of the high-resolution image, and the equivalent steps in the image simulation program.

We have reduced the HRTEM to three items, an illuminating electron beam, a specimen, and an objective lens. We do not have to treat other lenses since they can be designed not to introduce modifications into the primary image that is formed by the objective lens. But the objective lens forms the primary image from diffracted beams leaving the exit surface of the specimen and must accept them at their Bragg angles, thus contributing spherical aberration.

Specimen We first compute the structure factors (or Fourier components of the crystal potential) for our model structure, and then produce the projected potential $\phi(x, y)$. We check $\phi(x, y)$ against a display of the model in the same projection. Then we use dynamical scattering theory (usually multislice) to compute the object transmission function and thus the exit-surface wave- function.

Optical system We FFT the exit-surface wave-function to get the diffracted beams as they appear in the back-focal plane of the lens, then impose the objective lens phase shifts to produce the Fourier components of the image amplitude spectrum. An auto-correlation of the image amplitude components produces the image intensity spectrum, and an IFT of the intensity spectrum yields the image intensity as it appears in the image plane of the lens.

Thus the simulation calculation has two major parts: (1) computing the dynamical scattering from the model object, and (2) computing the image intensity from the exit-surface wave. 

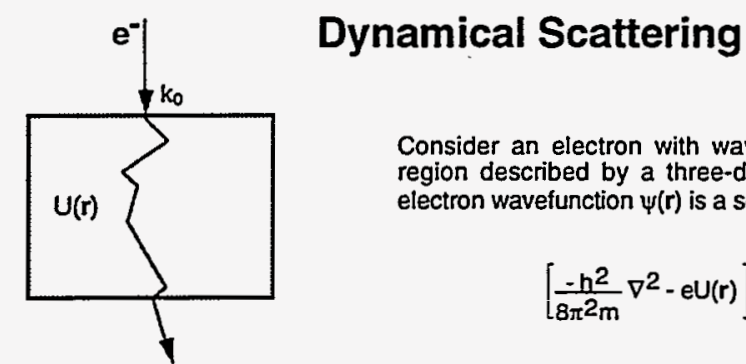

Consider an electron with wave-vector $k_{0}$ travelling through a region described by a three-dimensional potential $U(r)$. The electron wavefunction $\psi(\mathbf{r})$ is a solution to Schrödinger's equation

$$
\left[\frac{-h^{2}}{8 \pi^{2} m} \nabla^{2}-e U(r)\right] \psi(r)=\frac{h^{2} k_{0}^{2}}{2 m} \psi(r)
$$

\begin{tabular}{l|l}
$\begin{array}{l}\text { Electron energy } \\
\text { is much greater } \\
\text { than the energy } \\
\text { of the potential }\end{array}$ & $\mathrm{eU}(r) \ll \frac{h 2 k_{0}}{2 m} \rightarrow\left[\nabla^{2}+4 \pi i k_{0} \frac{\partial}{\partial z}+V(r)\right] \cdot \phi(r)=0$ \\
& where $V(r)=\frac{8 \pi^{2} m e}{h^{2}} U(r)$
\end{tabular}

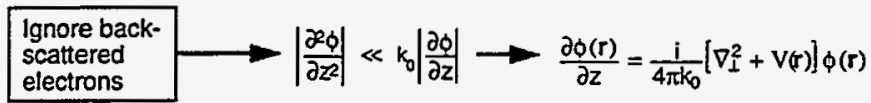

Solution has

propagation-

operator

$$
\phi(x, y, \varepsilon)=\exp \left(\frac{i}{4 \pi k_{0}}\left[\varepsilon \cdot \nabla_{1}^{2}+\int_{0}^{\varepsilon} v(r) \cdot d z\right]\right) \cdot \phi(x, y, 0)
$$

- MSA tutorial III, 1994

\section{Dynamical Scattering}

Consider an electron with wave-vector $k_{0}$ travelling through a region described by a three-dimensional potential $U(r)$. The electron wavefunction $\psi(r)$ is a solution to Schrödinger's equation -

$$
\left[\frac{-h^{2}}{8 \pi^{2} m} \nabla^{2}-e U(r)\right] \psi(r)=\frac{h^{2} k_{0}^{2}}{2 m} \psi(r)
$$

For high-energy electrons the energy of the incoming electron is much greater than that of the potential field, so $e U(r) \ll \frac{h 2 k_{0}^{2}}{2 m}$ and thus the potential $U(r)$ can be considered a perturbation, and the total wavefunction can be written as

$$
\psi(\mathbf{r})=\phi(\mathbf{r}) \cdot \exp \left\{2 \pi \mathrm{i} \mathbf{k}_{0} \cdot \mathbf{r}\right\}=\phi(\mathbf{r}) \cdot \exp \left\{2 \pi i k_{0} \cdot z\right\}
$$

Then

$$
\begin{gathered}
\text { Then } \\
{\left[\nabla^{2}+4 \pi i k_{0} \frac{\partial}{\partial z}+\frac{8 \pi^{2} m e}{h^{2}} U(r)\right] \cdot \phi(r)=0} \\
\text { Or }\left[\nabla^{2}+4 \pi i k_{0} \frac{\partial}{\partial z}+V(r)\right] \cdot \phi(r)=0 \quad \text { where } V(r)=\frac{8 \pi^{2} m e}{h^{2}} U(r)
\end{gathered}
$$

Ignoring back-scattered electrons is justified for $\left|\frac{\partial^{2} \phi}{\partial z^{2}}\right| \ll k_{0}\left|\frac{\partial \phi}{\partial z}\right|$ and leads to a first-order differential equation in $z$

$$
\frac{\partial \phi(r)}{\partial z}=\frac{i}{4 \pi k_{0}}\left[\nabla_{\perp}^{2}+V(r)\right] \phi(r)
$$

The solution to this equation can be written [1] as

$$
\phi(x, y, \varepsilon)=\exp \left(\frac{i}{4 \pi k_{0}}\left[\varepsilon \cdot \nabla_{\perp}^{2}+\int_{0}^{\varepsilon} V(r) \cdot d z\right]\right) \cdot \phi(x, y, 0)
$$

[1] R. Kilaas, M.A. O'Keefe and Kannan M. Krishnan "On the inclusion of upper Laue layers in computational methods in HRTEM", Ultramicroscopy21 (1987) 47-62. 


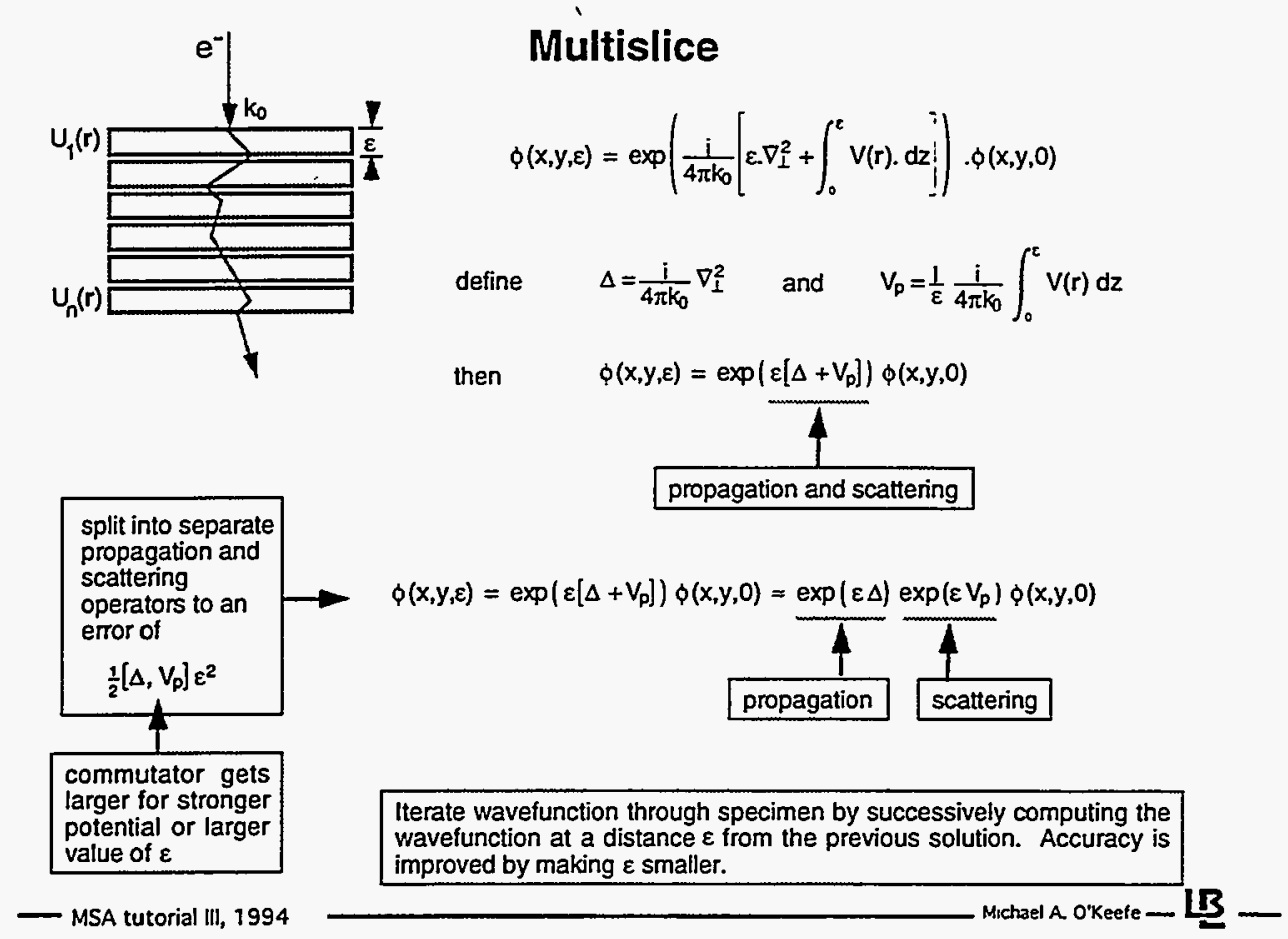

\section{The multislice approach}

Thus the electron wavefunction $\phi(x, y, \varepsilon)$, at a distance $z=\varepsilon$ from the position $z=0$, is given by operating on the wavefunction $\phi(x, y, 0)$ at $z=0$.

We can define $\quad \Delta=\frac{i}{4 \pi k_{0}} \nabla_{\perp}^{2}$ and $\quad V_{p}=\frac{1}{\varepsilon} \frac{i}{4 \pi k_{0}} \int_{0}^{\varepsilon} V(r) d z$

and write

$$
\phi(x, y, \varepsilon)=\exp \left(\varepsilon\left[\Delta+V_{p}\right]\right) \phi(x, y, 0)
$$

Physically, the operator $\exp \left(\varepsilon\left[\Delta+V_{p}\right]\right)$ represents the actions of propagation and scattering of the electron within the specimen. We are unable to apply both actions simultaneously, so we make the approximation of applying them consecutively [1]. We put

$$
\phi(x, y, \varepsilon)=\exp \left(\varepsilon\left[\Delta+V_{p}\right]\right) \phi(x, y, 0)=\exp (\varepsilon \Delta) \exp \left(\varepsilon V_{p}\right) \phi(x, y, 0)
$$

This approximation is correct to first order in $\varepsilon, \Delta$ and $V p$, with an error of the order of the commutator $\frac{1}{2}\left[\Delta, V_{p}\right] \varepsilon^{2}$, where $\varepsilon$ is the distance through which the wave has travelled in the $z$ direction. But it does allow us to compute the electron wave by first applying the scattering and then the propagation (or vice versa).

In order to compute $\phi(x, y, \varepsilon)$ from $\phi(x, y, 0)$ with sufficient accuracy we need to ensure that the value of the error commutator remains small. It turns out that $\varepsilon$ should be no more than a few $\AA$ for electrons of the energy used in HREM and for the potentials present in typical specimens. Thus to compute the dynamical scattering from a specimen thicker that this, we need to apply the calculation iteratively, i.e. we compute the wavefunction a few $\dot{A}$ into the specimen then use this value as the starting wavefunction to compute the wavefunction a further few $\dot{A}$ into the specimen; proceeding iteratively, we can compute the wavefunction at the exit surface of the specimen. Note that we can make the error as small as we like by making $\varepsilon$ sufficiently small.

To perform the multislice calculation, we need to consider the specimen as divided into "slices" with normals in the beam direction. We compute the propagation function $\Delta$ for each slice, and the projected potential within each slice (and from these the scattering functions $V p$ ). Then we propagate and diffract through each slice, to generate the wavefunction at the exit surface. Note that the error commutator becomes larger for scattering from structures containing a high density of heavy atoms, so $\varepsilon$ must be made smaller, requiring more slices in the dynamical scattering computation. Most simulation programs make this adjustment automatically.

[1] P. Goodman and A.F. Moodie, "Numerical evaluation of $n$-beam wavefunctions in electron scattering by the multi-slice method", Acta Cryst. A30 (1974) 280. 


\section{- Outline -}

What use is HRTEM image simulation?

History -- image simulation was conceived for structure determination.

Uses -- the uses of image simulation and image processing -- structure determination is only one of five uses for image simulation.

Structure determination - recipes and examples of structure determination by matching of experimental images and simulated ones.

How does HRTEM image simulation work?

WPO -- interpreting images with the weak phase-object approximation; why do some images "look like" the structure?

Bevond WPO -- the steps involved in image simulation; modelling the high-resolution TEM in the computer; computing dynamical diffraction; computing lens effects.

Using HRTEM image simulation

- Running simulations - obtaining values of simulation parameters. Handling defects -- what additional precautions are necessary?

\section{Using Image Simulation-}

\section{- Running. How do we run the programs?}

- How do we find parameter values to input? 


\section{Image simulation parameters}

The model structure

- unit cell parameters -- $a, b, c, \alpha, \beta, \gamma$

- space group number, or list of symmetry operators

- atom data -- $x, y, z$, symbol/atomic number, occupancy, D-W factor

\section{The specimen parameters}

- specimen orientation -- zone axis, tilt (center of Laue circle)

- specimen thickness

\section{The microscope beam parameters}

- accelerating voltage

- misalignment - center of optic axis (relative to 000 beam)

- convergence -- semi-angle at specimen

- coherence -- energy spread, high-voltage ripple

The microscope lens parameters
objective aperture - size and position (relative to 000 beam)
- lens defocus
- lens aberrations - spherical aberration $\left(\mathrm{C}_{\mathrm{s}}\right)$, chromatic aberration $\left(\mathrm{C}_{\mathrm{C}}\right)$
coherence -- objective lens current ripple
- vibration -- of the specimen within the lens, or the image on the film
Display parameters
- image visibility -- contrast, brightness
- image size -- magnification/zoom-factor, number of unit cells

Simulation of a high-resolution TEM image requires modelling both the specimen and the microscope in the computer. Simulation programs require the parameters listed in order to carry out the modelling process.

Model structure -- unit cell parameters are available from the literature, or from the designer of a "defect cell". For a perfect cell, a standard space group can be used to generate a list of atom positions from a basis; for defects cells, a list of symmetry operators can often be used. For each atom in the structure, we need its threedimensional position within the unit cell ( $x, y, z$ in fractional coordinates), an ID (either chemical symbol or atomic number), the site occupancy (usually 1.0), and Debye-Waller factor to describe any smearing due to temperature.

Specimen -- orientation is input as a zone axis (e.g. [110]), and any tilt away from this axis is input in terms of the Laue circle center coordinates (in the Takeuchiite example the coordinates were 7,7 ). Sample thickness is expressed either as $\dot{A}$ (or $\mathrm{nm}$ ), or in terms of number of unit cells or number of slices.

Microscope beam -- the programs need the accelerating voltage to compute wavelength and interaction parameter (scattering cross-section); usually the nominal voltage is good enough (e.g. 200kV). Any misalignment of the beam from the optic axis is input as the position of the optic axis on the diffraction pattern (try zero initially). Convergence (usually as the semi-angle in milliradians) can be measured from a focussed diffraction pattern [1,2]. Energy spread is more difficult -- start at $1.0 \mathrm{eV}$ for an $\mathrm{LaB} 6$ gun and $0.3 \mathrm{eV}$ for a FEG. High-voltage ripple is of the order of 1ppm.

Lens parameters -- size and position of the objective aperture can be measured from a diffraction pattern [1,2]. Size is usually input in terms of $\AA$ or reciprocal $\dot{A}$; position is input as the coordinates of the aperture center on the diffraction pattern. Lens defocus may be calibrated in terms of number of clicks from a standard defocus such as minimum or optimum (usually maximum contrast). Spherical aberration can be measured; often the nominal figure is good enough. The nominal chromatic aberration figure is used with beam energy spread ( $\delta E$ ), high-voltage ripple $(\delta V)$ and lens-current ripple $(\delta)$ to compute a nominal spread-of-focus $(\Delta)$ for input to the simulation program., where $\Delta=C c . \sqrt{ }\left\{(\delta E / E)^{2}+(\delta V / N)^{2}+(2 \delta / /)^{2}\right\} ; \Delta$ is often adjusted from this value based on experience. Note that $\delta E$ depends on filament condition and bias. Vertical vibration of the specimen adds to the spread of focus; horizontal vibration is a separate parameter and smears the image directly -- it may be as high as $0.5 \AA$.

Display parameters -- the simulation programs provide parameters to control the brightness and contrast of the image; these factors may be adjusted for each individual image or held constant for comparison of images within a focal or thickness series. Size and area of the image can be specified.

[1] "n-beam lattice images, Vl. Degradation of image resolution by a combination of incident-beam divergence and spherical aberration", M.A. O'Keefe and J.V. Sanders, Acta Cryst. A31 (1975) 307-310.

[2] "Electron image simulation; a complementary processing technique", M.A. O'Keefe in Proc. 3rd Pfefferkorn Conf. on Electron Optical Systems, Ocean City, Maryland (1984) 209-220.

[3] "Determination of defocus values using 'Fourier images' for high resolution electron microscopy", S. lijima and M.A. O'Keefe, J. Microscopy 117 (1979) 347-354. 
Table of HRTEMs with point-to-point resolution better than $2.5 \AA^{a)}$

\begin{tabular}{|c|c|c|c|c|c|}
\hline \multirow[b]{2}{*}{ Microscope } & \multirow{2}{*}{$\begin{array}{l}\text { Maximum } \\
\text { operating } \\
\text { energy } \\
(\mathrm{keV})\end{array}$} & \multirow{2}{*}{$\begin{array}{c}\mathrm{C}_{\mathrm{s}} \\
(\mathrm{mm})\end{array}$} & \multirow{2}{*}{$\begin{array}{c}\mathrm{C}_{\mathrm{c}} \\
(\mathrm{mm})\end{array}$} & \multicolumn{2}{|c|}{$\begin{array}{l}\text { Theoretical resolutions }{ }^{\mathrm{b})} \\
(\AA)\end{array}$} \\
\hline & & & & Scherzerc) & Information Limit ${ }^{\mathrm{d}}$ ) \\
\hline Cambridge HREM & 600 & 2.5 & 2.7 & 1.70 & 1.22 \\
\hline Hitachi H8100 & 200 & 0.7 & 1.1 & 2.07 & 1.54 \\
\hline Hitachi HF2000e) & 200 & 1.2 & 1.5 & 2.37 & 1.26 \\
\hline Hitachi HF2000-UHR ${ }^{\mathrm{e})}$ & 200 & 0.7 & 1.0 & 2.07 & 1.03 \\
\hline Hitachi H9000-UHR & 300 & 0.9 & 1.5 & 1.84 & 1.36 \\
\hline Hitachi H9000-NAR & 300 & 0.68 & 1.4 & 1.71 & 1.32 \\
\hline Hitachi $300 \mathrm{keV} \mathrm{FEG}^{\mathrm{e}}$ ) & 300 & 0.9 & 1.5 & 1.84 & 1.07 \\
\hline Hitachi HU1250 & 1250 & 2.5 & 3.5 & 1.13 & 0.98 \\
\hline Hitachi H-1500 & 1300 & 1.85 & 3.4 & 1.03 & 0.95 \\
\hline ISI $^{f)} 002 \mathrm{~A}$ & 120 & 0.3 & 0.6 & 2.08 & 1.65 \\
\hline $\mathrm{ISI}^{\mathrm{f}} 002 \mathrm{~B}$ & 200 & 0.4 & 0.8 & 1.80 & 1.31 \\
\hline JEOL 2000EX & 200 & 0.9 & 1.2 & 2.21 & 1.61 \\
\hline JEOL 2010HT & 200 & 1.0 & 1.4 & 2.26 & 1.74 \\
\hline JEOL 2010UHR & 200 & 0.5 & 1.0 & 1.90 & 1.47 \\
\hline JEOL 2010Fe) & 200 & 0.5 & 1.0 & 1.90 & 1.03 \\
\hline JEOL 3010HT & 300 & 1.4 & 2.2 & 2.05 & 1.65 \\
\hline JEOL 3010UHR & 300 & 0.6 & 1.4 & 1.66 & 1.32 \\
\hline JEOL 4000EX & 400 & 0.9 & 1.65 & 1.61 & 1.20 \\
\hline JEOL HAREM & 500 & 1.0 & 1.4 & 1.48 & 0.97 \\
\hline JEOL ARM-1000 & 1000 & 2.3 & 3.4 & 1.26 & 1.07 \\
\hline JEOL Kyoto-1000 & 1000 & 1.7 & 3.6 & 1.17 & 1.10 \\
\hline JEOL ARM-1250 & 1250 & 1.6 & 4.0 & 1.02 & 1.05 \\
\hline Philips CM20/ST & 200 & 1.2 & 1.2 & 2.37 & 1.61 \\
\hline Philips CM200FEG/ST ${ }^{\mathrm{e}}$ & e) 200 & 1.2 & 1.2 & 2.37 & 1.13 \\
\hline Philips CM20/UT & 200 & 0.5 & 1.0 & 1.90 & 1.47 \\
\hline Philips CM200FEG/UT ${ }^{\mathrm{e}}$ & e) 200 & 0.5 & 1.0 & 1.90 & 1.03 \\
\hline Philips CM30/T & 300 & 2.0 & 2.0 & 2.25 & 1.58 \\
\hline Philips CM300FEG/T & 300 & 2.0 & 2.0 & 2.25 & 1.23 \\
\hline Philips CM30/ST & 300 & 1.2 & 1.5 & 1.98 & 1.36 \\
\hline Philips CM300FEG/STe) & e) 300 & 1.2 & 1.5 & 1.98 & 1.07 \\
\hline Philips CM300FEG/UT ${ }^{\mathrm{e})}$ & e) 300 & 0.7 & 1.5 & 1.73 & 1.07 \\
\hline
\end{tabular}

a) From M.A. O'Keefe, Ultramicroscopy, 47 (1992) 282-297.

b) Resolution figures for comparison only -- actual experimental resolution may be different.

c) CTF crossover at a defocus of 1.225 Scherzers.

d) Value of $1 /\left.1 \mathrm{k}\right|_{\Delta}$ at which the $\mathrm{E}_{\Delta}(\mathrm{k})$ envelope drops to $\exp (-2)$, assuming $1 \mathrm{ppm}$ ripple in lens and high-voltage power supplies, zero vibration, and energy spread of $1 \mathrm{eV}\left(\mathrm{LaB}_{6}\right)$ or $0.3 \mathrm{eV}$ (FEG).

e) Equipped with field emission gun (FEG)

f) Now Topcon 


\section{Image simulation parameters -- convergence}
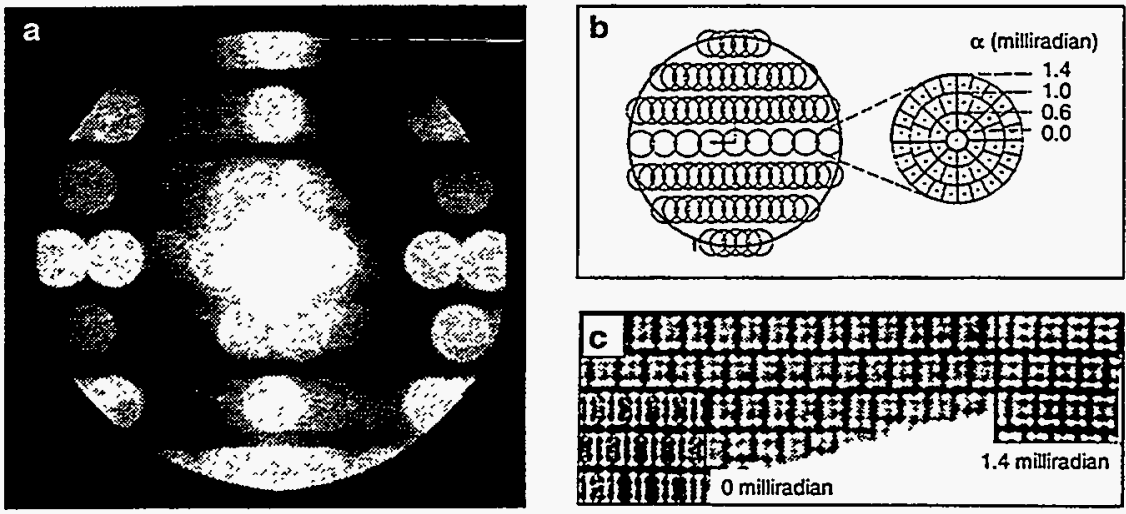

a. A diffraction pattem, focussed for imaging, provides a good approximation to the convergence parameter.

b. The convergence cone can be sampled and an image computed at each sampling point (i.e. each beam-misalignment angle).

c. Summation of the images includes the convergence effect, changing the simulation from non-matching (left simulated image) to matching (right).

- MSA tutorial III, 1994 Michael A. O'Keefe - W

Incident beam convergence -- the programs need the convergence semi-angle to compute its smearing (resolution-damping) effect on the image. A good estimate of its value can be obtained from a diffraction pattern recorded with the beam focussed as for imaging (here the inclusion of the objective aperture enables us to measure its radius accurately as well. The action of convergence is like summing over a bundle of incident beams misaligned from the optic axis.

The figure shows a method of including the effects of incident beam convergence by real-space summation. The degree of convergence is estimated by measuring the spot size in the experimental diffraction pattern (a). Each spot in the diffraction pattern is sub-sampled (b), and a series of images is computed at incident beam angles appropriately sampling the convergence cone. The images are added to produce the final result (c).

In this figure, (a) is an experimental diffraction pattern of $\mathrm{Nb12O29}$ with illumination set for imaging, showing the degree of incident beam convergence and the objective aperture size. (b) is a model of (a) showing the 49 sampling angles used to model each spot in the SHRLI programs. (c) shows that a simulation without summation (left) does not match the experimental image, whereas one with summation over the full 49 angles does (right). [1].

In reciprocal space, an approximately equivalent result can be obtained by modelling each diffraction spot with a top-hat function of appropriate size; then the TCC result matches real-space summation results (and also experiment).

[1] " $n$-beam lattice images, VI. Degradation of image resolution by a combination of incident-beam divergence and spherical aberration", M.A. O'Keefe and J.V. Sanders, Acta Cryst. A31 (1975) 307-310. 


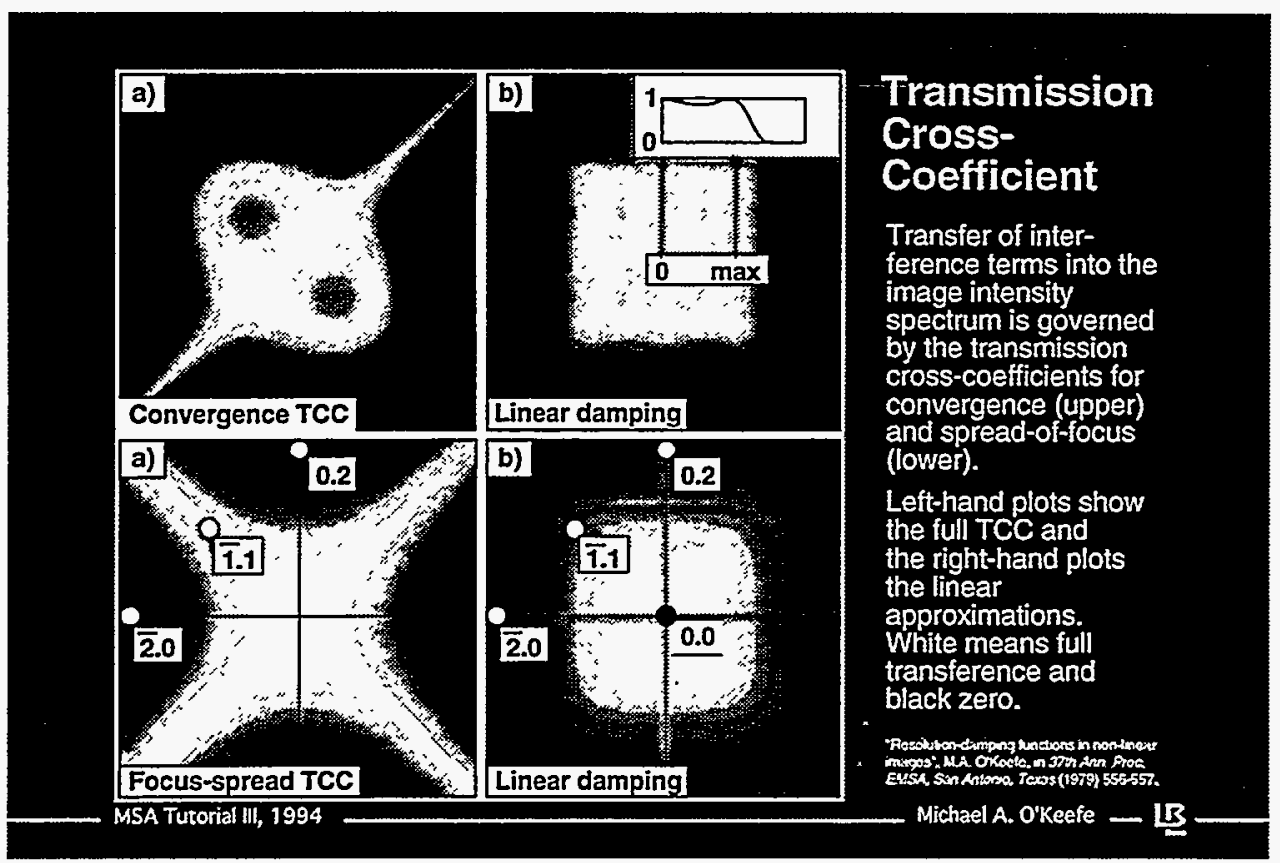

In a full image simulation, instead of including incident-beam convergence by a sum over images in real space, most simulation programs usually use the transmission cross-coefficient (TCC) to compute the damping as each interference pair transfers into the image intensity spectrum. Plots of the TCCs for convergence (upper) and spread of focus (lower) are shown above with white representing high transferrence and black for strong damping. The damping for any interference pair is found by reading across and up from the centers of the plots. Linear interferences (ones including the 000 beam) lie on the horizontal and vertical axes of the plots, thus the intensity profile along these directions is the same as the linear damping functions for the linear-image CTFs (e.g. insert at top right).

The convergence TCC (upper) varies with defocus; the plot shown is for Scherzer defocus. The spread-of- focus TCC (lower) does not vary with defocus.

Note that the spread-of-focus TCC (lower left) has a zero damping effect on interferences between the nth and -nth diffracted beams, so that spatial frequencies of $\mathbf{2 n}$ will be present in the image intensity spectrum (and visible in the image) if the $n$th beam is strong enough to produce a -non interference term of amplitude comparable to the n 0 term. This effect can be seen in the thickest vesuvianite image (300A) where the 440 frequencies, generated by "cross-aperture" interferences of the 220 beams, produce small white "holes" in the arms of the black crosses. 


\section{Scherzer Resolution for the Microscope}

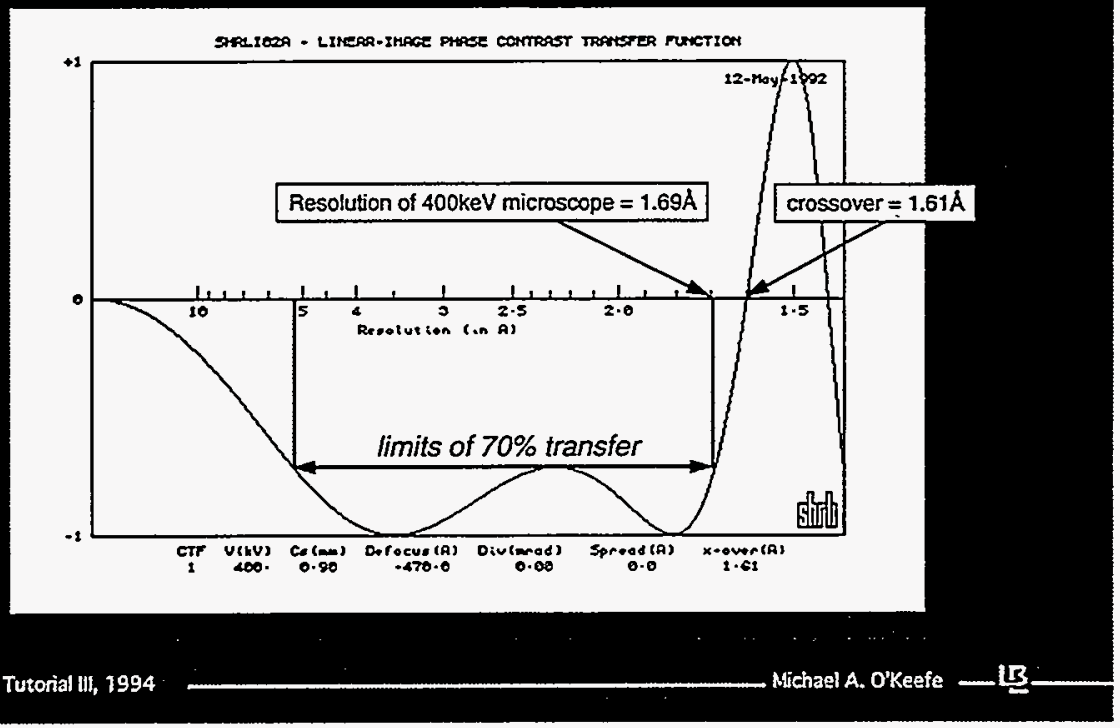

\section{MICROSCOPE RESOLUTION -- SCHERZER}

- a CTF for a 400keV HRTEM at "optimum" or "Scherzer" defocus, i.e. a defocus of $\sqrt{ } 1.5$ scherzers. A common definition of the Scherzer resolution is the $70 \%$ limit of transfer. Or sometimes at crossover.

- Whichever we agree to use, it defines the Scherzer resolution of the microscope -- in this case, better than $1.7 \AA$. 


\section{Spacings beyond the Scherzer passband can be transferred}

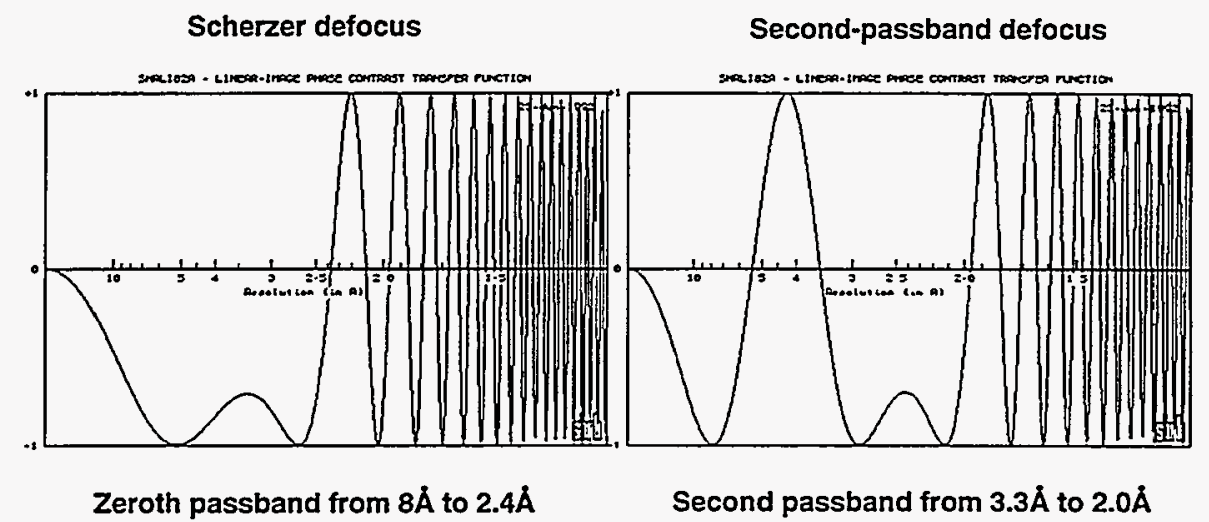

JEOL $200 C X$ with spherical aberration coefficient of $1.2 \mathrm{~mm}$ at $200 \mathrm{keV}$

- MSA Tutorial Ill, 1994 Michael A. O'Keefe - Щ-

\section{ZEROTH AND SECOND PASSBANDS}

- By using several images, we can get at more information. For a $200 \mathrm{keV}$ high-resolution electron microscope with an objective lens Cs of $1.2 \mathrm{~mm}$, the second- passband image contains frequencies correctly transferred over the range from $3.3 \AA$ to $2.0 \AA$. The Scherzer-defocus (zero-passband) image has correctly-transferred frequencies on the range from $8.0 \AA$ to $2.4 \AA$. In this case, two images cover a spatial frequency range from $8 \AA$ to $2 \AA$.

- The range from $8 \AA \AA$ to $2 \AA$ can be exploited by combining images by image processing or series reconstruction.

- Alternatively, matching with images generated from a model by image simulation will test the model over the full range from $8 \AA \AA$ to $2 \AA$.

- Note that the major passbands occur at defocus values of $\sqrt{ }[(4 n+3) / 2]$ scherzers, where $n$ is the number of the passband and one scherzer is defined as $-\sqrt{ }[\mathrm{Cs} \lambda]$. Thus Scherzer defocus occurs at $\sqrt{ } 1.5$ scherzers, first passband defocus at $\sqrt{3} .5$ scherzers, and second passband defocus at $\sqrt{5} .5$ scherzers. To determine the number of the passband defocus from a CTF plot, count the number of zero-crossings before (to the left of) the main passband. 


\section{Damping functions for Linear Images}

Spread of Focus Damping Function:

$$
E_{\Delta}(k)=\exp \left(\frac{1}{2} \pi^{2} \lambda^{2} \Delta^{2} \mathbf{k}^{4}\right) \quad|k|=\frac{1}{\sqrt{\pi \lambda \Delta / 2}}
$$

Beam Convergence Damping Function:

$$
\begin{gathered}
E_{\alpha}(\mathbf{k})=\exp \left\{-\pi^{2} \alpha^{2}\left(\varepsilon+\lambda^{2} C_{s}^{2} k^{2}\right)^{2} k^{2}\right\} \\
|\mathbf{k}|_{\alpha}=S_{+}^{1 / 3}+S_{-}^{1 / 3}
\end{gathered}
$$

where $S_{ \pm}=\left[\frac{3.3}{4 \pi \alpha} \pm \sqrt{\frac{\varepsilon^{3}}{27 C_{S} \lambda^{2}}+\left(\frac{3.3}{4 \pi \alpha}\right)^{2}}\right] / C_{S} \lambda^{2}$

- MSA Tutorial III, 1994 Michzel A. OKeefe - L B

\section{DAMPING FUNCTIONS}

- There exist limits to how far we can use defocus to get at the information in higher spatial frequencies.

- In any image, two effects are damping the transfer of information from the image amplitude spectrum into the image intensity spectrum. Under linear (thin-crystal) conditions, they impose damping envelopes on the CTF. The effects are --

(1) Spread of focus

(2) Incident beam convergence

- We can write down expressions for the damping envelope functions. In the linear case they are --

(1) $E_{\Delta}(k)$ due to the spread of focus formed from energy spread (and lens and high-voltage fluctuations) by the chromatic aberration coefficient $\mathrm{C}_{\mathrm{C}}$.

(2) $E_{\alpha}(k)$ due to convergence of the incident electron beam.

- We can define cutoffs $|\mathbf{k}|_{\Delta}$ and $|\mathbf{k}|_{\alpha}$ for the two damping functions at the values of spatial frequency beyond which transfer is negligible -- usually taken to be the spatial frequency at which they fall to $\exp (-2)$. 


\section{Modelling the convergence effect with top-hat \& Gaussian}

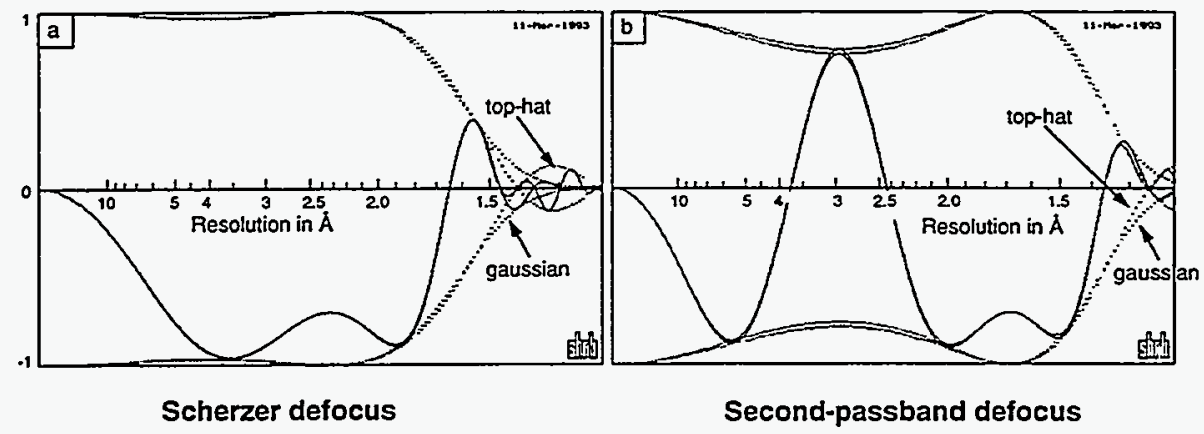

Phase-contrast transfer functions (solid lines) and convergence damping functions (dotted) for the gaussian and top-hat models of the incident beam convergence. The curves match (except that the gaussian envelope falls smoothly to the axis, whereas the top-hat envelope crosses it) when the proportionality factor is set to 0.77 .

$400 \mathrm{keV}, \mathrm{Cs}=1.0 \mathrm{~mm}$, defocus values of (a) $\sqrt{1.5}$ scherzers, and (b) $\sqrt{5.5}$ scherzers.

$\alpha=1.0$ milliradian.

- MSA Tutorial III, 1994

Michael A O'Keefe - LB

\section{Incident-beam-convergence modelling -- .}

For linear images (from specimens thin enough to behave as weak phase objects), the transmission cross-coefficient (TCC) can be simplified and described in terms of "damping envelope" functions that multiply the usual phase-contrast transfer function. The envelope for a top-hat convergence model crosses the axis near its cutoff frequency. A further approximation is to use a gaussian model for the diffraction spot intensity, giving an envelope that asymptotes smoothly to the axis. In the case of the top-hat model, the radius of the top-hat is obviously correct when set to the convergence semi-angle; for the gaussian model the standard deviation of the gaussian is set proportional to the convergence semi-angle. The proportionality constant is important, especially since many simulation programs use a gaussian model to form the TCC used in simulating images. Comparisons of plots of top-hat and gaussian damping. functions show that they match when the standard deviation of the gaussian is set to 0.77 times the convergence semi-angle.

The figure shows phase-contrast transfer functions (solid lines) and convergence damping functions (dotted) for the gaussian and top-hat models. The curves match (except that the gaussian envelope falls smoothly to the axis, whereas the top-hat envelope crosses it) when the proportionality factor is set to 0.77 . Plots are for $400 \mathrm{keV}, \mathrm{Cs}=1.0 \mathrm{~mm}$, semi-angle $=1.0$ milliradian, and defocus values of (a) $\sqrt{1} .5$ scherzers, and (b) $\sqrt{5} .5$ scherzers.

[1] "Using convergence and spread-of-focus parameters to model spatial and temporal coherence in HRTEM image simulations", Jan-Olle Malm and Michael A. O'Keefe, Proceedings 51st MSA (1993) 974-975. 


\section{Modelling the coherence effects with Gaussians}

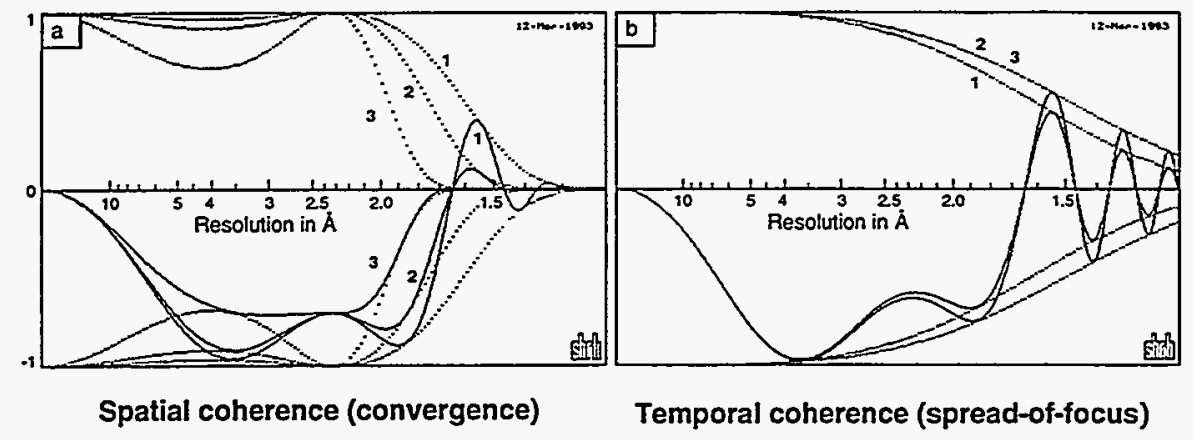

Phase-contrast transfer functions (solid lines) and convergence damping functions (dotted) for gaussian models of the incident beam convergence of 1 milliradian (a) and spread-offocus of $60 \AA$ (b).

(1) SHRLI result using a proportionality factor of 0.77 for convergence.

(2) "Classic" result.

(3) EMS result.

Plots for $400 \mathrm{keV}, \mathrm{Cs}=1.0 \mathrm{~mm}$, and defocus value of $\sqrt{1} .5$ scherzers.

- MSA Tutorial III, 1994 Mchael A O'Keefe - 4 -

Phase-contrast transfer functions and damping functions for (a) gaussian model of convergence at alpha=1.0 milliradian, (b) gaussian model of spread of focus at delta $=60 \AA$. Numbers on the curves refer to expressions used in the references. Plots for $400 \mathrm{keV}, \mathrm{Cs}=1.0 \mathrm{~mm}$, and defocus value of $\sqrt{1.5}$ scherzers.

Several "classic" expressions for gaussian convergence damping envelopes exist, but none of these appears to have been tested against the top-hat result. Spence[2] and Reimer[2] use a gaussian with alpha set to the half-width at half maximum (HWHM), Krivanek[2] uses the spot diameter (twice alpha) as the FWHM, and Stadelmann[3] chooses half of the HWHM. The standard deviations used by these authors to compute the damping envelope will thus range over a factor greater than two for the same value of alpha.

Less variation occurs in the gaussian spread-of-focus envelope. O'Keefe[1] and Spence[2] set the standard deviation equal to the spread of focus, delta; Reimer, Krivanek, and Stadelmann set delta equal to the FWHM of the gaussian. The figure shows the different damping envelopes obtained for the same nominal values of alpha and delta by the various expressions (the numbers on the curves indicate the references). This result illustrates how differences in TCCs, and thus simulated images, can arise.

[1]. M.A. O'Keefe, Ultramicroscopy 47 (1992) 282-297.

Jan-Olle Malm and Michael A. O'Keefe, Proceedings 51st MSA (1993) 974-975.

[2]. J.C.H. Spence, Experimental HREM, Oxford University Press, 1988.

L. Reimer, Transmission Electron Microscopy, Springer-Verlag, 1989.

O.L. Krivanek, HRTEM and Associated Techniques, Oxford University Press, Chapt 12, 1992.

[3]. P.A. Stadelmann, Ultramicroscopy21 (1987) 131. 
Main passbands are affected little by convergence

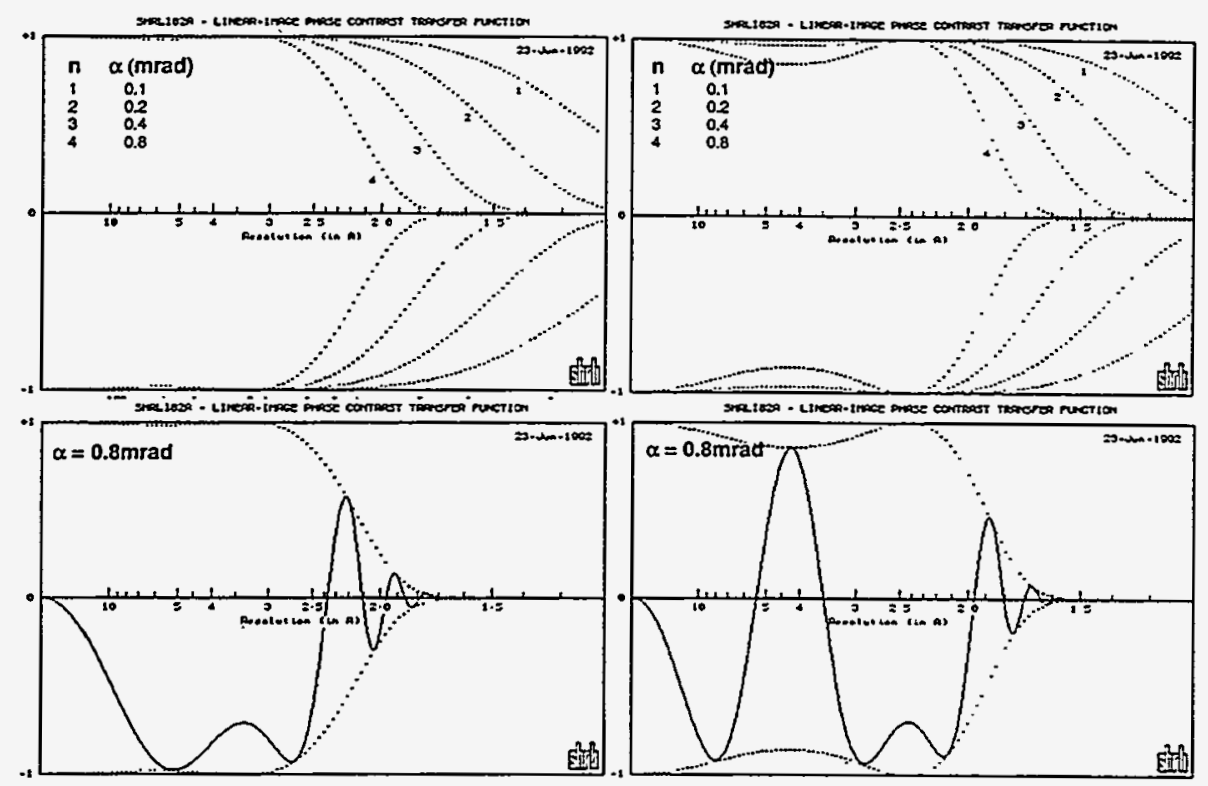

JEOL $200 \mathrm{CX}$ with spherical aberration coefficient of $1.2 \mathrm{~mm}$ at $200 \mathrm{keV}$

\section{CONVERGENCE ENVELOPES}

- Increasing convergence results in increased damping. The top plots are for $\alpha$ values of $0.1,0.2,0.4$, and 0.8 milliradian.

- Note that the shape of the damping function changes on going from Scherzer focus to second-passband defocus (compare the top plots).

- This shape change means that the main passband at each defocus is affected very little by convergence. 
Main passbands are affected more by spread-of-focus

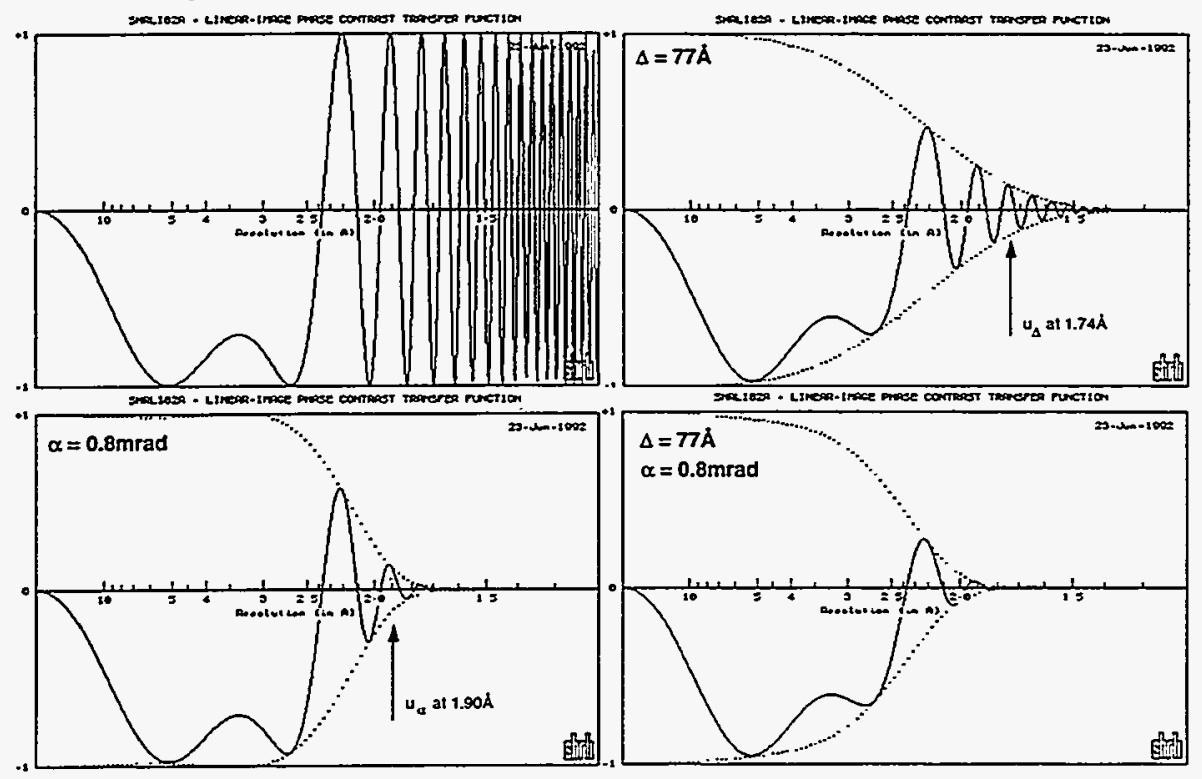

JEOL $200 \mathrm{CX}$ with spherical aberration coefficient of $1.2 \mathrm{~mm}$ at $200 \mathrm{keV}$

- MSA Tutorial III, 1994 Mchrel A. O'Keefe - 1 B

- In a microscope with a thermionic electron source (LaB6), the damping from spread-of-focus (top right) has more effect on the main passband than does the damping from convergence (bottom left).

- This is because the slope of the spread-of-focus envelope (top right) is more gradual than that of the convergence (bottom left).

- Thus the spread of focus has more effect on the passband, and the convergence has more on the high-frequency oscillations.

- The combined effect (bottom right) is to damp both passband and high-frequencies. 
Second passbands are affected strongly by spread-of-focus
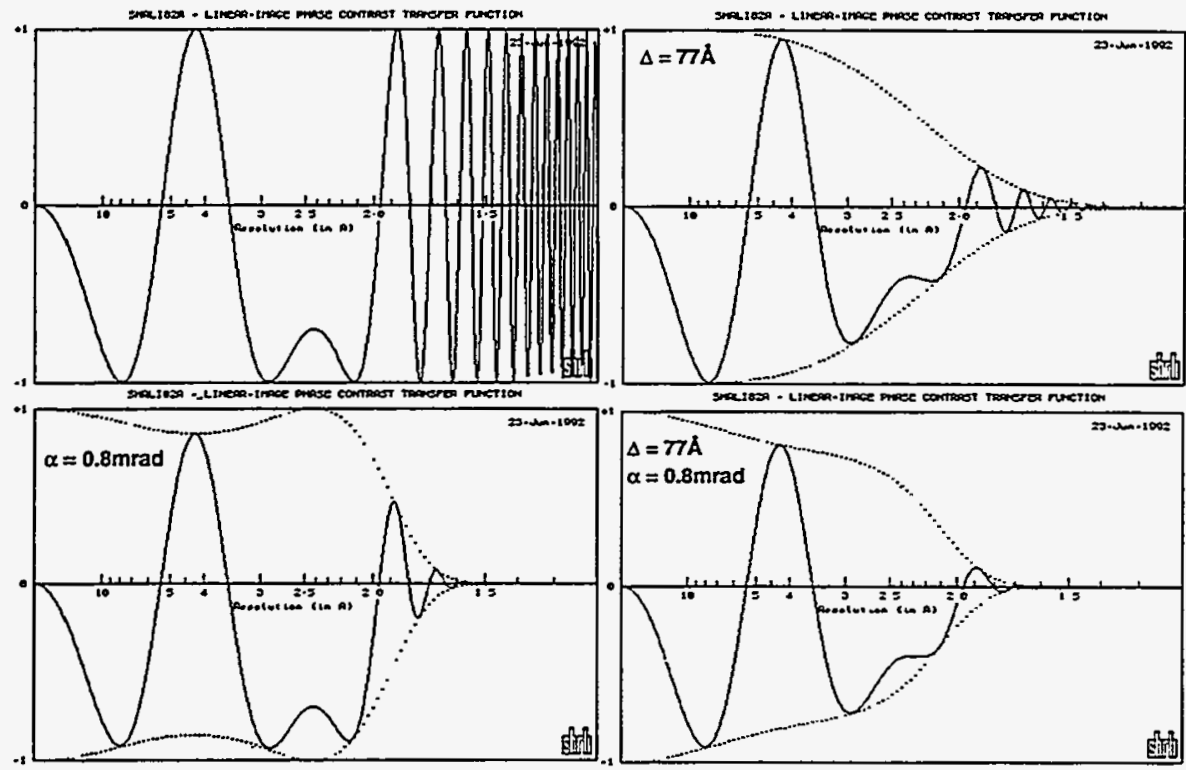

JEOL 200CX with spherical aberration coefficient of $1.2 \mathrm{~mm}$ at $200 \mathrm{keV}$

$\alpha$ and $\Delta$ envelopes at the second passband

- As we extend defocus to the second passband, we find that it is strongly damped by spread-of-focus (top right) compared with the effects of convergence (bottom left).

- Combined effect (bottom right) is to damp the hi-frequency oscillations almost entirely, and to attenuate the hi-frequency side of the main passband to less than $50 \%$ transfer between 2.5 and $2.0 \AA$. 


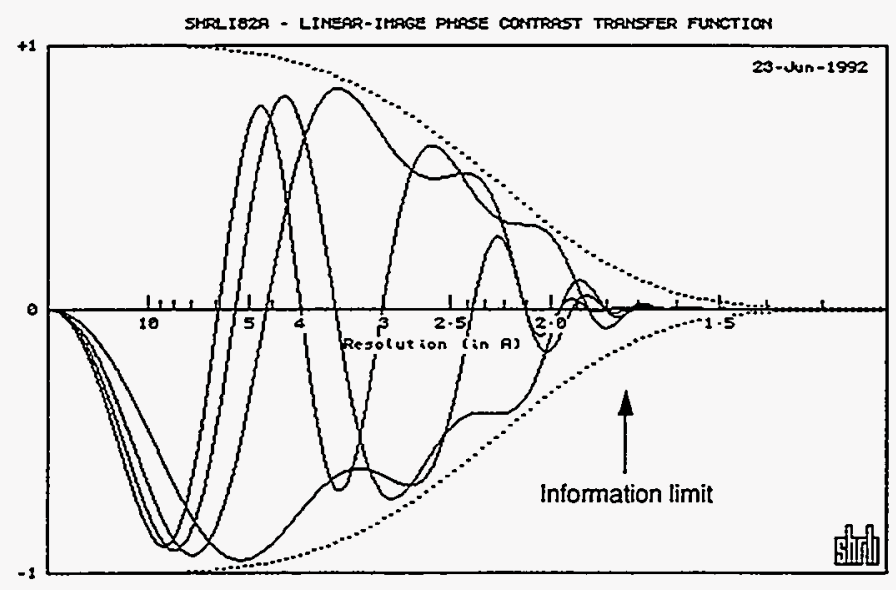

LaB6 focal series is limited to 4 or 5 passbands

\section{INFORMATION LIMIT - LaB6}

- Although we can use defocus series of images to retrieve information past the Scherzer resolution, the ultimate limit is set by the spread-offocus damping envelope. Note how this curve envelopes all the possible transfer functions.

- For this example with an LaB6 gun, the information limit (where the damping curve drops to $\exp \{-2\})$ is $1.74 \AA$.

- The plots show CTFs for the first four passbands, from the zeropassband (Scherzer) defocus (with no zero-crossings before the passband) through the third-passband defocus (with three zero-crossings). 
Focal series information is enveloped by spread-of-focus

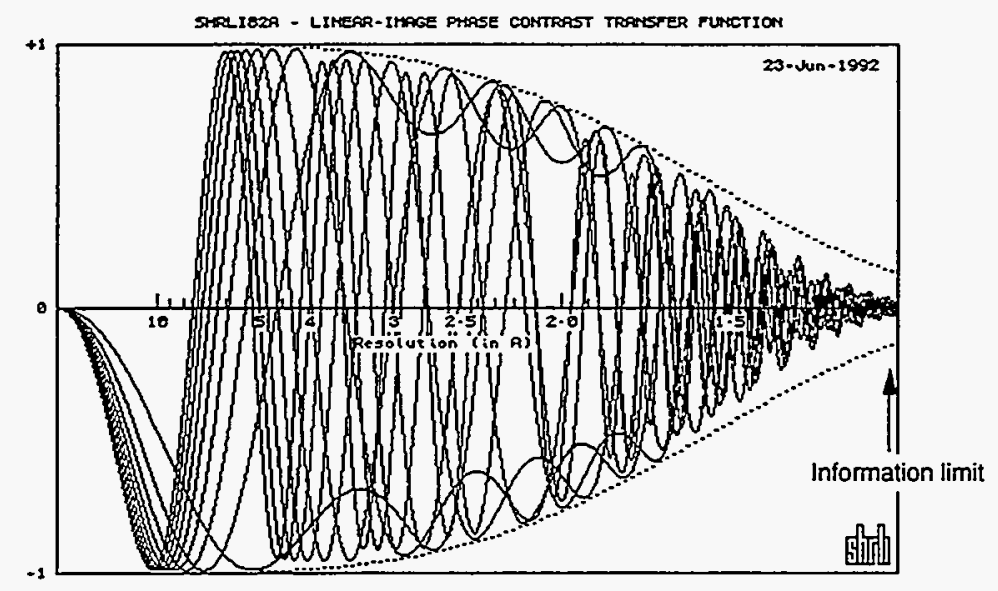

FEG focal series extends to 10 or more passbands

\section{INFORMATION LIMIT -- FEG}

- For this example with a FEG gun, the information limit (where the damping curve drops to $\exp (-2)$ ) is $1.22 \AA$. The right-hand edge of the plot is at $1.2 \AA$.

- Note the many useful CTFs. Each contains a main passband -although they can be difficult to see in this composite plot because of the oscillations from other defocus values. 
Scherzer Resolution:

The smallest spacing present in the "projected potential" or "structure" image - obtained at "optimum defocus" from a thin specimen.

Information Limit Resolution:

The smallest spacing transferred linearly into the image from the electron amplitude spectrum (the "diffraction pattern").

Fringe Resolution:

The smallest spacing present in the image, including non-linear transfers ("g minus g" interferences) from the amplitude spectrum into the intensity.

- MSA Tutorial III, 1994 Michael A. O'Keefe $-1 \mathbf{L}$

\section{RESOLUTION}

- There are three kind of "resolution" that can be defined for the HRTEM. All are specified in terms of the transfer of spatial information from the specimen into the image.

- Scherzer resolution -- all spacings down to the resolution limit are transferred with the same phase, generating a faithful "structure" image.

- Information Limit -- the smallest spacing (highest spatial frequency) that can be transferred linearly into the image, i.e. by interference with the central beam. Since some spacings will be misphased with respect to others in the image, such images are not "intuitively interpretable" and require comparison with simulations or image processing to correct phases.

- Fringe Resolution -- the smallest spacing in the image, wherever it came from. Usually generated by a non-linear, or "cross aperture" interference. 
Scherzer Resolution:

$$
d_{S}=0.67 C_{S}^{1 / 4} \lambda^{3 / 4}
$$

Information Limit Resolution:

$$
\mathrm{d}_{\mathrm{L}}=1 /\left.\mathrm{k}\right|_{\Delta}=\sqrt{\pi \lambda \Delta / 2}
$$

where

$$
\Delta=\mathrm{C}_{\mathrm{C}} \sqrt{\left(\frac{\delta \mathrm{V}}{\mathrm{V}}\right)^{2}+\left(\frac{2 \delta \mathrm{II}}{\mathrm{I}}\right)^{2}+\left(\frac{\delta \mathrm{E}}{\mathrm{E}}\right)^{2}}
$$

Fringe Image Resolution:

$$
\begin{gathered}
\mathrm{d}_{\mathrm{F}}=0.5 /\left.\mathbf{k}\right|_{\alpha} \quad|\mathbf{k}|_{\alpha}=S_{+}^{1 / 3}+S_{-}^{1 / 3} \\
\text { where } S_{ \pm}=\left[\frac{3.3}{4 \pi \alpha} \pm \sqrt{\frac{\varepsilon^{3}}{27 C_{S} \lambda^{2}}+\left(\frac{3.3}{4 \pi \alpha}\right)^{2}}\right] / C_{S} \lambda^{2} \\
\text { - MSA Tutorial II, } 1994
\end{gathered}
$$

\section{RESOLUTION EXPRESSIONS}

- We can write down expressions that quantify the resolutions. It turns out that the three resolutions are dependent on different microscope parameters.

- Scherzer resolution -- depends on $\mathbf{C}_{\mathbf{s}}$ and $\lambda$. At a given electron energy, $\mathrm{C}_{\mathrm{S}}$ determines the resolution.

- Information Limit -- depends on $\lambda$ and $\Delta$ (the spread of focus). At a given electron energy, $\Delta$ determines the resolution.

- Fringe Resolution -- depends upon $\alpha$, the convergence of the incident electron beam. Also on the defocus. And on $\mathrm{C}_{\mathrm{S}}$ and $\lambda$. Because information conveyed by second-order interferences can be very difficult to interpret, we generally avoid these interferences by working with thin crystals. 


\section{- Outline -}

What use is HRTEM image simulation?

History - image simulation was conceived for structure determination.

Uses -- the uses of image simulation and image processing -- structure determination is only one of five uses for image simulation.

Structure determination - recipes and examples of structure determination by matching of experimental images and simulated ones.

How does HRTEM image simulation work?

WPO -- interpreting images with the weak phase-object approximation; Why do some images "look like" the structure?

Beyond WPO -- the steps involved in image simulation; modelling the high-resolution TEM in the computer; computing dynamical diffraction; computing lens effects.

Using HRTEM image simulation

Bunning simulations -- obtaining values of simulation parameters.

- Handling defects -- what additional precautions are necessary?

\section{Using it -}

- Defects. Since image simulation algorithms require a periodic real space, how do we handle non-periodic defects?

- What extra problems arise? 


\section{Defect image simulation requires a large}

defect "unit cell"
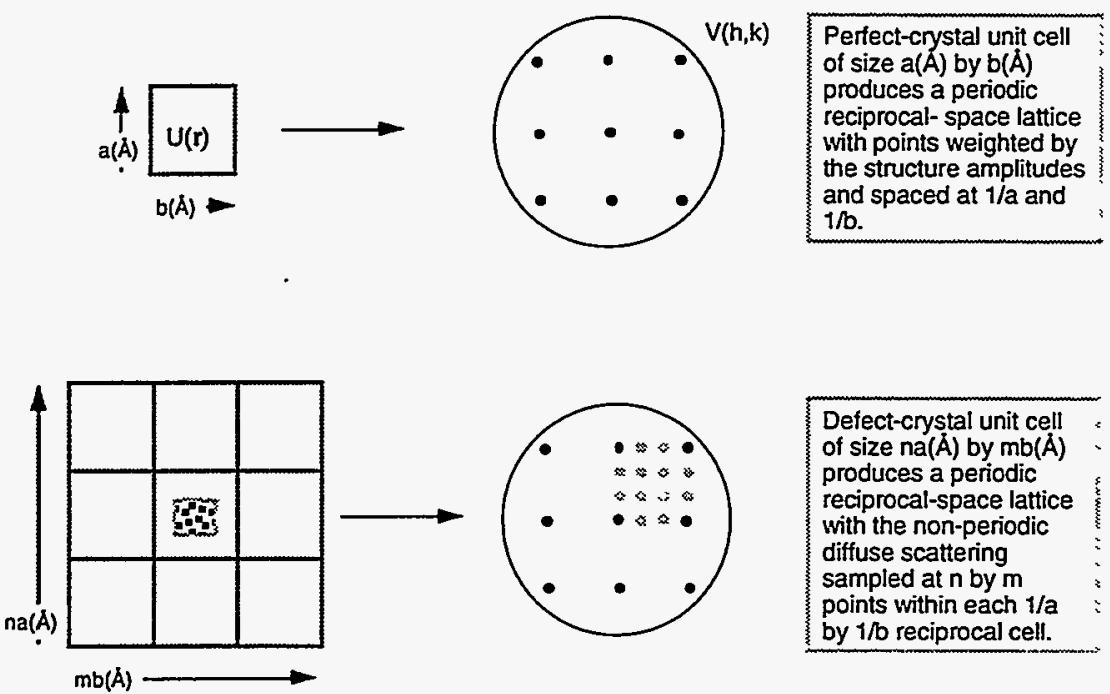

Defect-crystal unit cell of size na $(\bar{A})$ by $\mathrm{mb}(\bar{A})$ produces a periodic reciprocal-space lattice with the non-periodic diffuse scattering sampled at $\mathrm{n}$ by $\mathrm{m}$ points within each $1 / a$ by $1 / \mathrm{b}$ reciprocal cell.

- The non-periodic nature of a defect produces a (continuous) diffuse scattering in reciprocal space.

- Modeling the defect embedded in an area of $\mathbf{n}$ by $\mathbf{m}$ (perfect crystal) unit cells produces a sampling of the diffuse on a reciprocal-space lattice with $\mathbf{n}$ by $\mathbf{m}$ sampling within each (perfect-crystal) reciprocal unit cell.

- Increasing the values of $\mathbf{n}$ and $\mathbf{m}$ increases the sampling in reciprocal space and improves the definition of the diffuse scattering (but makes the calculation that much bigger). 


\section{Electron wave propagation within the specimen}

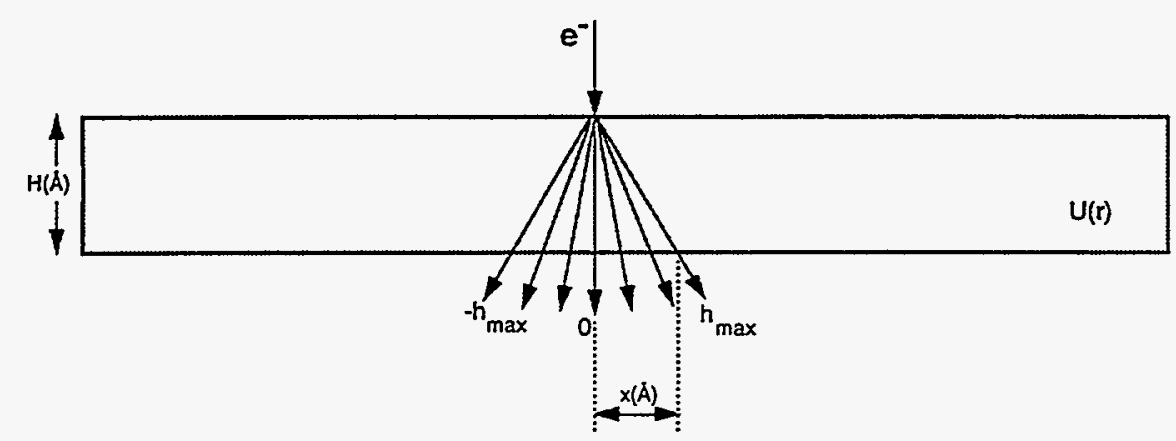

$$
\begin{aligned}
& \text { - } x(A) \text { is the sideways propagation of the highest-order diffracted beam considered in the } \\
& \text { multislice calculation: } \\
& \qquad x(\AA)=2 \theta \cdot H(A)=H \lambda / d_{\max }=H \lambda g_{\max }
\end{aligned}
$$

where $\lambda$ is the electron wavelength and $g_{\max }$ is the maximum scattering angle included.

- at the specimen exit surface, electrons $x(A)$ from an isolated defect will carry information about it

- information about a defect is carried by electrons at the specimen exit surface, up to a distance of $x(\AA)$ sideways from the position of the defect.

- where $x(\AA)$ is the sideways propagation of the highest-order diffracted beam considered in the multislice calculation:

$$
x(\AA)=2 \theta_{\max } \cdot H(\AA)
$$

- where $2 \theta_{\max }$ is given by the Bragg equation

$$
\mathrm{n} \lambda=2 \mathrm{~d} . \sin \theta
$$

or

$$
2 \sin \theta=n \lambda / \mathrm{d}=\lambda / \mathrm{d}_{\max }=\lambda \mathrm{g}_{\max }
$$

where $\lambda$ is the electron wavelength and $g_{\max }$ is the maximum scattering angle included in the multislice and $g_{\max }$ is the corresponding resolution. 
Electron wave propagation near defects

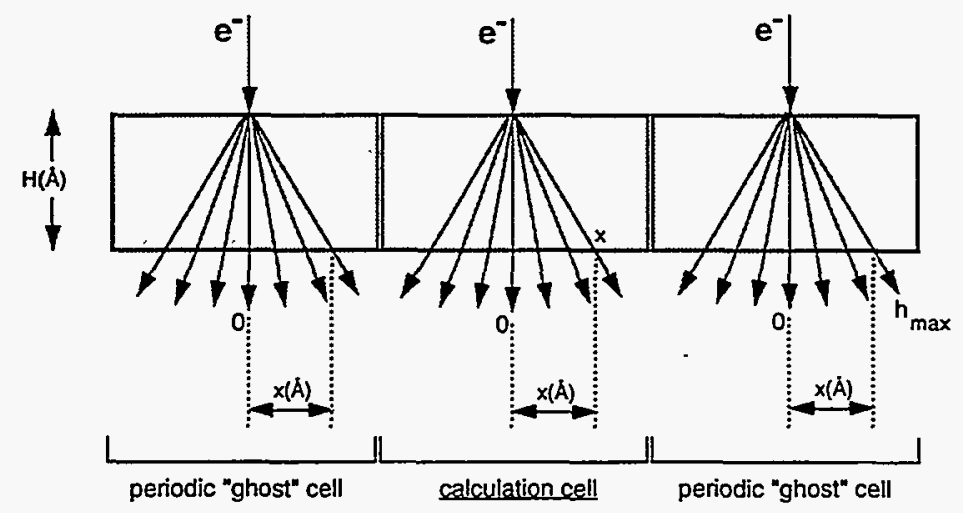

- the scattering calculation is periodic in two dimensions

- at the specimen exit surface, electrons $x(A)$ from the defect carry information about it

- at position $x$ the electron wavefield carries defect information

- Since the simulation procedure imposes a periodic nature upon the real-space structure used as the model, it is (strictly) impossible to simulate the image of an isolated defect.

- Thus to simulate defect images, we create a "supercell" of perfect crystal containing the defect. Then we are really simulating a periodic array of such defect cells.

- The supercell (or defect cell) must be large enough to ensure that the image of the periodic defect appears to be just like the image of an isolated one. 


\section{Short "defect cells" lead to scattering overlap}

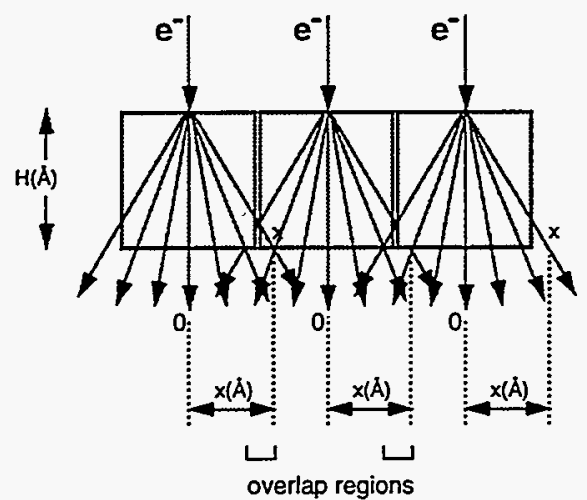

- the scattering calculation is periodic in two dimensions

- at the specimen exit surface, electrons $x\left(\AA^{\prime}\right)$ from the defect carry information about it

- if the defect cell has an extent of less than twice $x(\mathcal{A})$, the electron wavefield will carry incorrect information about the isolated defect in the overlap regions.

- If the defect cell is chosen to be too small, then the simulated image may contain contributions from the adjacent (but not seen) defect cell. These contibutions may contribute sufficiently to change the image within the cell under study. 


\section{Non-periodic "defect cells" have an additional}

"ghost" defect at the cell edge

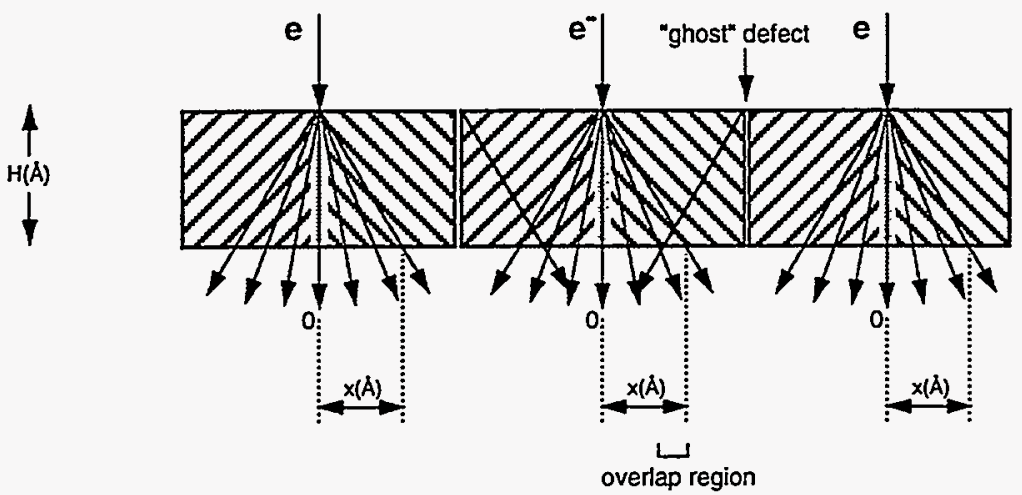

- non-periodically-continued defect cells introduce an additional defect at the cell edge

- at the specimen exit surface, electrons $x(\dot{A})$ from the defect carry information about it

- information from the "ghost" defect at the cell edge can spoil information from the defect under study

- Even if the defect cell is chosen large enough to avoid overlap problems with the same defect in the adjacent defect cell, contributions may occur from "ghost" defects present at the non-periodic boundary. 


\section{Even large non-periodic "defect cells" produce an image with a "discard" region at the cell edge}

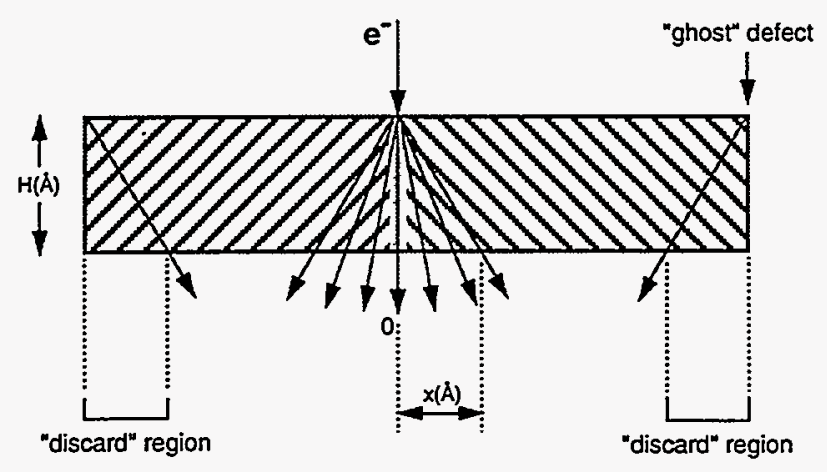

- non-periodically-continued defect cells introduce an additional defect at the cell edge

- at the specimen exit surface, electrons $x(\mathcal{A})$ from the defect carry information about it

- information from the "ghost" defect at the cell edge creates a region of image that should be discarded

- Even when the defect cell is chosen large enough to avoid overlap problems with boundary defects, contributions from boundary defects may still produce a distorted region of image close to these boundaries; this region should be discarded in order to avoid contributions from these non-physical "ghost" defects at the boundaries. 


\section{Defect cell for a $\Sigma 99$ grain boundary in aluminum}

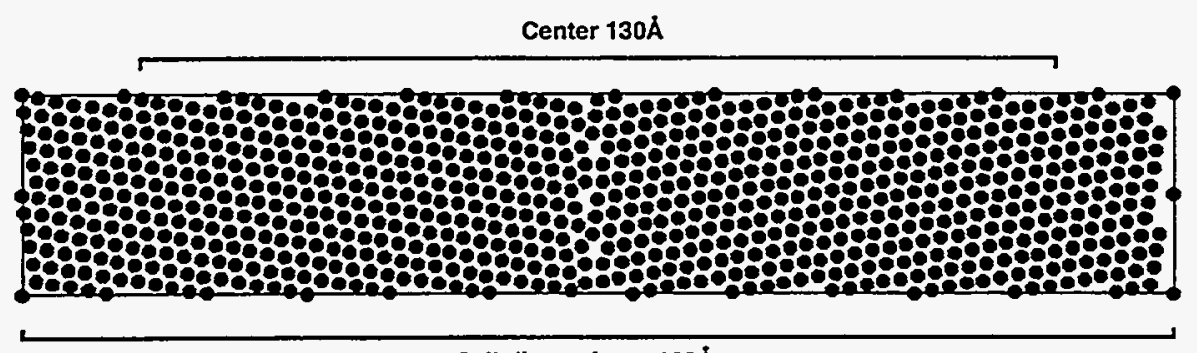

Cell dimension $=163 \AA ̊$

- Defect cell has dimensions of $28.42 \AA$ in the periodic direction (along the boundary) and $162.81 A$ in the non-periodic direction.

- Non-physical "boundary" at the unit-cell edges means that the nonperiodic defects are really spaced only $81.4 \AA$ apart.

- Center $130 \AA$ of image should be immune from any overlap effect from the non-periodic boundaries (at least for crystals no more than about $100 \AA$ thick and no further from Scherzer defocus than $1000 \AA)$.

This is a defect cell used in a project to determine the structure of a $\Sigma 99$ grain boundary in aluminum.

The defect cell is contructed (and viewed) in the [110] direction of the original perfect-crystal cell.

The structure is periodic along the grain boundary, so no overlap problems arise in this direction.

Perpendicular to the grain boundary, the structure is non-periodic, and the mandatory periodic-continuation imposed by the simulation procedure will create a non-physical "grain boundary" at the unit cell edge.

The non-periodic edge may produce spurious image detail in the regions adjacent to these edges, so it is wise to designate a "discard" region of $10 \AA$ to $15 \AA$ at each of these edges.

The defect cell is made large enough to avoid overlap problems (at least within the central $130 \AA$ ) in the direction perpendicular to the grain boundary. 


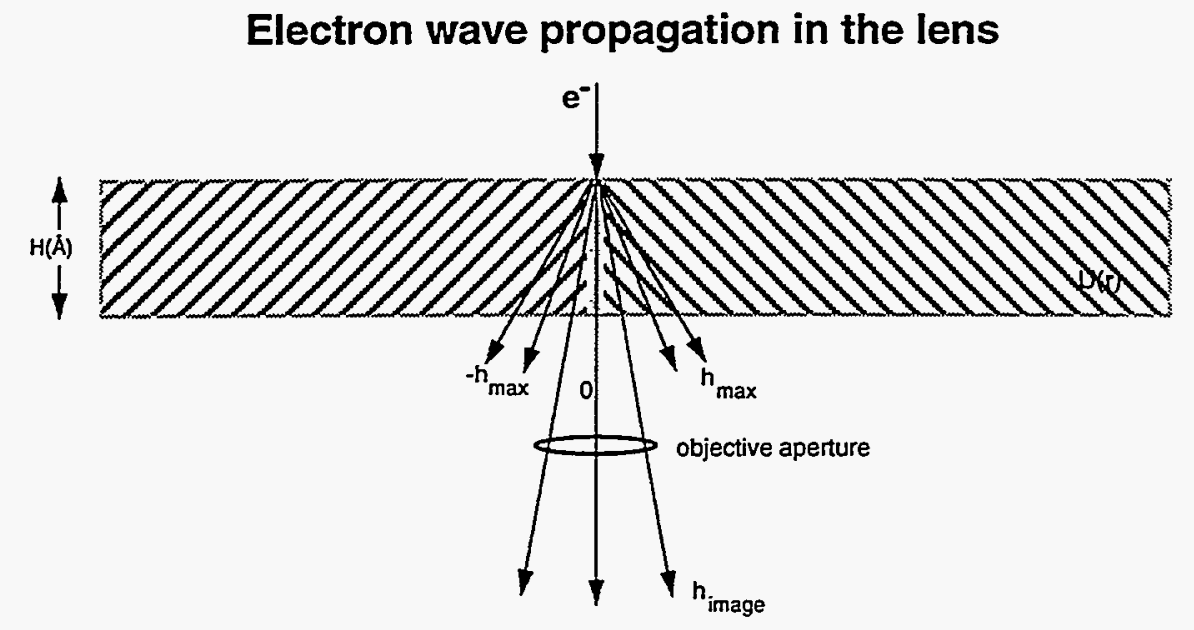

The sideways propagation of the highest-order diffracted beam that was included in the image calculation is $\varepsilon \lambda / d_{\text {irrage }}=\varepsilon \lambda g_{\text {image }}$

where -

$\lambda$ is the electron wavelength

$\varepsilon$ is the defocus from the Scherzer value

$g_{\text {image }}$ is the scattering angle of the highest-order beam included in the objective aperture.

- Just as sideways propagation of the diffracted beams within the specimen can lead to overlap problens, sideways propagation of the beams in the lens can also.

- In the specimen we deal with beams travelling through thicknesses of the order of $100 \AA$. In the lens the beams propagate through defocus distances of ten times this value, so the sideways effect has the potential to be ten times as great as for the specimen. However, the resolutions of the highest order beams contributing to the image are much less than those of the beams within the specimen (like $2 \AA$ instead of $0.5 \AA$ ), so the sideways displacements work out to be similar.

- Since Scherzer defocus gives the image that shows the closest approximation to a projection of the structure (potential), then it can be taken as the zero point for the measurement of sideways propagation due to defocus and focus-displacement can be measured from it. 


\section{Allowable Defect Separation}

Table of adjacent-defect interaction distances

Beam Wavelength Diffraction (100A thick) Image (1000A defocus)

\begin{tabular}{|c|c|c|c|c|c|}
\hline ( keV) & (A) & Besolution $(\dot{A})$ & Shift $(\dot{A})$ & Resolution(Å) & Shift \\
\hline 100 & 0.0370 & 0.50 & 15 & 4.0 & 20 \\
\hline 200 & 0.0251 & 0.33 & 15 & 2.0 & 25 \\
\hline 400 & 0.0164 & 0.25 & 13 & 1.5 & 22 \\
\hline 1000 & 0.0087 & 0.25 & 7 & 1.0 & 17 \\
\hline
\end{tabular}

Table shows typical minimum acceptable defect separation distances (wice the maximum distance over which "sideways" propagation of defect information will occur), in $\hat{A}$ per $100 \hat{A}$ of specimen thickness and $\hat{A}$ per $1000 \hat{A}$ of defocus, for several incident beam energies.

"Diffraction resolution" is the size of the "aperture" used in the diffraction calculation and determines the maximum scattering angle considered in the diffraction simulation.

Image resolution corresponds to the maximum scattering angle conisidered in the image simulation.

As a rule of thumb, keep defects $25-30 \hat{A}$ apart for every $100 \hat{A}$ thickness or $1000 \hat{A}$ defocus. And discard half this amount of non-periodically-continued cell edge.

\section{Constructing defect cells for image simulation}

Defect "unit cells" must be constructed such that adjacent defect images do not "interact" via the diffraction and imaging processes. The idea is to ensure that electrons that scatter from the defect do not propagate "sideways" by more than the distance to the cell boundary -- i.e. all defect-scattered electrons must remain within the local cell.

"Sideways" propagation arises in both the diffraction and imaging calculations. Within the specimen, the shift can be as large as the specimen thickness multiplied by the largest scattering angle considered in the diffraction calculation. Outside the specimen, the maximum shift is given by the defocus multiplied by (twice) the Bragg angle of the highestorder beam that contributes to the image.

The table shows that maximum shifts per $100 \AA$ specimen thickness are below $20 \AA$ for commonly-used computation parameters, and below $25 \AA$ per $1000 \AA$ defocus. The figures are maximum values because the higher-order beams representing them may be insufficiently strong to matter. 


\section{Defect Structure Determination by Image Simulation}

\section{Suggested Procedure:}

1. Image Maps -- Use image maps of the perfect structure to identify imaging conditions for defocus and thickness.

2. Experimental Focal Series -- Obtain focus series of experimental images if possible, else obtain best image as indicated by the map of defect-free images.

3. Simulated Defect - Simulate images from a model of the defect embedded in perfect matrix material, making the defect "unit cell" large enough to avoid overlap of adjacent defecis produced by "periodic continuation".

1. Structure determination of defects can be tedious because of the size of the defect cell that needs to be used, and because some defects require a minimum specimen thickness to survive. But much of the work can be carried out on the small-unit-cell perfect matrix surrounding the defect. Image maps of defocus and thickness can point the way to the best imaging conditions.

2. Just as for image matching of perfect crystal structures, matching of a defocus series is a more-stringent test of the model than is matching only a single image.

3. Ensure that the defect cell is large enough to avoid overlap problems. 